\title{
Drift Time Measurement in the ATLAS Liquid Argon Electromagnetic Calorimeter using Cosmic Muons
}

\author{
The ATLAS Collaboration ${ }^{\star \star \star \star}$
}

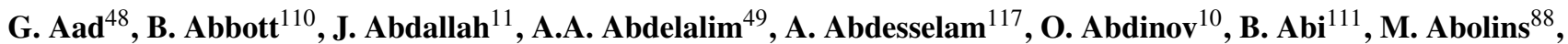
H. Abramowicz ${ }^{151}$, H. Abreu ${ }^{114}$, B.S. Acharya ${ }^{162 a, 162 b}$, D.L. Adams ${ }^{24}$, T.N. Addy ${ }^{56}$, J. Adelman ${ }^{173}$, C. Adorisio $^{36 a, 36 b}$, P. Adragna ${ }^{75}$, T. Adye ${ }^{128}$, S. Aefsky ${ }^{22}$, J.A. Aguilar-Saavedra ${ }^{123 a}$, M. Aharrouche ${ }^{81}$, S.P. Ahlen ${ }^{21}$, F. Ahles ${ }^{48}$, A. Ahmad ${ }^{146}$, H. Ahmed ${ }^{2}$, M. Ahsan ${ }^{40}$, G. Aielli132a,132b ${ }^{\text {, T. Akdogan }}{ }^{18}$, T.P.A. Åkesson ${ }^{79}$, G. Akimoto $^{153}$, A.V. Akimov ${ }^{94}$, A. Aktas $^{48}$, M.S. Alam ${ }^{1}$, M.A. Alam ${ }^{76}$, J. Albert ${ }^{167}$, S. Albrand ${ }^{55}$, M. Aleksa ${ }^{29}$, I.N. Aleksandrov ${ }^{65}$, F. Alessandria ${ }^{89 a}$, C. Alexa ${ }^{25 a}$, G. Alexander ${ }^{151}$, G. Alexandre ${ }^{49}$, T. Alexopoulos ${ }^{9}$, M. Alhroob ${ }^{20}$, M. Aliev $^{15}$, G. Alimonti ${ }^{89 a}$, J. Alison ${ }^{119}$, M. Aliyev ${ }^{10}$, P.P. Allport ${ }^{73}$, S.E. Allwood-Spiers ${ }^{53}$, J. Almond ${ }^{82}$, A. Aloisio ${ }^{102 a, 102 b}$, R. Alon ${ }^{169}$, A. Alonso ${ }^{79}$, M.G. Alviggi ${ }^{102 a, 102 b}$, K. Amako ${ }^{66}$, C. Amelung ${ }^{22}$, V.V. Ammosov ${ }^{127}$, A. Amorim ${ }^{123 b}$, G. Amorós ${ }^{165}$, N. Amram ${ }^{151}$, C. Anastopoulos ${ }^{138}$, T. Andeen ${ }^{29}$, C.F. Anders ${ }^{48}$, K.J. Anderson ${ }^{30}$, A. Andreazza ${ }^{89 a}, 89 \mathrm{~b}$, V. Andrei ${ }^{58 a}$, X.S. Anduaga ${ }^{70}$, A. Angerami ${ }^{34}$, F. Anghinolfi ${ }^{29}$, N. Anjos ${ }^{123 b}$, A. Antonaki ${ }^{8}$, M. Antonelli ${ }^{47}$,

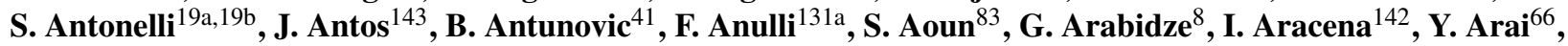
A.T.H. Arce $^{14}$, J.P. Archambault ${ }^{28}$, S. Arfaoui ${ }^{29, \text { a }}$, J.-F. Arguin ${ }^{14}$, T. Argyropoulos $^{9}$, E. Arik ${ }^{18, *}$, M. Arik ${ }^{18}$, A.J. Armbruster ${ }^{87}$, O. Arnaez ${ }^{4}$, C. Arnault ${ }^{114}$, A. Artamonov ${ }^{95}$, D. Arutinov ${ }^{20}$, M. Asai $^{142}$, S. Asai $^{153}$, R. Asfandiyarov ${ }^{170}$, S. Ask ${ }^{82}$, B. Åsman ${ }^{144}$, D. Asner ${ }^{28}$, L. Asquith ${ }^{77}$, K. Assamagan ${ }^{24}$, A. Astbury ${ }^{167}$, A. Astvatsatourov ${ }^{52}$, G. Atoian ${ }^{173}$, B. Auerbach ${ }^{173}$, E. Auge ${ }^{114}$, K. Augsten ${ }^{126}$, M. Aurousseau ${ }^{4}$, N. Austin ${ }^{73}$, G. Avolio ${ }^{161}$, R. Avramidou9 ${ }^{9}$ D. Axen ${ }^{166}$, C. Ay ${ }^{54}$, G. Azuelos ${ }^{93, b}$, Y. Azuma ${ }^{153}$, M.A. Baak ${ }^{29}$, C. Bacci $^{133 a, 133 b}$, A. Bach ${ }^{14}$, H. Bachacou ${ }^{135}$, K. Bachas ${ }^{29}$, M. Backes ${ }^{49}$, E. Badescu ${ }^{25 a}$, P. Bagnaia ${ }^{131 a, 131 b}$, Y. Bai ${ }^{32}$, D.C. Bailey ${ }^{156}$, T. Bain ${ }^{156}$, J.T. Baines ${ }^{128}$, O.K. Baker ${ }^{173}$, M.D. Baker ${ }^{24}$, S. Baker ${ }^{77}$, F. Baltasar Dos Santos Pedrosa ${ }^{29}$, E. Banas ${ }^{38}$,

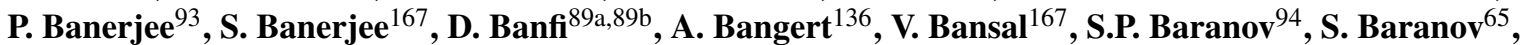
A. Barashkou ${ }^{65}$, T. Barber ${ }^{27}$, E.L. Barberio ${ }^{86}$, D. Barberis ${ }^{50 a, 50 b}$, M. Barbero ${ }^{20}$, D.Y. Bardin ${ }^{65}$, T. Barillari ${ }^{99}$, M. Barisonzi ${ }^{172}$, T. Barklow ${ }^{142}$, N. Barlow ${ }^{27}$, B.M. Barnett ${ }^{128}$, R.M. Barnett ${ }^{14}$, S. Baron ${ }^{29}$, A. Baroncelli $^{133 a}$, A.J. Barr ${ }^{117}$, F. Barreiro ${ }^{80}$, J. Barreiro Guimarães da Costa ${ }^{57}$, P. Barrillon ${ }^{114}$, N. Barros ${ }^{123 b}$, R. Bartoldus ${ }^{142}$, D. Bartsch ${ }^{20}$, J. Bastos ${ }^{123 b}$, R.L. Bates ${ }^{53}$, L. Batkova ${ }^{143}$, J.R. Batley ${ }^{27}$, A. Battaglia ${ }^{16}$, M. Battistin ${ }^{29}$, F. Bauer $^{135}$,

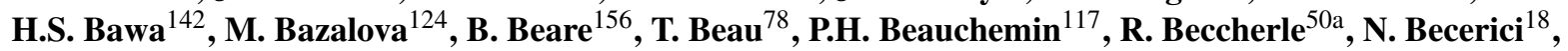
P. Bechtle ${ }^{41}$, G.A. Beck ${ }^{75}$, H.P. Beck ${ }^{16}$, M. Beckingham ${ }^{48}$, K.H. Becks ${ }^{172}$, I. Bedajanek ${ }^{126}$, A.J. Beddall ${ }^{18, c}$,

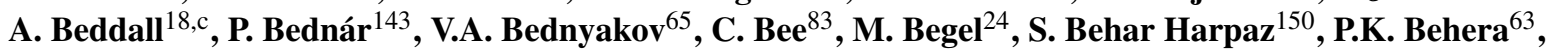
M. Beimforde ${ }^{99}$, C. Belanger-Champagne ${ }^{164}$, P.J. Bell ${ }^{82}$, W.H. Bell ${ }^{49}$, G. Bella ${ }^{151}$, L. Bellagamba ${ }^{19 a}$, F. Bellina ${ }^{29}$, M. Bellomo ${ }^{118 a}$, A. Belloni ${ }^{57}$, K. Belotskiy ${ }^{96}$, O. Beltramello ${ }^{29}$, S.Ben Ami ${ }^{150}$, O. Benary ${ }^{151}$, D. Benchekroun ${ }^{134 a}$,

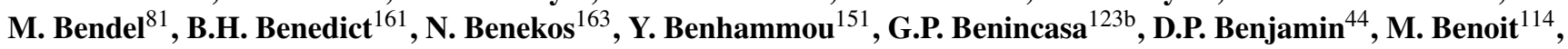
J.R. Bensinger ${ }^{22}$, K. Benslama ${ }^{129}$, S. Bentvelsen ${ }^{105}$, M. Beretta ${ }^{47}$, D. Berge ${ }^{29}$, E. Bergeaas Kuutmann ${ }^{144}$, N. Berger ${ }^{4}$, F. Berghaus ${ }^{167}$, E. Berglund ${ }^{49}$, J. Beringer ${ }^{14}$, K. Bernardet ${ }^{83}$, P. Bernat ${ }^{114}$, R. Bernhard ${ }^{48}$, C. Bernius ${ }^{77}$, T. Berry ${ }^{76}$, A. Bertin ${ }^{19 a, 19 b}$, M.I. Besana ${ }^{89 a, 89 b}$, N. Besson ${ }^{135}$, S. Bethke ${ }^{99}$, R.M. Bianchi ${ }^{48}$, M. Bianco ${ }^{72 a, 72 b}$, O. Biebel $^{98}$, J. Biesiada ${ }^{14}$, M. Biglietti ${ }^{131 a, 131 b}$, H. Bilokon ${ }^{47}$, M. Bindi ${ }^{19 a}, 19 b$, S. Binet $^{114}$, A. Bingul ${ }^{18, c}$, C. Bini $^{131 a, 131 b}$, C. Biscarat ${ }^{178}$, U. Bitenc ${ }^{48}$, K.M. Black ${ }^{57}$, R.E. Blair ${ }^{5}$, J.-B. Blanchard ${ }^{114}$, G. Blanchot ${ }^{29}$, C. Blocker $^{22}$, J. Blocki ${ }^{38}$, A. Blondel ${ }^{49}$, W. Blum ${ }^{81}$, U. Blumenschein ${ }^{54}$, G.J. Bobbink ${ }^{105}$, A. Bocci ${ }^{44}$, M. Boehler ${ }^{41}$, J. Boek ${ }^{172}$, N. Boelaert ${ }^{79}$, S. Böser ${ }^{77}$, J.A. Bogaerts ${ }^{29}$, A. Bogouch ${ }^{90, *}$, C. Bohm ${ }^{144}$, J. Bohm ${ }^{124}$, V. Boisvert ${ }^{76}$, T. Bold ${ }^{161, d}$, V. Boldea ${ }^{25 a}$, A. Boldyrev ${ }^{97}$, V.G. Bondarenko ${ }^{96}$, M. Bondioli ${ }^{161}$, M. Boonekamp ${ }^{135}$, S. Bordoni ${ }^{78}$, C. Borer $^{16}$, A. Borisov $^{127}$, G. Borissov ${ }^{71}$, I. Borjanovic ${ }^{72 a}$, S. Borroni ${ }^{131 a, 131 b}$, K. Bos ${ }^{105}$, D. Boscherini ${ }^{19 a}$, M. Bosman ${ }^{11}$, M. Bosteels $^{29}$, H. Boterenbrood ${ }^{105}$, J. Bouchami ${ }^{93}$, J. Boudreau ${ }^{122}$, E.V. Bouhova-Thacker ${ }^{71}$, C. Boulahouache $^{122}$, C. Bourdarios ${ }^{114}$, J. Boyd ${ }^{29}$, I.R. Boyko ${ }^{65}$, I. Bozovic-Jelisavcic ${ }^{12 b}$, J. Bracinik ${ }^{17}$, A. Braem ${ }^{29}$, P. Branchini $^{133 a}$, G.W. Brandenburg ${ }^{57}$, A. Brandt ${ }^{7}$, G. Brandt ${ }^{41}$, O. Brandt ${ }^{54}$, U. Bratzler ${ }^{154}$, B. Brau ${ }^{84}$, J.E. Brau ${ }^{113}$, H.M. Braun ${ }^{172}$, B. Brelier ${ }^{156}$, J. Bremer ${ }^{29}$, R. Brenner ${ }^{164}$, S. Bressler ${ }^{150}$, D. Breton ${ }^{114}$, D. Britton ${ }^{53}$, F.M. Brochu ${ }^{27}$, I. Brock ${ }^{20}$, R. Brock ${ }^{88}$, T.J. Brodbeck ${ }^{71}$, E. Brodet ${ }^{151}$, F. Broggi ${ }^{89 a}$, C. Bromberg ${ }^{88}$, G. Brooijmans ${ }^{34}$, W.K. Brooks ${ }^{31 b}$, 
G. Brown ${ }^{82}$, E. Brubaker ${ }^{30}$, P.A. Bruckman de Renstrom ${ }^{38}$, D. Bruncko ${ }^{143}$, R. Bruneliere ${ }^{48}$, S. Brunet $^{41}$, A. Bruni ${ }^{19 a}$, G. Bruni ${ }^{19 a}$, M. Bruschi ${ }^{19 a}$, T. Buanes ${ }^{13}$, F. Bucci ${ }^{49}$, J. Buchanan ${ }^{117}$, P. Buchholz ${ }^{140}$, A.G. Buckley ${ }^{45, e}$, I.A. Budagov ${ }^{65}$, B. Budick ${ }^{107}$, V. Büscher ${ }^{81}$, L. Bugge ${ }^{116}$, O. Bulekov ${ }^{96}$, M. Bunse ${ }^{42}$, T. Buran ${ }^{116}$, H. Burckhart ${ }^{29}$,

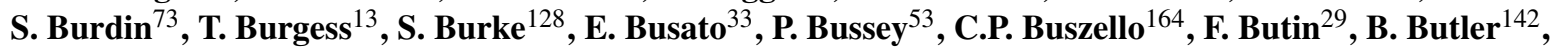
J.M. Butler ${ }^{21}$, C.M. Buttar ${ }^{53}$, J.M. Butterworth ${ }^{77}$, T. Byatt ${ }^{77}$, J. Caballero ${ }^{24}$, S. Cabrera Urbán ${ }^{165}$, D. Caforio ${ }^{19 a, 19 b}$, O. Cakir $^{3}$, P. Calafiura ${ }^{14}$, G. Calderini ${ }^{78}$, P. Calfayan ${ }^{98}$, R. Calkins ${ }^{5}$, L.P. Caloba ${ }^{23 a}$, R. Caloi ${ }^{131 a, 131 b}$, D. Calvet ${ }^{33}$, P. Camarri ${ }^{132 a, 132 b}$, M. Cambiaghi ${ }^{118 a}, 118 b$, D. Cameron ${ }^{116}$, F. Campabadal Segura ${ }^{165}$, S. Campana ${ }^{29}$, M. Campanelli ${ }^{77}$, V. Canale $^{102 a, 102 b}$, F. Canelli ${ }^{30}$, A. Canepa ${ }^{157 a}$, J. Cantero ${ }^{80}$, L. Capasso ${ }^{102 a, 102 b}$, M.D.M. Capeans

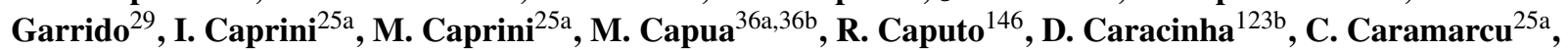
R. Cardarelli ${ }^{132 a}$, T. Carli ${ }^{29}$, G. Carlino ${ }^{102 a}$, L. Carminati ${ }^{89 a, 89 b}$, B. Caron ${ }^{2, b}$, S. Caron ${ }^{48}$, G.D. Carrillo Montoya ${ }^{170}$, S. Carron Montero ${ }^{156}$, A.A. Carter ${ }^{75}$, J.R. Carter $^{27}$, J. Carvalho ${ }^{123 b}$, D. Casadei ${ }^{107}$, M.P. Casado ${ }^{11}$, M. Cascella ${ }^{121 a, 121 b}$, C. Caso ${ }^{50,50 b, *}$, A.M. Castaneda Hernadez ${ }^{170}$, E. Castaneda-Miranda ${ }^{170}$, V. Castillo Gimenez $^{165}$, N. Castro ${ }^{123 a}$, G. Cataldi ${ }^{72 a}$, A. Catinaccio ${ }^{29}$, J.R. Catmore ${ }^{71}$, A. Cattai ${ }^{29}$, G. Cattani ${ }^{132 a, 132 b}$, S. Caughron ${ }^{34}$, D. Cauz ${ }^{162 a, 162 c}$, P. Cavalleri ${ }^{78}$, D. Cavalli ${ }^{89 a}$, M. Cavalli-Sforza ${ }^{11}$, V. Cavasinni ${ }^{121 a, 121 b}$, F. Ceradinii ${ }^{133 a, 133 b}$, A.S. Cerqueira ${ }^{23 a}$, A. Cerri ${ }^{29}$, L. Cerrito ${ }^{75}$, F. Cerutti ${ }^{47}$, S.A. Cetin ${ }^{18, f}$, F. Cevenini ${ }^{102 a, 102 b}$, A. Chafaq ${ }^{134 a}$, D. Chakraborty ${ }^{5}$, K. Chan $^{2}$, J.D. Chapman $^{27}$, J.W. Chapman ${ }^{87}$, E. Chareyre $^{78}$, D.G. Charlton ${ }^{17}$, V. Chavda ${ }^{82}$, S. Cheatham ${ }^{71}$, S. Chekanov ${ }^{5}$, S.V. Chekulaev ${ }^{157 a}$, G.A. Chelkov ${ }^{65}$, H. Chen $^{24}$, S. Chen ${ }^{32}$, T. Chen ${ }^{32}$, X. Chen ${ }^{170}$, S. Cheng ${ }^{32}$, A. Cheplakov ${ }^{65}$, V.F. Chepurnov ${ }^{65}$, R. Cherkaoui El Moursli ${ }^{134 d}$, V. Tcherniatine ${ }^{24}$, D. Chesneanu $^{25 a}$, E. Cheu ${ }^{6}$, S.L. Cheung ${ }^{156}$, L. Chevalier ${ }^{135}$, F. Chevallier ${ }^{135}$, V. Chiarella ${ }^{47}$, G. Chiefari ${ }^{102 a, 102 b}$, L. Chikovani $^{51}$, J.T. Childers ${ }^{58 a}$, A. Chilingarov ${ }^{71}$, G. Chiodini ${ }^{72 a}$, M. Chizhov ${ }^{65}$, G. Choudalakis $^{30}$, S. Chouridou ${ }^{136}$, I.A. Christidi ${ }^{152}$, A. Christov ${ }^{48}$, D. Chromek-Burckhart ${ }^{29}$, M.L. Chu ${ }^{149}$, J. Chudoba ${ }^{124}$,

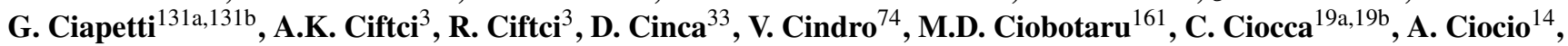
M. Cirilli ${ }^{87}$, M. Citterio ${ }^{89 a}$, A. Clark ${ }^{49}$, W. Cleland ${ }^{122}$, J.C. Clemens ${ }^{83}$, B. Clement ${ }^{55}$, C. Clement ${ }^{144}$, Y. Coadou $^{83}$, M. Cobal ${ }^{162 a, 162 \mathrm{c}}$, A. Coccaro ${ }^{50 \mathrm{a}, 50 \mathrm{~b}}$, J. Cochran ${ }^{64}$, S. Coelli ${ }^{89 a}$, J. Coggeshall ${ }^{163}$, E. Cogneras ${ }^{16}$, C.D. Cojocaru ${ }^{28}$, J. Colas ${ }^{4}$, B. Cole $^{34}$, A.P. Colijn ${ }^{105}$, C. Collard ${ }^{114}$, N.J. Collins ${ }^{17}$, C. Collins-Tooth ${ }^{53}$, J. Collot ${ }^{55}$, G. Colon ${ }^{84}$, P. Conde Muiño $^{123 b}$, E. Coniavitis ${ }^{164}$, M. Consonni ${ }^{104}$, S. Constantinescu ${ }^{25 a}$, C. Conta ${ }^{118 a, 118 b}$, F. Conventi ${ }^{102 a, g}$, J. Cook $^{29}$, M. Cooke ${ }^{34}$, B.D. Cooper ${ }^{75}$, A.M. Cooper-Sarkar ${ }^{117}$, N.J. Cooper-Smith ${ }^{76}$, K. Copic ${ }^{34}$, T. Cornelissen ${ }^{50 a, 50 b}$, M. Corradi ${ }^{19 a}$, F. Corriveau ${ }^{85, h}$, A. Corso-Radu ${ }^{161}$, A. Cortes-Gonzalez ${ }^{163}$, G. Cortiana ${ }^{99}$, G. Costa ${ }^{89 a}$, M.J. Costa ${ }^{165}$, D. Costanzo ${ }^{138}$, T. Costin ${ }^{30}$, D. Côté ${ }^{41}$, R. Coura Torres ${ }^{23 a}$, L. Courneyea ${ }^{167}$, G. Cowan ${ }^{76}$, C. Cowden ${ }^{27}$, B.E. Cox ${ }^{82}$, K. Cranmer ${ }^{107}$, J. Cranshaw $^{5}$, M. Cristinziani ${ }^{20}$, G. Crosetti ${ }^{36 a, 36 b}$, R. Crupi $^{72 a, 72 b}$, S. Crépé-Renaudin ${ }^{55}$, C. Cuenca Almenar ${ }^{173}$, T. Cuhadar Donszelmann ${ }^{138}$, M. Curatolo ${ }^{47}$, C.J. Curtis ${ }^{17}$,

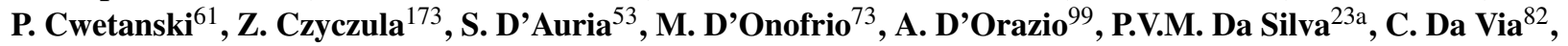
W. Dabrowski ${ }^{37}$, T. Dai ${ }^{87}$, C. Dallapiccola ${ }^{84}$, S.J. Dallison ${ }^{128, *}$, C.H. Daly ${ }^{137}$, M. Dam ${ }^{35}$, H.O. Danielsson ${ }^{29}$, D. Dannheim ${ }^{99}$, V. Dao ${ }^{49}$, G. Darbo ${ }^{50 a}$, G.L. Darlea ${ }^{25 a}$, W. Davey ${ }^{86}$, T. Davidek ${ }^{125}$, N. Davidson ${ }^{86}$, R. Davidson ${ }^{71}$, M. Davies ${ }^{93}$, A.R. Davison ${ }^{77}$, I. Dawson ${ }^{138}$, J.W. Dawson ${ }^{5}$, R.K. Daya ${ }^{39}$, K. De ${ }^{7}$, R. de Asmundis ${ }^{102 a}$, S. De Castro ${ }^{19 a, 19 b}$, P.E. De Castro Faria Salgado ${ }^{24}$, S. De Cecco ${ }^{78}$, J. de Graat ${ }^{98}$, N. De Groot ${ }^{104}$, P. de Jong ${ }^{105}$, E. De La Cruz-Burelo ${ }^{87}$, C. De La Taille ${ }^{114}$, L. De Mora ${ }^{71}$, M. De Oliveira Branco ${ }^{29}$, D. De Pedis ${ }^{131 a}$, A. De Salvo ${ }^{131 a}$, U. De Sanctis ${ }^{162 a, 162 c}$, A. De Santo ${ }^{147}$, J.B. De Vivie De Regie ${ }^{114}$, G. De Zorzi ${ }^{131 a, 131 b}$, S. Dean ${ }^{77}$, H. Deberg ${ }^{163}$, G. Dedes ${ }^{99}$, D.V. Dedovich ${ }^{65}$, P.O. Defay ${ }^{33}$, J. Degenhardt ${ }^{119}$, M. Dehchar ${ }^{117}$, C. Del Papa ${ }^{162 a, 162 c}$, J. Del Peso ${ }^{80}$, T. Del Prete ${ }^{121 a, 121 b}$, A. Dell'Acqua ${ }^{29}$, L. Dell'Asta ${ }^{89 a, 89 b}$, M. Della Pietra ${ }^{102 a, g}$, D. della Volpe $^{\text {102a,102b }}$, M. Delmastro ${ }^{29}$, N. Delruelle ${ }^{29}$, P.A. Delsart ${ }^{55}$, C. Deluca ${ }^{146}$, S. Demers ${ }^{173}$, M. Demichev ${ }^{65}$, B. Demirkoz ${ }^{11}$,

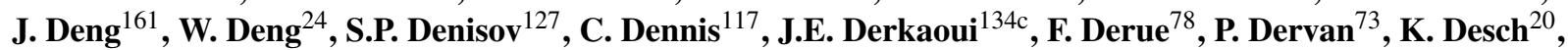
P.O. Deviveiros ${ }^{156}$, A. Dewhurst ${ }^{71}$, B. DeWilde ${ }^{146}$, S. Dhaliwal $^{156}$, R. Dhullipudi ${ }^{24, i}$, A. Di Ciaccio ${ }^{132 a, 132 b}$, L. Di Ciaccio ${ }^{4}$, A. Di Domenico ${ }^{131 a, 131 b}$, A. Di Girolamo ${ }^{29}$, B. Di Girolamo ${ }^{29}$, S. Di Luise ${ }^{133 a, 133 b}$, A. Di Mattia ${ }^{88}$, R. Di Nardo 132a,132b, A. Di Simone ${ }^{132 a, 132 b}$, R. Di Sipio ${ }^{19 a, 19 b}$, M.A. Diaz ${ }^{31 a}$, F. Diblen ${ }^{18}$, E.B. Diehl ${ }^{87}$, J. Dietrich ${ }^{48}$,

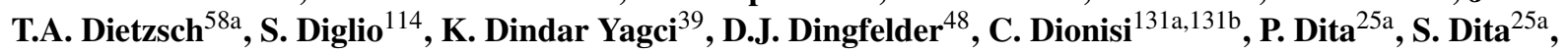
F. Dittus ${ }^{29}$, F. Djama ${ }^{83}$, R. Djilkibaev ${ }^{107}$, T. Djobava ${ }^{51}$, M.A.B. do Vale ${ }^{23 a}$, A. Do Valle Wemans ${ }^{123 b}$, T.K.O. Doan ${ }^{4}$, M. Dobbs ${ }^{85}$, D. Dobos ${ }^{29}$, E. Dobson ${ }^{29}$, M. Dobson ${ }^{161}$, J. Dodd ${ }^{34}$, T. Doherty ${ }^{53}$, Y. Doi ${ }^{66}$, J. Dolejsi ${ }^{125}$, I. Dolenc ${ }^{74}$, Z. Dolezal ${ }^{125}$, B.A. Dolgoshein ${ }^{96}$, T. Dohmae ${ }^{153}$, M. Donega ${ }^{119}$, J. Donini5 ${ }^{55}$, J. Dopke ${ }^{172}$, A. Doria ${ }^{102 a}$, A. Dos Anjos ${ }^{170}$, A. Dotti ${ }^{121 a, 121 b}$, M.T. Dova ${ }^{70}$, A. Doxiadis ${ }^{105}$, A.T. Doyle ${ }^{53}$, Z. Drasal $^{125}$, C. Driouichi' $^{35}$, M. Dris $^{9}$, J. Dubbert $^{99}$, E. Duchovni ${ }^{169}$, G. Duckeck ${ }^{98}$, A. Dudarev ${ }^{29}$, F. Dudziak ${ }^{114}$, M. Dührssen ${ }^{48}$, L. Duflot ${ }^{114}$, M.-A. Dufour ${ }^{85}$, M. Dunford ${ }^{30}$, A. Duperrin ${ }^{83}$, H.Duran Yildiz ${ }^{3, j}$, A. Dushkin ${ }^{22}$, R. Duxfield ${ }^{138}$, M. Dwuznik ${ }^{37}$, 
M. Düren ${ }^{52}$, W.L. Ebenstein ${ }^{44}$, J. Ebke ${ }^{98}$, S. Eckert ${ }^{48}$, S. Eckweiler ${ }^{81}$, K. Edmonds ${ }^{81}$, C.A. Edwards ${ }^{76}$, P. Eerola ${ }^{79, k}$, K. Egorov ${ }^{61}$, W. Ehrenfeld ${ }^{41}$, T. Ehrich ${ }^{99}$, T. Eifert $^{29}$, G. Eigen $^{13}$, K. Einsweiler ${ }^{14}$, E. Eisenhandler ${ }^{75}$, T. Ekelof ${ }^{164}$, M. El Kacimi ${ }^{4}$, M. Ellert ${ }^{164}$, S. Elles ${ }^{4}$, F. Ellinghaus ${ }^{81}$, K. Ellis ${ }^{75}$, N. Ellis ${ }^{29}$, J. Elmsheuser ${ }^{98}$, M. Elsing ${ }^{29}$, R. Ely ${ }^{14}$, D. Emeliyanov ${ }^{128}$, R. Engelmann ${ }^{146}$, A. Engl ${ }^{98}$, B. Epp ${ }^{62}$, A. Eppig ${ }^{87}$, V.S. Epshteyn ${ }^{95}$, A. Ereditato ${ }^{16}$, D. Eriksson ${ }^{144}$, I. Ermoline ${ }^{88}$, J. Ernst ${ }^{1}$, M. Ernst ${ }^{24}$, J. Ernwein ${ }^{135}$, D. Errede ${ }^{163}$, S. Errede $^{163}$, E. Ertel ${ }^{81}$, M. Escalier ${ }^{114}$, C. Escobar $^{165}$, X. Espinal Curull ${ }^{11}$, B. Esposito ${ }^{47}$, F. Etienne ${ }^{83}$, A.I. Etienvre ${ }^{135}$, E. Etzion ${ }^{151}$,

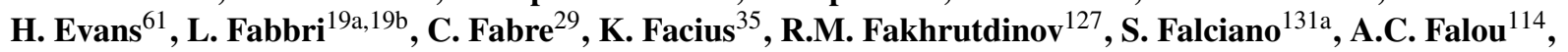
Y. Fang ${ }^{170}$, M. Fanti ${ }^{89 a, 89 b}$, A. Farbin ${ }^{7}$, A. Farilla ${ }^{133 a}$, J. Farley ${ }^{146}$, T. Farooque ${ }^{156}$, S.M. Farrington ${ }^{117}$, P. Farthouat ${ }^{29}$, F. Fassi ${ }^{165}$, P. Fassnacht ${ }^{29}$, D. Fassouliotis ${ }^{8}$, B. Fatholahzadeh ${ }^{156}$, L. Fayard ${ }^{14}$, F. Fayette ${ }^{54}$,

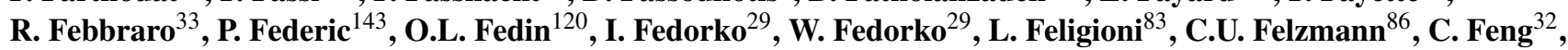
E.J. Feng ${ }^{30}$, A.B. Fenyuk ${ }^{127}$, J. Ferencei ${ }^{143}$, J. Ferland ${ }^{93}$, B. Fernandes ${ }^{123 b}$, W. Fernando ${ }^{108}$, S. Ferrag $^{53}$,

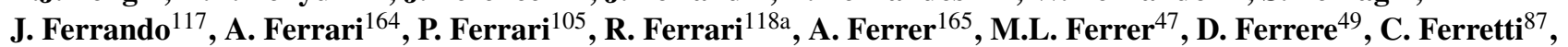
M. Fiascaris $^{117}$, F. Fiedler ${ }^{81}$, A. Filipčic ${ }^{74}$, A. Filippas ${ }^{9}$, F. Filthaut ${ }^{104}$, M. Fincke-Keeler ${ }^{167}$, M.C.N. Fiolhais ${ }^{123 b}$,

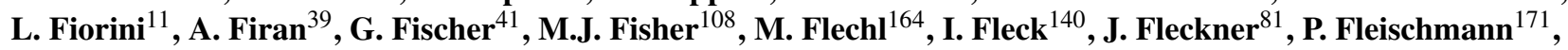
S. Fleischmann ${ }^{20}$, T. Flick ${ }^{172}$, L.R. Flores Castillo ${ }^{170}$, M.J. Flowerdew ${ }^{99}$, F. Föhlisch ${ }^{58 a}$, M. Fokitis ${ }^{9}$, T. Fonseca Martin ${ }^{76}$, D.A. Forbush ${ }^{137}$, A. Formica ${ }^{135}$, A. Forti ${ }^{82}$, D. Fortin ${ }^{157 a}$, J.M. Foster ${ }^{82}$, D. Fournier ${ }^{114}$, A. Foussat ${ }^{29}$, A.J. Fowler ${ }^{44}$, K. Fowler ${ }^{136}$, H. Fox ${ }^{71}$, P. Francavilla121a,121b, S. Franchino ${ }^{118 a, 118 b}$, D. Francis ${ }^{29}$, M. Franklin ${ }^{57}$, S. Franz ${ }^{29}$, M. Fraternali ${ }^{118 a, 118 b}$, S. Fratina ${ }^{119}$, J. Freestone ${ }^{82}$, S.T. French ${ }^{27}$, R. Froeschl ${ }^{29}$, D. Froidevaux ${ }^{29}$, J.A. Frost ${ }^{27}$, C. Fukunaga ${ }^{154}$, E. Fullana Torregrosa ${ }^{5}$, J. Fuster ${ }^{165}$, C. Gabaldon ${ }^{80}$, O. Gabizon ${ }^{169}$, T. Gadfort ${ }^{24}$, S. Gadomski ${ }^{49}$, G. Gagliardi ${ }^{50,50 b}$, P. Gagnon ${ }^{61}$, C. Galea ${ }^{98}$, E.J. Gallas ${ }^{117}$, M.V. Gallas ${ }^{29}$, V. Gallo ${ }^{16}$, B.J. Gallop ${ }^{128}$, P. Gallus ${ }^{124}$, E. Galyaev ${ }^{40}$, K.K. Gan ${ }^{108}$, Y.S. Gao ${ }^{142,1}$, A. Gaponenko ${ }^{14}$, M. Garcia-Sciveres ${ }^{14}$, C. García ${ }^{165}$, J.E. García Navarro ${ }^{49}$, R.W. Gardner ${ }^{30}$, N. Garelli ${ }^{29}$, H. Garitaonandia ${ }^{105}$, V. Garonne ${ }^{29}$, C. Gatti ${ }^{47}$, G. Gaudio $^{118 a}$, O. Gaumer ${ }^{49}$, P. Gauzzi ${ }^{131 a, 131 b}$, I.L. Gavrilenko ${ }^{94}$, C. Gay ${ }^{166}$, G. Gaycken ${ }^{20}$, J.-C. Gayde ${ }^{29}$, E.N. Gazis ${ }^{9}$, P. Ge ${ }^{32}$, C.N.P. Gee ${ }^{128}$, Ch. Geich-Gimbel ${ }^{20}$, K. Gellerstedt ${ }^{144}$, C. Gemme ${ }^{50 a}$, M.H. Genest ${ }^{98}$, S. Gentile ${ }^{131 \mathrm{a}, 131 \mathrm{~b}}$, F. Georgatos ${ }^{9}$, S. George ${ }^{76}$, P. Gerlach ${ }^{172}$, A. Gershon ${ }^{151}$, C. Geweniger $^{58 \mathrm{a}}$, H. Ghazlane ${ }^{134 \mathrm{~d}}$, P. Ghez ${ }^{4}$, N. Ghodbane ${ }^{33}$, B. Giacobbe ${ }^{19 a}$, S. Giagu ${ }^{131 a, 131 b}$, V. Giakoumopoulou ${ }^{8}$, V. Giangiobbe ${ }^{121 a, 121 b}$, F. Gianotti ${ }^{29}$, B. Gibbard ${ }^{24}$, A. Gibson ${ }^{156}$, S.M. Gibson ${ }^{117}$, L.M. Gilbert ${ }^{117}$, M. Gilchriese $^{14}$, V. Gilewsky ${ }^{91}$, A.R. Gillman ${ }^{128}$, D.M. Gingrich ${ }^{2, b}$, J. Ginzburg ${ }^{151}$, N. Giokaris ${ }^{8}$, M.P. Giordani ${ }^{162 a, 162 c}$, R. Giordano ${ }^{102 a, 102 b}$, P. Giovannini ${ }^{99}$, P.F. Giraud ${ }^{29}$, P. Girtler ${ }^{62}$, D. Giugni ${ }^{89 a}$, P. Giusti ${ }^{19 a}$, B.K. Gjelsten ${ }^{116}$, L.K. Gladilin ${ }^{97}$, C. Glasman $^{80}$, A. Glazov ${ }^{41}$, K.W. Glitza ${ }^{172}$, G.L. Glonti ${ }^{65}$, J. Godfrey ${ }^{141}$, J. Godlewski ${ }^{29}$, M. Goebel ${ }^{41}$, T. Göpfert ${ }^{43}$, C. Goeringer ${ }^{81}$, C. Gössling ${ }^{42}$, T. Göttfert ${ }^{99}$, V. Goggi ${ }^{118 a, 118 b, m}$, S. Goldfarb ${ }^{87}$, D. Goldin ${ }^{39}$, T. Golling ${ }^{173}$, N.P. Gollub ${ }^{29}$, A. Gomes ${ }^{123 b}$, L.S. Gomez Fajardo ${ }^{41}$, R. Gonçalo ${ }^{76}$, L. Gonella ${ }^{20}$, C. Gong ${ }^{32}$,

S. González de la Hoz ${ }^{165}$, M.L. Gonzalez Silva ${ }^{26}$, S. Gonzalez-Sevilla ${ }^{49}$, J.J. Goodson ${ }^{146}$, L. Goossens ${ }^{29}$, P.A. Gorbounov ${ }^{156}$, H.A. Gordon ${ }^{24}$, I. Gorelov ${ }^{103}$, G. Gorfine ${ }^{172}$, B. Gorini ${ }^{29}$, E. Gorini ${ }^{72 a, 72 b}$, A. Gorišek ${ }^{74}$, E. Gornicki ${ }^{38}$, V.N. Goryachev ${ }^{127}$, B. Gosdzik ${ }^{41}$, M. Gosselink ${ }^{105}$, M.I. Gostkin ${ }^{65}$, I. Gough Eschrich ${ }^{161}$, M. Gouighri ${ }^{134 a}$, D. Goujdami ${ }^{134 a}$, M.P. Goulette ${ }^{49}$, A.G. Goussiou ${ }^{137}$, C. Goy ${ }^{4}$, I. Grabowska-Bold ${ }^{161, d}$, P. Grafström ${ }^{29}$, K.-J. Grahn ${ }^{145}$, L. Granado Cardoso ${ }^{123 b}$, F. Grancagnolo ${ }^{72 a}$, S. Grancagnolo ${ }^{15}$, V. Grassi ${ }^{89 a}$, V. Gratchev ${ }^{120}$, N. Grau ${ }^{34}$, H.M. Gray ${ }^{34, n}$, J.A. Gray ${ }^{146}$, E. Graziani ${ }^{133 a}$, B. Green ${ }^{76}$, T. Greenshaw ${ }^{73}$, Z.D. Greenwood ${ }^{24, i}$, I.M. Gregor ${ }^{41}$, P. Grenier ${ }^{142}$, E. Griesmayer ${ }^{46}$, J. Griffiths ${ }^{137}$, N. Grigalashvili ${ }^{65}$, A.A. Grillo ${ }^{136}$, K. Grimm ${ }^{146}$, S. Grinstein ${ }^{11}$, Y.V. Grishkevich ${ }^{97}$, L.S. Groer ${ }^{156}$, J. Grognuz ${ }^{29}$, M. Groh ${ }^{99}$, M. Groll ${ }^{81}$, E. Gross ${ }^{169}$, J. Grosse-Knetter ${ }^{54}$, J. Groth-Jensen ${ }^{79}$, K. Grybel ${ }^{140}$, V.J. Guarino ${ }^{5}$, C. Guicheney ${ }^{33}$, A. Guida ${ }^{72 a, 72 b}$, T. Guillemin ${ }^{4}$,

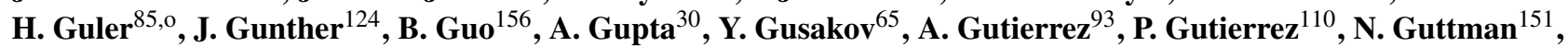

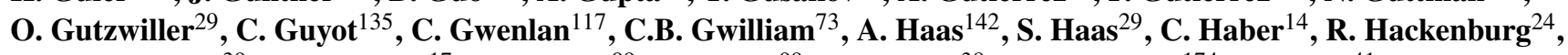
H.K. Hadavand ${ }^{39}$, D.R. Hadley ${ }^{17}$, P. Haefner ${ }^{99}$, R. Härtel ${ }^{99}$, Z. Hajduk ${ }^{38}$, H. Hakobyan ${ }^{174}$, J. Haller ${ }^{41, p}$,

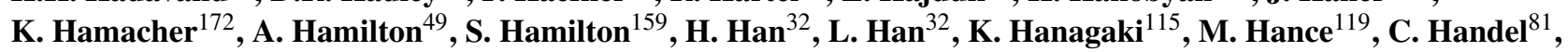

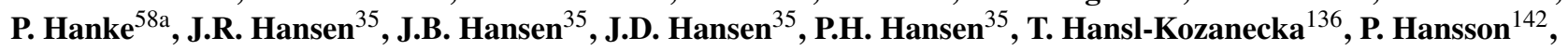

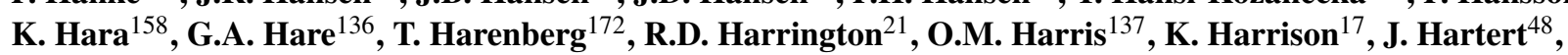

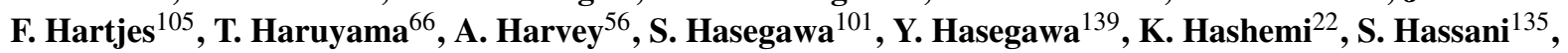
M. Hatch ${ }^{29}$, F. Haug ${ }^{29}$, S. Haug ${ }^{16}$, M. Hauschild ${ }^{29}$, R. Hauser ${ }^{88}$, M. Havranek ${ }^{124}$, C.M. Hawkes ${ }^{17}$, R.J. Hawkings ${ }^{29}$,

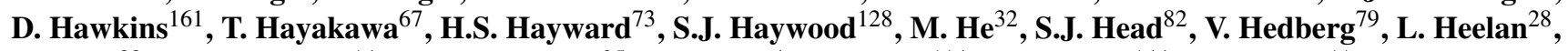
S. Heim ${ }^{88}$, B. Heinemann ${ }^{14}$, S. Heisterkamp ${ }^{35}$, L. Helary ${ }^{4}$, M. Heller ${ }^{114}$, S. Hellman ${ }^{144}$, C. Helsens ${ }^{11}$,

T. Hemperek $^{20}$, R.C.W. Henderson ${ }^{71}$, M. Henke ${ }^{58 a}$, A. Henrichs ${ }^{54}$, A.M. Henriques Correia ${ }^{29}$, S. Henrot-Versille ${ }^{114}$, 
C. Hensel $^{54}$, T. Henß ${ }^{172}$, Y. Hernández Jiménez ${ }^{165}$, A.D. Hershenhorn ${ }^{150}$, G. Herten ${ }^{48}$, R. Hertenberger ${ }^{98}$, L. Hervas ${ }^{29}$, N.P. Hessey ${ }^{105}$, A. Hidvegi ${ }^{144}$, E. Higón-Rodriguez ${ }^{165}$, D. Hill ${ }^{5}$, , J.C. Hill ${ }^{27}$, K.H. Hiller ${ }^{41}$, S. Hillert ${ }^{144}$, S.J. Hillier ${ }^{17}$, I. Hinchliffe ${ }^{14}$, E. Hines ${ }^{119}$, M. Hirose ${ }^{115}$, F. Hirsch ${ }^{42}$, D. Hirschbuehl ${ }^{172}$, J. Hobbs ${ }^{146}$, N. Hod ${ }^{151}$, M.C. Hodgkinson ${ }^{138}$, P. Hodgson ${ }^{138}$, A. Hoecker ${ }^{29}$, M.R. Hoeferkamp ${ }^{103}$, J. Hoffman ${ }^{39}$, D. Hoffmann ${ }^{83}$, M. Hohlfeld ${ }^{81}$, S.O. Holmgren ${ }^{144}$, T. Holy ${ }^{126}$, J.L. Holzbauer ${ }^{88}$, Y. Homma ${ }^{67}$, P. Homola ${ }^{126}$, T. Horazdovsky ${ }^{126}$, T. Hori ${ }^{67}$, C. Horn ${ }^{142}$, S. Horner ${ }^{48}$, S. Horvat ${ }^{99}$, J.-Y. Hostachy ${ }^{55}$, S. Hou ${ }^{149}$, M.A. Houlden ${ }^{73}$, A. Hoummada ${ }^{134 a}$, T. Howe ${ }^{39}$, J. Hrivnac ${ }^{114}$, T. Hryn'ova ${ }^{4}$, P.J. Hsu ${ }^{173}$, S.-C. Hsu ${ }^{14}$, G.S. Huang ${ }^{110}$, Z. Hubacek ${ }^{126}$, F. Hubaut ${ }^{83}$, F. Huegging ${ }^{20}$, E.W. Hughes ${ }^{34}$, G. Hughes ${ }^{71}$, R.E. Hughes-Jones ${ }^{82}$, P. Hurst ${ }^{57}$, M. Hurwitz ${ }^{30}$, U. Husemann ${ }^{41}$, N. Huseynov ${ }^{10}$, J. Huston ${ }^{88}$, J. Huth ${ }^{57}$, G. Iacobucci ${ }^{102 a}$, G. Iakovidis ${ }^{9}$, I. Ibragimov ${ }^{140}$, L. Iconomidou-Fayard ${ }^{114}$, J. Idarraga $^{157 b}$, P. Iengo ${ }^{4}$, O. Igonkina ${ }^{105}$, Y. Ikegami ${ }^{66}$, M. Ikeno ${ }^{66}$, Y. Ilchenko ${ }^{39}$, D. Iliadis $^{152}$, Y. Ilyushenka ${ }^{65}$, M. Imori ${ }^{153}$, T. Ince ${ }^{167}$, P. Ioannou ${ }^{8}$, M. Iodice ${ }^{133 a}$, A. Irles Quiles ${ }^{165}$, A. Ishikawa ${ }^{67}$, M. Ishino ${ }^{66}$,

R. Ishmukhametov ${ }^{39}$, T. Isobe ${ }^{153}$, V. Issakov ${ }^{173, *}$, C. Issever ${ }^{117}$, S. Istin ${ }^{18}$, Y. Itoh ${ }^{101}$, A.V. Ivashin ${ }^{127}$, H. Iwasaki ${ }^{66}$, J.M. Izen ${ }^{40}$, V. Izzo ${ }^{102 a}$, B. Jackson ${ }^{119}$, J.N. Jackson ${ }^{73}$, P. Jackson ${ }^{142}$, M. Jaekel ${ }^{29}$, M. Jahoda ${ }^{124}$, V. Jain ${ }^{61}$, K. Jakobs ${ }^{48}$, S. Jakobsen ${ }^{29}$, J. Jakubek ${ }^{126}$, D. Jana ${ }^{110}$, E. Jansen ${ }^{104}$, A. Jantsch ${ }^{99}$, M. Janus ${ }^{48}$, R.C. Jared ${ }^{170}$, G. Jarlskog $^{79}$, P. Jarron ${ }^{29}$, L. Jeanty ${ }^{57}$, I. Jen-La Plante ${ }^{30}$, P. Jenni ${ }^{29}$, P. Jez ${ }^{35}$, S. Jézéquel ${ }^{4}$, W. Ji ${ }^{79}$, J. Jia ${ }^{146}$, Y. Jiang ${ }^{32}$, M. Jimenez Belenguer ${ }^{29}$, G. Jin ${ }^{32}$, S. Jin ${ }^{32}$, O. Jinnouchi ${ }^{155}$, D. Joffe ${ }^{39}$, M. Johansen ${ }^{144}$, K.E. Johansson ${ }^{144}$, P. Johansson ${ }^{138}$, S. Johnert ${ }^{41}$, K.A. Johns ${ }^{6}$, K. Jon-And ${ }^{144}$, G. Jones ${ }^{82}$, R.W.L. Jones ${ }^{71}$, T.W. Jones ${ }^{77}$, T.J. Jones ${ }^{73}$, O. Jonsson ${ }^{29}$, D. Joos ${ }^{48}$, C. Joram ${ }^{29}$, P.M. Jorge ${ }^{123 b}$, V. Juranek ${ }^{124}$, P. Jussel ${ }^{62}$, V.V. Kabachenko ${ }^{127}$, S. Kabana ${ }^{16}$, M. Kaci ${ }^{165}$, A. Kaczmarska ${ }^{38}$, M. Kado ${ }^{114}$, H. Kagan ${ }^{108}$, M. Kagan ${ }^{57}$, S. Kaiser ${ }^{99}$, E. Kajomovitz ${ }^{150}$, S. Kalinin ${ }^{172}$, L.V. Kalinovskaya ${ }^{65}$, A. Kalinowski ${ }^{129}$, S. Kama ${ }^{41}$, N. Kanaya ${ }^{153}$, M. Kaneda ${ }^{153}$, V.A. Kantserov ${ }^{96}$, J. Kanzaki ${ }^{66}$, B. Kaplan ${ }^{173}$, A. Kapliy ${ }^{30}$, J. Kaplon ${ }^{29}$, M. Karagounis ${ }^{20}$, M. Karagoz Unel ${ }^{117}$, V. Kartvelishvili ${ }^{11}$, A.N. Karyukhin ${ }^{127}$, L. Kashif ${ }^{57}$, A. Kasmi ${ }^{39}$, R.D. Kass $^{108}$, A. Kastanas $^{13}$, M. Kastoryano ${ }^{173}$, M. Kataoka ${ }^{4}$, Y. Kataoka ${ }^{153}$, E. Katsoufis ${ }^{9}$, J. Katzy ${ }^{41}$, V. Kaushik ${ }^{6}$, K. Kawagoe ${ }^{67}$, T. Kawamoto ${ }^{153}$, G. Kawamura $^{81}$, M.S. Kayl ${ }^{105}$, F. Kayumov ${ }^{94}$, V.A. Kazanin ${ }^{106}$, M.Y. Kazarinov ${ }^{65}$, S.I. Kazi ${ }^{86}$, J.R. Keates ${ }^{82}$, R. Keeler ${ }^{167}$, P.T. Keener ${ }^{119}$, R. Kehoe ${ }^{39}$, M. Keil ${ }^{49}$, G.D. Kekelidze ${ }^{65}$, M. Kelly ${ }^{82}$, J. Kennedy ${ }^{98}$, M. Kenyon ${ }^{53}$,

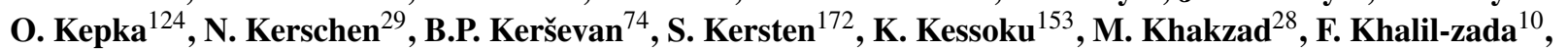
H. Khandanyan ${ }^{163}$, A. Khanov ${ }^{111}$, D. Kharchenko ${ }^{65}$, A. Khodinov ${ }^{146}$, A.G. Kholodenko ${ }^{127}$, A. Khomich ${ }^{58 a}$, G. Khoriauli $^{20}$, N. Khovanskiy ${ }^{65}$, V. Khovanskiy ${ }^{95}$, E. Khramov ${ }^{65}$, J. Khubua ${ }^{51}$, G. Kilvington ${ }^{76}$, H. Kim ${ }^{7}$, M.S. Kim ${ }^{2}$, P.C. Kim $^{142}$, S.H. Kim ${ }^{158}$, O. Kind ${ }^{15}$, P. Kind ${ }^{172}$, B.T. King ${ }^{73}$, J. Kirk ${ }^{128}$, G.P. Kirsch ${ }^{117}$, L.E. Kirsch ${ }^{22}$, A.E. Kiryunin ${ }^{99}$, D. Kisielewska ${ }^{37}$, T. Kittelmann ${ }^{122}$, H. Kiyamura ${ }^{67}$, E. Kladiva ${ }^{143}$, M. Klein ${ }^{73}$, U. Klein ${ }^{73}$, K. Kleinknecht ${ }^{81}$, M. Klemetti ${ }^{85}$, A. Klier ${ }^{169}$, A. Klimentov ${ }^{24}$, R. Klingenberg ${ }^{42}$, E.B. Klinkby ${ }^{44}$, T. Klioutchnikova ${ }^{29}$, P.F. Klok ${ }^{104}$, S. Klous ${ }^{105}$, E.-E. Kluge ${ }^{58 a}$, T. Kluge ${ }^{73}$, P. Kluit ${ }^{105}$, M. Klute ${ }^{54}$, S. Kluth ${ }^{99}$, N.S. Knecht ${ }^{156}$, E. Kneringer ${ }^{62}$, B.R. Ko ${ }^{44}$, T. Kobayashi ${ }^{153}$, M. Kobel ${ }^{43}$, B. Koblitz ${ }^{29}$, M. Kocian ${ }^{142}$, A. Kocnar ${ }^{112}$, P. Kodys ${ }^{125}$, K. Köneke ${ }^{41}$, A.C. König ${ }^{104}$, L. Köpke ${ }^{81}$, F. Koetsveld ${ }^{104}$, P. Koevesarki ${ }^{20}$, T. Koffas ${ }^{29}$, E. Koffeman ${ }^{105}$, F. Kohn ${ }^{54}$, Z. Kohout ${ }^{126}$, T. Kohriki ${ }^{66}$, T. Kokott ${ }^{20}$, H. Kolanoski ${ }^{15}$, V. Kolesnikov ${ }^{65}$, I. Koletsou ${ }^{4}$, J. Koll ${ }^{88}$, D. Kollar $^{29}$, S. Kolos ${ }^{161, q}$, S.D. Kolya ${ }^{82}$, A.A. Komar ${ }^{44}$, J.R. Komaragiri ${ }^{141}$, T. Kondo ${ }^{66}$, T. Kono ${ }^{41, p}$, A.I. Kononov $^{48}$, R. Konoplich ${ }^{107}$, S.P. Konovalov ${ }^{94}$, N. Konstantinidis ${ }^{77}$, S. Koperny ${ }^{37}$, K. Korcyl ${ }^{38}$, K. Kordas ${ }^{16}$, V. Koreshev ${ }^{127}$, A. Korn ${ }^{14}$, I. Korolkov ${ }^{11}$, E.V. Korolkova ${ }^{138}$, V.A. Korotkov ${ }^{127}$, O. Kortner ${ }^{99}$, P. Kostka ${ }^{41}$, V.V. Kostyukhin ${ }^{20}$, M.J. Kotamäki ${ }^{29}$, S. Kotov ${ }^{99}$, V.M. Kotov ${ }^{65}$, K.Y. Kotov ${ }^{106}$, Z. Koupilova ${ }^{125}$, C. Kourkoumelis ${ }^{8}$, A. Koutsman $^{105}$, R. Kowalewski ${ }^{167}$, H. Kowalski ${ }^{41}$, T.Z. Kowalski ${ }^{37}$, W. Kozanecki ${ }^{135}$, A.S. Kozhin ${ }^{127}$, V. Kral ${ }^{126}$, V.A. Kramarenko ${ }^{97}$, G. Kramberger ${ }^{74}$, M.W. Krasny ${ }^{78}$, A. Krasznahorkay ${ }^{107}$, A. Kreisel $^{151}$, F. Krejci ${ }^{126}$, A. Krepouri ${ }^{152}$, J. Kretzschmar ${ }^{73}$, P. Krieger ${ }^{156}$, G. Krobath ${ }^{98}$, K. Kroeninger ${ }^{54}$, H. Kroha ${ }^{99}$, J. Kroll ${ }^{119}$,

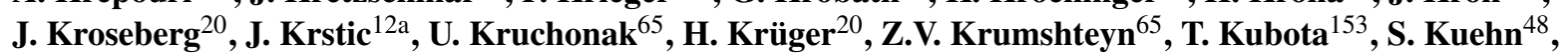

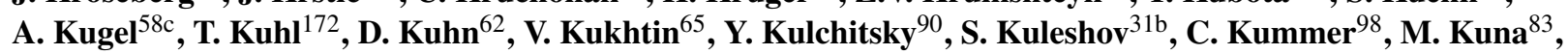
J. Kunkle $^{119}$, A. Kupco ${ }^{124}$, H. Kurashige ${ }^{67}$, M. Kurata ${ }^{158}$, L.L. Kurchaninov ${ }^{157 a}$, Y.A. Kurochkin ${ }^{90}$, V. Kus ${ }^{124}$, E. Kuznetsovaa ${ }^{131 a, 131 b}$, O. Kvasnicka ${ }^{124}$, R. Kwee ${ }^{15}$, L. La Rotonda ${ }^{36 a, 36 b}$, L. Labarga ${ }^{80}$, J. Labbe ${ }^{4}$, C. Lacasta ${ }^{165}$,

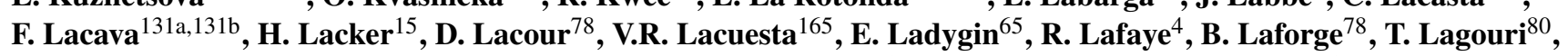

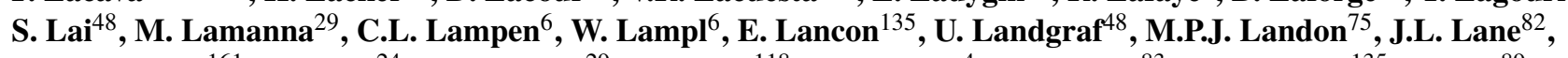

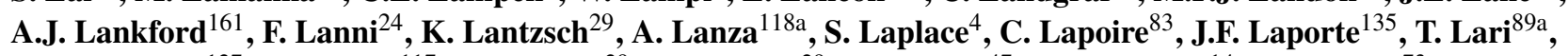

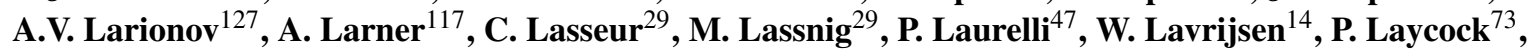
A.B. Lazarev ${ }^{65}$, A. Lazzaro ${ }^{89 a, 89 b}$, O. Le Dortz ${ }^{78}$, E. Le Guirriec ${ }^{83}$, C. Le Maner ${ }^{156}$, E. Le Menedeu ${ }^{135}$, M. Le Vine ${ }^{24}$,

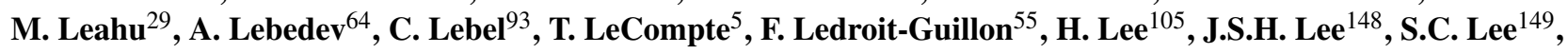




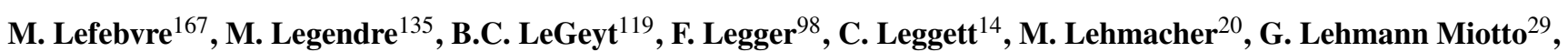
X. Lei ${ }^{6}$, R. Leitner ${ }^{125}$, D. Lelas ${ }^{167}$, D. Lellouch ${ }^{169}$, J. Lellouch ${ }^{78}$, M. Leltchouk ${ }^{34}$, V. Lendermann ${ }^{58 a}$, K.J.C. Leney ${ }^{73}$, T. Lenz ${ }^{172}$, G. Lenzen ${ }^{172}$, B. Lenzi ${ }^{135}$, K. Leonhardt ${ }^{43}$, C. Leroy ${ }^{93}$, J.-R. Lessard ${ }^{167}$, C.G. Lester ${ }^{27}$, A. Leung Fook Cheong $^{170}$, J. Levêque ${ }^{83}$, D. Levin ${ }^{87}$, L.J. Levinson ${ }^{169}$, M.S. Levitski ${ }^{127}$, S. Levonian ${ }^{41}$, M. Lewandowska ${ }^{21}$,

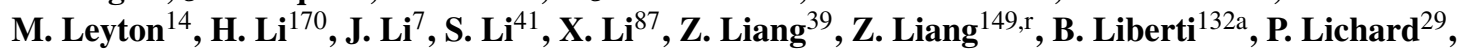
M. Lichtnecker ${ }^{98}$, K. Lie ${ }^{163}$, W. Liebig ${ }^{105}$, D. Liko ${ }^{29}$, J.N. Lilley ${ }^{17}$, H. Lim ${ }^{5}$, A. Limosani ${ }^{86}$, M. Limper ${ }^{63}$, S.C. Lin $^{149}$, S.W. Lindsay ${ }^{73}$, V. Linhart ${ }^{126}$, J.T. Linnemann ${ }^{88}$, A. Liolios ${ }^{152}$, E. Lipeles ${ }^{119}$, L. Lipinsky ${ }^{124}$,

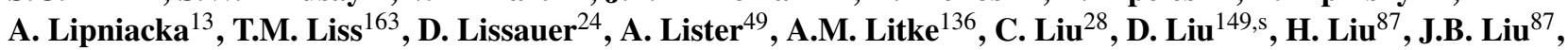

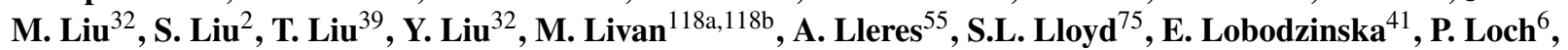
W.S. Lockman ${ }^{136}$, S. Lockwitz ${ }^{173}$, T. Loddenkoetter ${ }^{20}$, F.K. Loebinger ${ }^{82}$, A. Loginov ${ }^{173}$, C.W. Loh ${ }^{166}$, T. Lohse ${ }^{15}$,

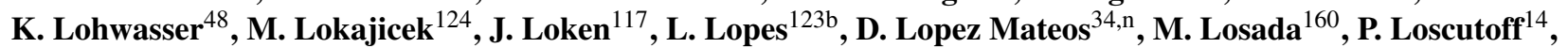

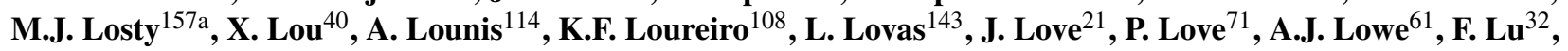

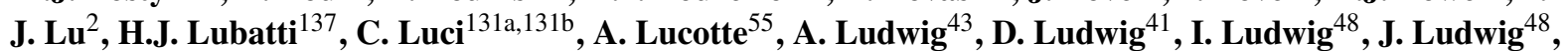
F. Luehring ${ }^{61}$, L. Luisa ${ }^{162 a, 162 c}$, D. Lumb ${ }^{48}$, L. Luminari ${ }^{131 a}$, E. Lund ${ }^{116}$, B. Lund-Jensen ${ }^{145}$, B. Lundberg $^{79}$, J. Lundberg ${ }^{29}$, J. Lundquist ${ }^{35}$, G. Lutz ${ }^{99}$, D. Lynn ${ }^{24}$, J. Lys ${ }^{14}$, E. Lytken ${ }^{79}$, H. Ma ${ }^{24}$, L.L. Ma ${ }^{170}$, J.A. Macana Goia $^{93}$, G. Maccarrone ${ }^{47}$, A. Macchiolo ${ }^{99}$, B. Maček ${ }^{74}$, J. Machado Miguens ${ }^{123 b}$, R. Mackeprang ${ }^{29}$, R.J. Madaras $^{14}$, W.F. Mader $^{43}$, R. Maenner ${ }^{58 c}$, T. Maeno ${ }^{24}$, P. Mättig ${ }^{172}$, S. Mättig ${ }^{41}$, P.J. Magalhaes Martins ${ }^{123 b}$, E. Magradze ${ }^{51}$, C.A. Magrath ${ }^{104}$, Y. Mahalalel ${ }^{151}$, K. Mahboubi ${ }^{48}$, A. Mahmood $^{1}$, G. Mahout $^{17}$, C. Maiani $^{131 \mathrm{a}, 131 \mathrm{~b}}$, C. Maidantchik ${ }^{23 a}$, A. Maio ${ }^{123 b}$, S. Majewski ${ }^{24}$, Y. Makida ${ }^{66}$, M. Makouski ${ }^{127}$, N. Makovec $^{114}$, Pa. Malecki ${ }^{38}$, P. Malecki ${ }^{38}$, V.P. Maleev $^{120}$, F. Malek ${ }^{55}$, U. Mallik ${ }^{63}$, D. Malon ${ }^{5}$, S. Maltezos ${ }^{9}$, V. Malyshev ${ }^{106}$, S. Malyukov ${ }^{65}$,

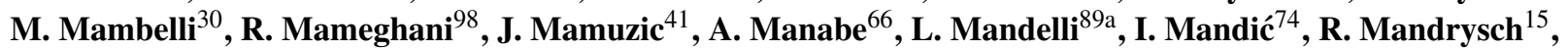
J. Maneira ${ }^{123 b}$, P.S. Mangeard ${ }^{88}$, I.D. Manjavidze ${ }^{65}$, P.M. Manning ${ }^{136}$, A. Manousakis-Katsikakis ${ }^{8}$, B. Mansoulie $^{135}$,

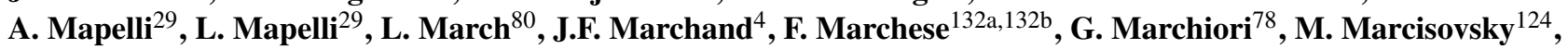
C.P. Marino $^{61}$, C.N. Marques ${ }^{123 b}$, F. Marroquim ${ }^{23 a}$, R. Marshall ${ }^{82}$, Z. Marshall $^{\text {34,n }}$, F.K. Martens ${ }^{156}$, S. Marti i

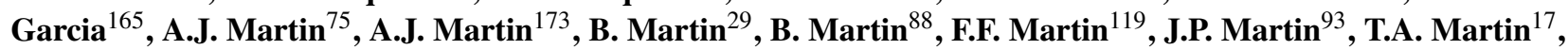
B. Martin dit Latour ${ }^{49}$, M. Martinez ${ }^{11}$, V. Martinez Outschoorn ${ }^{57}$, A. Martini ${ }^{47}$, A.C. Martyniuk ${ }^{82}$,

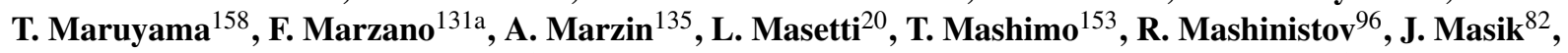
A.L. Maslennikov ${ }^{106}$, G. Massaro ${ }^{105}$, N. Massol $^{4}$, A. Mastroberardino ${ }^{36 a, 36 b}$, T. Masubuchi ${ }^{153}$, M. Mathes $^{20}$, P. Matricon ${ }^{114}$, H. Matsunaga ${ }^{153}$, T. Matsushita ${ }^{67}$, C. Mattravers $^{117, t}$, S.J. Maxfield ${ }^{73}$, E.N. May ${ }^{5}$, A. Mayne ${ }^{138}$, R. Mazini ${ }^{149}$, M. Mazur ${ }^{48}$, M. Mazzanti ${ }^{89 a}$, P. Mazzanti ${ }^{19 a}$, J. Mc Donald ${ }^{85}$, S.P. Mc Kee ${ }^{87}$, A. McCarn ${ }^{163}$, R.L. McCarthy ${ }^{146}$, N.A. McCubbin ${ }^{128}$, K.W. McFarlane ${ }^{56}$, H. McGlone ${ }^{53}$, G. Mchedlidze $^{51}$, R.A. McLaren ${ }^{29}$, S.J. McMahon ${ }^{128}$, T.R. McMahon ${ }^{76}$, R.A. McPherson ${ }^{167, h}$, A. Meade ${ }^{84}$, J. Mechnich ${ }^{105}$, M. Mechtel ${ }^{172}$, M. Medinnis ${ }^{41}$, R. Meera-Lebbai ${ }^{110}$, T.M. Meguro ${ }^{115}$, R. Mehdiyev ${ }^{93}$, S. Mehlhase ${ }^{41}$, A. Mehta ${ }^{73}$, K. Meier ${ }^{58 a}$, B. Meirose $^{48}$, C. Melachrinos ${ }^{30}$, A. Melamed-Katz ${ }^{169}$, B.R. Mellado Garcia ${ }^{170}$, Z. Meng ${ }^{149, \text { u }}$, S. Menke ${ }^{99}$, E. Meoni ${ }^{11}$, D. Merki $^{98}$, P. Mermod ${ }^{117}$, L. Merola ${ }^{102 a, 102 b}$, C. Meroni ${ }^{89 a}$, F.S. Merritt ${ }^{30}$, A.M. Messina ${ }^{29}$, I. Messmer ${ }^{48}$, J. Metcalfe ${ }^{103}$, A.S. Mete ${ }^{64}$, J.-P. Meyer ${ }^{135}$, J. Meyer ${ }^{171}$, J. Meyer ${ }^{54}$, T.C. Meyer ${ }^{29}$, W.T. Meyer ${ }^{64}$,

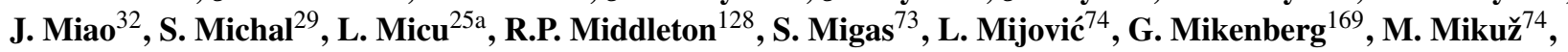
D.W. Miller ${ }^{142}$, W.J. Mills ${ }^{166}$, C.M. Mills ${ }^{57}$, A. Milov ${ }^{169}$, D.A. Milstead ${ }^{144}$, A.A. Minaenko ${ }^{127}$, M. Miñano ${ }^{165}$, I.A. Minashvili ${ }^{65}$, A.I. Mincer ${ }^{107}$, B. Mindur ${ }^{37}$, M. Mineev ${ }^{65}$, Y. Ming ${ }^{129}$, L.M. Mir ${ }^{11}$, G. Mirabelli ${ }^{131 a}$, S. Misawa ${ }^{24}$, S. Miscetti ${ }^{47}$, A. Misiejuk $^{76}$, J. Mitrevski ${ }^{136}$, V.A. Mitsou ${ }^{165}$, P.S. Miyagawa ${ }^{82}$, J.U. Mjörnmark ${ }^{79}$, D. Mladenov ${ }^{22}$, T. Moa $^{144}$, S. Moed $^{57}$, V. Moeller ${ }^{27}$, K. Mönig ${ }^{41}$, N. Möser ${ }^{20}$, B. Mohn ${ }^{13}$, W. Mohr ${ }^{48}$, S. Mohrdieck-Möck ${ }^{99}$, R. Moles-Valls ${ }^{165}$, J. Molina-Perez ${ }^{29}$, G. Moloney ${ }^{86}$, J. Monk ${ }^{77}$, E. Monnier ${ }^{83}$, S. Montesano $^{89 a, 89 b}$, F. Monticelli $^{70}$, R.W. Moore ${ }^{2}$, C. Mora Herrera ${ }^{49}$, A. Moraes ${ }^{53}$, A. Morais ${ }^{123 b}$, J. Morel ${ }^{4}$, G. Morello ${ }^{36,36 b}$, D. Moreno ${ }^{160}$, M. Moreno Llácer ${ }^{165}$, P. Morettini ${ }^{50 a}$, M. Morii ${ }^{57}$, A.K. Morley ${ }^{86}$, G. Mornacchi $^{29}$, S.V. Morozov ${ }^{96}$, J.D. Morris $^{75}$, H.G. Moser ${ }^{99}$, M. Mosidze $^{51}$, J. Moss ${ }^{108}$, R. Mount ${ }^{142}$, E. Mountricha ${ }^{9}$, S.V. Mouraviev ${ }^{94}$, E.J.W. Moyse ${ }^{84}$, M. Mudrinic ${ }^{12 b}$, F. Mueller ${ }^{58 a}$, J. Mueller ${ }^{122}$, K. Mueller ${ }^{20}$, T.A. Müller ${ }^{8}$, D. Muenstermann ${ }^{42}$, A. Muir M $^{166}$, Y. Munwes $^{151}$, R. Murillo Garcia ${ }^{161}$, W.J. Murray ${ }^{128}$, I. Mussche ${ }^{105}$, E. Musto ${ }^{102 a, 102 b}$, A.G. Myagkov ${ }^{127}$,

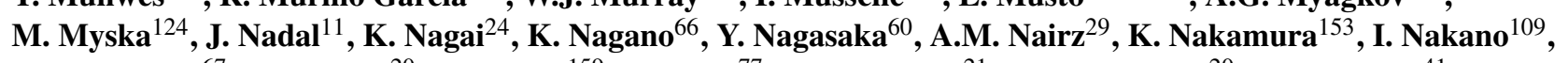
H. Nakatsuka ${ }^{67}$, G. Nanava ${ }^{20}$, A. Napier ${ }^{159}$, M. Nash ${ }^{77, v}$, N.R. Nation ${ }^{21}$, T. Nattermann ${ }^{20}$, T. Naumann ${ }^{41}$,

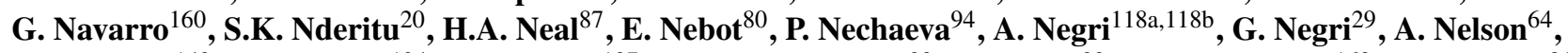
T.K. Nelson ${ }^{142}$, S. Nemecek ${ }^{124}$, P. Nemethy ${ }^{107}$, A.A. Nepomuceno ${ }^{23 a}$, M. Nessi ${ }^{29}$, M.S. Neubauer $^{163}$, A. Neusiedl ${ }^{81}$, R.N. Neves ${ }^{123 b}$, P. Nevski ${ }^{24}$, F.M. Newcomer ${ }^{119}$, R.B. Nickerson ${ }^{117}$, R. Nicolaidou ${ }^{135}$, L. Nicolas ${ }^{138}$, G. Nicoletti ${ }^{47}$, 
F. Niedercorn ${ }^{114}$, J. Nielsen ${ }^{136}$, A. Nikiforov ${ }^{15}$, K. Nikolaev ${ }^{65}$, I. Nikolic-Audit $^{78}$, K. Nikolopoulos $^{8}$, H. Nilsen ${ }^{48}$, P. Nilsson ${ }^{7}$, A. Nisati ${ }^{131}{ }^{\text {, T. Nishiyama }}{ }^{67}$, R. Nisius ${ }^{99}$, L. Nodulman ${ }^{5}$, M. Nomachi ${ }^{115}$ I. Nomidis $^{152}$, M. Nordberg ${ }^{29}$, B. Nordkvist ${ }^{144}$, D. Notz ${ }^{41}$, J. Novakova ${ }^{125}$, M. Nozaki ${ }^{66}$, M. Nožička ${ }^{41}$, I.M. Nugent ${ }^{157 a}$, A.-E. Nuncio-Quiroz ${ }^{20}$, G. Nunes Hanninger $^{20}$, T. Nunnemann ${ }^{98}$, E. Nurse $^{77}$, D.C. O'Neil ${ }^{141}$, V. O'Shea ${ }^{53}$, F.G. Oakham ${ }^{28, b}$, H. Oberlack ${ }^{99}$, A. Ochi ${ }^{67}$, S. Oda ${ }^{153}$, S. Odaka ${ }^{66}$, J. Odier ${ }^{83}$, G.A. Odino ${ }^{50 a, 50 b}$, H. Ogren ${ }^{61}$, A. Oh ${ }^{82}$, S.H. Oh ${ }^{44}$, C.C. Ohm ${ }^{144}$, T. Ohshima ${ }^{101}$, H. Ohshita ${ }^{139}$, T. Ohsugi ${ }^{59}$, S. Okada ${ }^{67}$, H. Okawa ${ }^{153}$, Y. Okumura ${ }^{101}$, M. Olcese ${ }^{50 a}$,

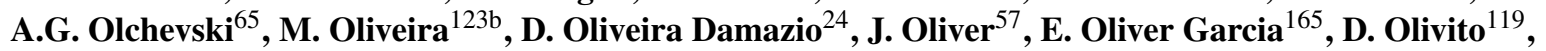
A. Olszewski ${ }^{38}$, J. Olszowska ${ }^{38}$, C. Omachi ${ }^{67}$, A. Onofre ${ }^{123 b}$, P.U.E. Onyisi ${ }^{30}$, C.J. Oram ${ }^{157 a}$, G. Ordonez ${ }^{104}$, M.J. Oreglia ${ }^{30}$, Y. Oren ${ }^{151}$, D. Orestano ${ }^{133 a}$,133b , I. Orlov ${ }^{106}$, C. Oropeza Barrera ${ }^{53}$, R.S. Orr ${ }^{156}$, E.O. Ortega ${ }^{129}$,

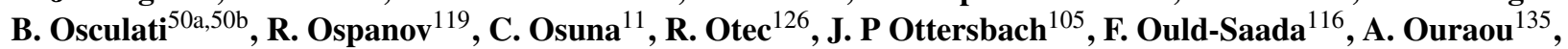
Q. Ouyang ${ }^{32}$, M. Owen ${ }^{82}$, S. Owen ${ }^{138}$, A. Oyarzun ${ }^{31 b}$, V.E. Ozcan ${ }^{77}$, K. Ozone ${ }^{66}$, N. Ozturk ${ }^{7}$, A. Pacheco Pages ${ }^{11}$, S. Padhi ${ }^{170}$, C. Padilla Aranda ${ }^{11}$, E. Paganis ${ }^{138}$, C. Pahl ${ }^{63}$, F. Paige ${ }^{24}$, K. Pajchel ${ }^{116}$, S. Palestini ${ }^{29}$, D. Pallin ${ }^{33}$, A. Palma ${ }^{123 b}$, J.D. Palmer ${ }^{17}$, Y.B. Pan ${ }^{170}$, E. Panagiotopoulou ${ }^{9}$, B. Panes ${ }^{31 a}$, N. Panikashvili ${ }^{87}$, S. Panitkin ${ }^{24}$, D. Pantea ${ }^{25 a}$, M. Panuskova ${ }^{124}$, V. Paolone ${ }^{122}$, Th.D. Papadopoulou' ${ }^{9}$ S.J. Park ${ }^{54}$, W. Park ${ }^{24, w}$, M.A. Parker ${ }^{27}$, S.I. Parker ${ }^{14}$, F. Parodi ${ }^{50 a, 50 b}$, J.A. Parsons ${ }^{34}$, U. Parzefall ${ }^{48}$, E. Pasqualucci ${ }^{131 a}{ }^{\text {, G. } \text { Passardi }^{29} \text {, A. Passeri }}{ }^{133 a}$, F. Pastore ${ }^{133 a, 133 b}$, Fr. Pastore ${ }^{29}$, G. Pásztor ${ }^{49, x}$, S. Pataraia ${ }^{99}$, J.R. Pater ${ }^{82}$, S. Patricelli ${ }^{102 a, 102 b}$, A. Patwa $^{24}$, T. Pauly ${ }^{29}$, L.S. Peak ${ }^{148}$, M. Pecsy ${ }^{143}$, M.I. Pedraza Morales ${ }^{170}$, S.V. Peleganchuk ${ }^{106}$, H. Peng ${ }^{170}$, A. Penson ${ }^{34}$, J. Penwell ${ }^{61}$, M. Perantoni ${ }^{23 a}$, K. Perez ${ }^{34, n}$, E. Perez Codina ${ }^{11}$, M.T. Pérez García-Estañ ${ }^{165}$, V. Perez Reale ${ }^{34}$, L. Perini ${ }^{89 a, 89 b}$, H. Pernegger ${ }^{29}$, R. Perrino ${ }^{72 a}$, P. Perrodo ${ }^{4}$, S. Persembe ${ }^{3}$, P. Perus ${ }^{114}$, V.D. Peshekhonov ${ }^{65}$, B.A. Petersen ${ }^{29}$, J. Petersen ${ }^{29}$, T.C. Petersen ${ }^{35}$, E. Petit ${ }^{83}$, C. Petridou ${ }^{152}$, E. Petrolo ${ }^{131 a}$, F. Petrucci ${ }^{133 a, 133 b}$, D. Petschull ${ }^{41}$, M. Petteni ${ }^{141}$, R. Pezoa ${ }^{31 b}$, B. Pfeifer $^{48}$, A. Phan ${ }^{86}$, A.W. Phillips ${ }^{27}$, G. Piacquadio ${ }^{48}$, M. Piccinini ${ }^{19 a, 19 b}$, R. Piegaia ${ }^{26}$, J.E. Pilcher ${ }^{30}$, A.D. Pilkington ${ }^{82}$, J. Pina ${ }^{123 b}$, M. Pinamonti ${ }^{162 a, 162 c}$, J.L. Pinfold ${ }^{2}$, J. Ping ${ }^{32}$, B. Pinto ${ }^{123 b}$, C. Pizio ${ }^{89}$, 89 b , R. Placakyte ${ }^{41}$, M. Plamondon ${ }^{167}$, W.G. Plano ${ }^{82}$, M.-A. Pleier ${ }^{24}$,

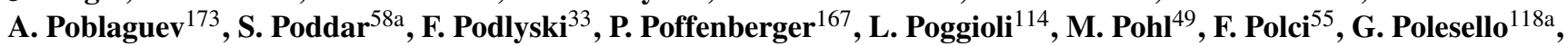
A. Policicchio ${ }^{137}$, A. Polini ${ }^{19 a}$, J. Poll ${ }^{75}$, V. Polychronakos ${ }^{24}$, D.M. Pomarede ${ }^{135}$, D. Pomeroy ${ }^{22}$, K. Pommès ${ }^{29}$, L. Pontecorvo ${ }^{131 a}$, B.G. Pope ${ }^{88}$, D.S. Popovic ${ }^{12 a}$, A. Poppleton ${ }^{29}$, J. Popule ${ }^{124}$, X. Portell Bueso ${ }^{48}$, R. Porter ${ }^{161}$, G.E. Pospelov ${ }^{99}$, P. Pospichal ${ }^{29}$, S. Pospisil ${ }^{126}$, M. Potekhin ${ }^{24}$, I.N. Potrap ${ }^{99}$, C.J. Potter ${ }^{147}$, C.T. Potter ${ }^{85}$, K.P. Potter ${ }^{82}$, G. Poulard ${ }^{29}$, J. Poveda ${ }^{170}$, R. Prabhu ${ }^{20}$, P. Pralavorio ${ }^{83}$, S. Prasad ${ }^{57}$, R. Pravahan ${ }^{7}$, T. Preda ${ }^{25 a}$, K. Pretzl $^{16}$, L. Pribyl ${ }^{29}$, D. Price ${ }^{61}$, L.E. Price ${ }^{5}$, P.M. Prichard ${ }^{73}$, D. Prieur ${ }^{122}$, M. Primavera ${ }^{72 a}$, K. Prokofiev ${ }^{29}$,

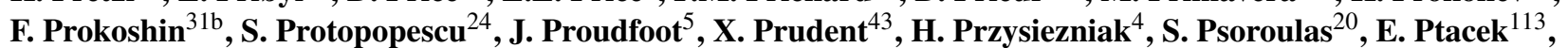
C. Puigdengoles ${ }^{11}$, J. Purdham ${ }^{87}$, M. Purohit ${ }^{24, w}$, P. Puzo ${ }^{114}$, Y. Pylypchenko ${ }^{116}$, M. Qi ${ }^{32}$, J. Qian ${ }^{87}$, W. Qian ${ }^{128}$, Z. Qian ${ }^{83}$, Z. Qin ${ }^{41}$, D. Qing ${ }^{157}$, A. Quadt ${ }^{54}$, D.R. Quarrie ${ }^{14}$, W.B. Quayle ${ }^{170}$, F. Quinonez ${ }^{31 a}$, M. Raas ${ }^{104}$,

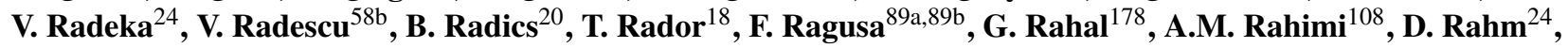
S. Rajagopalan $^{24}$, M. Rammes ${ }^{140}$, P.N. Ratoff ${ }^{71}$, F. Rauscher $^{98}$, E. Rauter $^{99}$, M. Raymond ${ }^{29}$, A.L. Read ${ }^{116}$, D.M. Rebuzzi ${ }^{18 a, 118 b}$, A. Redelbach ${ }^{171}$, G. Redlinger $^{24}$, R. Reece $^{119}$, K. Reeves ${ }^{40}$, E. Reinherz-Aronis ${ }^{151}$, A. Reinsch $^{113}$, I. Reisinger ${ }^{42}$, D. Reljic ${ }^{12 a}$, C. $\operatorname{Rembser}^{29}$, Z.L. Ren ${ }^{149}$, P. $_{\text {Renkel }}{ }^{39}$, S. $_{\text {Rescia }}{ }^{24}$, M. Rescigno $^{131 a}$, S. Resconi $^{89}$, B. Resende ${ }^{105}$, P. Reznicek ${ }^{125}$, R. Rezvani ${ }^{156}$, A. Richards ${ }^{77}$, R.A. Richards ${ }^{88}$, R. Richter ${ }^{99}$, E. Richter-Was ${ }^{38, y}$, M. Ridel $^{78}$, S. Rieke ${ }^{81}$, M. Rijpstra ${ }^{105}$, M. Rijssenbeek ${ }^{146}$, A. Rimoldi ${ }^{118 a, 118 b}$, L. Rinaldi ${ }^{19 a}$, R.R. Rios $^{39}$, I. Riu ${ }^{11}$, G. Rivoltella ${ }^{89 a, 89 b}$, F. Rizatdinova ${ }^{111}$, E.R. Rizvi ${ }^{75}$, D.A. Roa Romero ${ }^{160}$, S.H. Robertson ${ }^{85, h}$, A. Robichaud-Veronneau ${ }^{49}$, D. Robinson ${ }^{27}$, J. Robinson ${ }^{77}$, M. Robinson ${ }^{113}$, A. Robson ${ }^{53}$, J.G. Rocha de Lima ${ }^{5}$, C. Roda ${ }^{121 a, 121 b}$, D. Roda Dos Santos ${ }^{29}$, D. Rodriguez ${ }^{160}$, Y. Rodriguez Garcia ${ }^{15}$, S. Roe ${ }^{29}$, O. Røhne ${ }^{116}$, V. Rojo ${ }^{1}$, S. Rolli ${ }^{159}$, A. Romaniouk ${ }^{96}$, V.M. Romanov ${ }^{65}$, G. Romeo ${ }^{26}$, D. Romero Maltrana ${ }^{31 a}$, L. $\operatorname{Roos}^{78}$, E. Ros ${ }^{165}$,

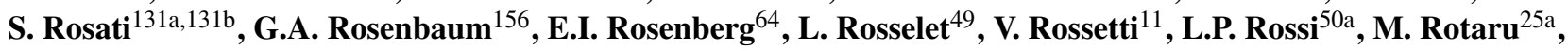
J. Rothberg ${ }^{137}$, I. Rottländer ${ }^{20}$, D. Rousseau ${ }^{114}$, C.R. $\operatorname{Royon}^{135},{\text { A. } \text { Rozanov }^{83}, \text { Y. }_{\text {Rozen }}{ }^{150}, \text { X. Ruan }}^{114}$, B. Ruckert $^{98}$, N. Ruckstuhl ${ }^{105}$, V.I. Rud ${ }^{97}$, G. Rudolph $^{62}$, F. Rühr ${ }^{58 a}$, F. Ruggieri ${ }^{133 a}$, A. Ruiz-Martinez ${ }^{64}$, L. Rumyantsev $^{65}$, N.A. Rusakovich ${ }^{65}$, J.P. Rutherfoord ${ }^{6}$, C. Ruwiedel $^{20}$, P. Ruzicka ${ }^{124}$, Y.F. Ryabov ${ }^{120}$, V. Ryadovikov ${ }^{127}$, P. Ryan $^{88}$, G. Rybkin ${ }^{114}$, S. Rzaeva ${ }^{10}$, A.F. Saavedra ${ }^{148}$, H.F.-W. Sadrozinski ${ }^{136}$, R. Sadykov ${ }^{65}$, H. Sakamoto ${ }^{153}$, G. Salamanna ${ }^{105}$, A. Salamon ${ }^{132 a}$, M. Saleem ${ }^{110}$, D. Salihagic ${ }^{99}$, A. Salnikov ${ }^{142}$, J. Salt ${ }^{165}$, B.M. Salvachua Ferrando ${ }^{5}$, D. Salvatore ${ }^{36 a, 36 b}$, F. Salvatore ${ }^{147}$, A. Salvucci ${ }^{47}$, A. Salzburger $^{29}$, D. Sampsonidis ${ }^{152}$, B.H. Samset ${ }^{116}$, M.A. Sanchis Lozano ${ }^{165}$, H. Sandaker ${ }^{13}$, H.G. Sander ${ }^{81}$, M.P. Sanders ${ }^{98}$, M. Sandhoff ${ }^{172}$, R. Sandstroem ${ }^{105}$, S. Sandvoss ${ }^{172}$, D.P.C. Sankey ${ }^{128}$, B. Sanny ${ }^{172}$, A. Sansoni ${ }^{47}$, C. Santamarina Rios ${ }^{85}$, L. Santi ${ }^{162 a, 162 c}$, C. Santoni ${ }^{33}$, R. Santonico ${ }^{132 a, 132 b}$, J. Santos ${ }^{123 b}$, J.G. Saraiva ${ }^{123 b}$, T. Sarangi ${ }^{170}$, 
E. Sarkisyan-Grinbaum ${ }^{7}$, F. Sarri ${ }^{121 a, 121 b}$, O. Sasaki ${ }^{66}$, T. Sasaki ${ }^{66}$, N. Sasao ${ }^{68}$, I. Satsounkevitch ${ }^{90}$, G. Sauvage ${ }^{4}$, P. Savard ${ }^{156, b}$, A.Y. Savine ${ }^{6}$, V. Savinov ${ }^{122}$, L. Sawyer ${ }^{24, i}$, D.H. Saxon ${ }^{53}$, L.P. Says ${ }^{33}$, C. Sbarra ${ }^{19 a, 19 b}$, A. Sbrizzi ${ }^{19 a, 19 b}$, D.A. Scannicchio ${ }^{29}$, J. Schaarschmidt ${ }^{43}$, P. Schacht ${ }^{99}$, U. Schäfer ${ }^{81}$, S. Schaetzel ${ }^{58 b}$, A.C. Schaffer ${ }^{114}$, D. Schaile ${ }^{98}$, R.D. Schamberger ${ }^{146}$, A.G. Schamov ${ }^{106}$, V.A. Schegelsky ${ }^{120}$, D. Scheirich ${ }^{87}$, M. Schernau ${ }^{161}$, M.I. Scherzer ${ }^{14}$, C. Schiavi ${ }^{50 a, 50 b}$, J. Schieck ${ }^{99}$, M. Schioppa ${ }^{36,36 b}$, S. Schlenker ${ }^{29}$, J.L. Schlereth ${ }^{5}$, P. Schmid ${ }^{62}$, K. Schmieden ${ }^{20}$, C. Schmitt ${ }^{81}$, M. Schmitz ${ }^{20}$, M. Schott ${ }^{29}$, D. Schouten ${ }^{141}$, J. Schovancova ${ }^{124}$, M. Schram ${ }^{85}$, A. Schreiner ${ }^{63}$, C. Schroeder ${ }^{81}$, N. Schroer ${ }^{58 c}$, M. Schroers ${ }^{172}$, G. Schuler ${ }^{29}$, J. Schultes ${ }^{172}$, H.-C. Schultz-Coulon ${ }^{58 a}$, J.W. Schumacher ${ }^{43}$, M. Schumacher ${ }^{48}$, B.A. Schumm ${ }^{136}$, Ph. Schune ${ }^{135}$, C. Schwanenberger ${ }^{82}$, A. Schwartzman ${ }^{142}$, Ph. Schwemling ${ }^{78}$, R. Schwienhorst ${ }^{88}$, R. Schwierz ${ }^{43}$, J. Schwindling ${ }^{135}$, W.G. Scott $^{128}$, J. Searcy ${ }^{113}$, E. Sedykh ${ }^{120}$, E. Segura ${ }^{11}$, S.C. Seidel ${ }^{103}$, A. Seiden ${ }^{136}$, F. Seifert ${ }^{43}$, J.M. Seixas ${ }^{23 a}$, G. Sekhniaidze ${ }^{102 a}$, D.M. Seliverstov ${ }^{120}$, B. Sellden ${ }^{144}$, M. Seman ${ }^{143}$, N. Semprini-Cesari ${ }^{19 a}, 19 b$, C. Serfon ${ }^{98}$, L. Serin ${ }^{114}$, R. Seuster ${ }^{99}$, H. Severini ${ }^{110}$, M.E. Sevior ${ }^{86}$, A. Sfyrla ${ }^{163}$, E. Shabalina ${ }^{54}$, M. Shamim ${ }^{113}$, L.Y. Shan ${ }^{32}$, J.T. Shank ${ }^{21}$, Q.T. Shao ${ }^{86}$, M. Shapiro ${ }^{14}$, P.B. Shatalov ${ }^{95}$, L. Shaver ${ }^{6}$, K. Shaw ${ }^{138}$, D. Sherman ${ }^{29}$, P. Sherwood ${ }^{77}$, A. Shibata ${ }^{107}$, M. Shimojima ${ }^{100}$, T. Shin ${ }^{56}$, A. Shmeleva ${ }^{94}$, M.J. Shochet ${ }^{30}$, M.A. Shupe ${ }^{6}$, P. Sicho ${ }^{124}$, A. Sidoti ${ }^{15}$, A. Siebel $^{172}$, F. Siegert $^{77}$, J. Siegrist $^{14}$, Dj. Sijacki ${ }^{12 a}$, O. Silbert ${ }^{169}$, J. Silva ${ }^{123 b}$, Y. Silver ${ }^{151}$, D. Silverstein ${ }^{142}$, S.B. Silverstein ${ }^{144}$, V. Simak ${ }^{126}$, Lj. Simic $^{12 a}$, S. Simion ${ }^{114}$, B. Simmons ${ }^{77}$, M. Simonyan ${ }^{4}$, P. Sinervo ${ }^{156}$, N.B. Sinev ${ }^{113}$, V. Sipica ${ }^{140}$, G. Siragusa ${ }^{81}$, A.N. Sisakyan ${ }^{65}$, S.Yu. Sivoklokov ${ }^{97}$, J. Sjoelin ${ }^{144}$, T.B. Sjursen ${ }^{13}$, P. Skubic ${ }^{110}$, N. Skvorodnev ${ }^{22}$, M. Slater ${ }^{17}$, T. Slavicek ${ }^{126}$, K. Sliwa ${ }^{159}$, J. Sloper ${ }^{29}$, T. Sluka ${ }^{124}$, V. Smakhtin ${ }^{169}$, S.Yu. Smirnov ${ }^{96}$, Y. Smirnov ${ }^{24}$, L.N. Smirnova ${ }^{97}$, O. Smirnova ${ }^{79}$, B.C. Smith ${ }^{57}$, D. Smith ${ }^{142}$, K.M. Smith ${ }^{53}$, M. Smizanska ${ }^{71}$, K. Smolek ${ }^{126}$, A.A. Snesarev ${ }^{94}$, S.W. Snow ${ }^{82}$, J. Snow ${ }^{110}$, J. Snuverink ${ }^{105}$, S. Snyder ${ }^{24}$, M. Soares ${ }^{123 b}$, R. Sobie ${ }^{167, h}$, J. Sodomka ${ }^{126}$, A. Soffer ${ }^{151}$, C.A. Solans ${ }^{165}$, M. Solar ${ }^{126}$, J. Solc ${ }^{126}$, E. Solfaroli Camillocci ${ }^{131 a, 131 b}$, A.A. Solodkov ${ }^{127}$, O.V. Solovyanov ${ }^{127}$, R. Soluk ${ }^{2}$, J. Sondericker ${ }^{24}$, V. Sopko ${ }^{126}$, B. Sopko ${ }^{126}$, M. Sosebee ${ }^{7}$, V.V. Sosnovtsev ${ }^{96}$, L. Sospedra Suay ${ }^{165}$, A. Soukharev ${ }^{106}$, S. Spagnolo ${ }^{72 a, 72 b}$, F. Spanò ${ }^{34}$, P. Speckmayer ${ }^{29}$, E. Spencer ${ }^{136}$, R. Spighi ${ }^{19 a}$, G. Spigo $^{29}$, F. Spila ${ }^{131 a, 131 b}$, R. Spiwoks ${ }^{29}$, M. Spousta ${ }^{125}$, T. Spreitzer ${ }^{141}$, B. Spurlock ${ }^{7}$, R.D.St. Denis ${ }^{53}$, T. Stahl ${ }^{140}$, J. Stahlman ${ }^{119}$, R. Stamen ${ }^{58 a}$, S.N. Stancu ${ }^{161}$, E. Stanecka ${ }^{29}$, R.W. Stanek ${ }^{5}$, C. Stanescu ${ }^{133 a}$, S. Stapnes ${ }^{116}$, E.A. Starchenko ${ }^{127}$, J. Stark ${ }^{55}$, P. Staroba ${ }^{124}$, P. Starovoitov ${ }^{91}$, J. Stastny ${ }^{124}$, A. Staude ${ }^{98}$, P. Stavina ${ }^{143}$, G. Stavropoulos ${ }^{14}$, G. Steele ${ }^{53}$, P. Steinbach ${ }^{43}$, P. Steinberg ${ }^{24}$, I. Stekl ${ }^{126}$, B. Stelzer ${ }^{141}$, H.J. Stelzer ${ }^{41}$, O. Stelzer-Chilton ${ }^{157 a}$, H. Stenzel ${ }^{52}$, K. Stevenson ${ }^{75}$, G. Stewart ${ }^{53}$, M.C. Stockton ${ }^{29}$, K. Stoerig ${ }^{48}$, G. Stoicea ${ }^{25 a}$, S. Stonjek ${ }^{99}$, P. Strachota ${ }^{125}$, A. Stradling ${ }^{7}$, A. Straessner ${ }^{43}$, J. Strandberg ${ }^{87}$, S. Strandberg ${ }^{14}$, A. Strandlie ${ }^{116}$, M. Strauss ${ }^{110}$, P. Strizenec ${ }^{143}$, R. Ströhmer ${ }^{98}$, D.M. Strom ${ }^{113}$, J.A. Strong ${ }^{76, *}$, R. Stroynowski ${ }^{39}$, J. Strube ${ }^{128}$, B. Stugu ${ }^{13}$, I. Stumer ${ }^{24, *}$, D.A. Soh $^{149, \mathrm{r}}$, D. Su ${ }^{142}$, S.I. Suchkov ${ }^{96}$, Y. Sugaya ${ }^{115}$, T. Sugimoto ${ }^{101}$, C. Suhr ${ }^{5}$, M. Suk ${ }^{125}$, V.V. Sulin ${ }^{44}$, S. Sultansoy ${ }^{3, z}$, T. Sumida ${ }^{29}$, X. Sun ${ }^{32}$, J.E. Sundermann ${ }^{48}$, K. Suruliz ${ }^{162 a, 162 b}$, S. Sushkov ${ }^{11}$,

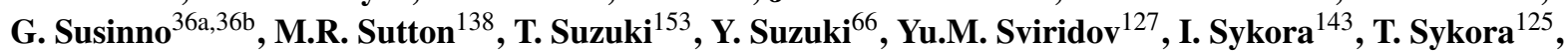
T. Szymocha ${ }^{38}$, J. Sánchez ${ }^{165}$, D. Ta ${ }^{20}$, K. Tackmann ${ }^{29}$, A. Taffard ${ }^{161}$, R. Tafirout ${ }^{157 a}$, A. Taga ${ }^{116}$, Y. Takahashi $^{101}$, H. Takai ${ }^{24}$, R. Takashima ${ }^{69}$, H. Takeda ${ }^{67}$, T. Takeshita ${ }^{139}$, M. Talby ${ }^{83}$, A. Talyshev ${ }^{106}$, M.C. Tamsett ${ }^{76}$, J. Tanaka $^{153}$, R. Tanaka $^{114}$, S. Tanaka ${ }^{130}$, S. Tanaka ${ }^{66}$, G.P. Tappern ${ }^{29}$, S. Tapprogge ${ }^{81}$, D. Tardif ${ }^{156}$, S. Tarem ${ }^{150}$, F. Tarrade ${ }^{24}$, G.F. Tartarelli ${ }^{89 a}$, P. Tas ${ }^{125}$, M. Tasevsky ${ }^{124}$, E. Tassi ${ }^{36 a, 36 b}$, M. Tatarkhanov ${ }^{14}$, C. Taylor $^{77}$, F.E. Taylor ${ }^{92}$, G.N. Taylor ${ }^{86}$, R.P. Taylor ${ }^{167}$, W. Taylor ${ }^{157 b}$, P. Teixeira-Dias ${ }^{76}$, H. Ten Kate ${ }^{29}$, P.K. Teng ${ }^{149}$,

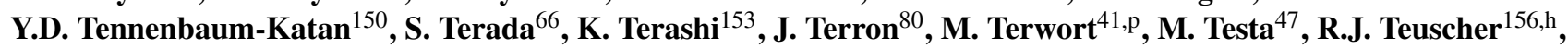
C.M. Tevlin ${ }^{82}$, J. Thadome ${ }^{172}$, R. Thananuwong ${ }^{49}$, M. Thioye ${ }^{173}$, S. Thoma ${ }^{48}$, J.P. Thomas ${ }^{17}$, T.L. Thomas ${ }^{103}$, E.N. Thompson ${ }^{84}$, P.D. Thompson ${ }^{17}$, P.D. Thompson ${ }^{156}$, R.J. Thompson ${ }^{82}$, A.S. Thompson ${ }^{53}$, E. Thomson ${ }^{119}$, R.P. Thun ${ }^{87}$, T. Tic ${ }^{124}$, V.O. Tikhomirov ${ }^{94}$, Y.A. Tikhonov ${ }^{106}$, C.J.W.P. Timmermans ${ }^{104}$, P. Tipton ${ }^{173}$, F.J. Tique

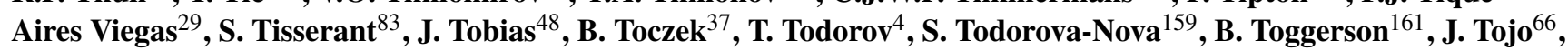

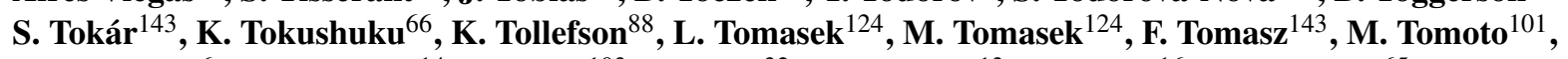
D. Tompkins ${ }^{6}$, L. Tompkins ${ }^{14}$, K. Toms ${ }^{103}$, G. Tong ${ }^{32}$, A. Tonoyan $^{13}$, C. Topfel ${ }^{16}$, N.D. Topilin ${ }^{65}$, E. Torrence ${ }^{113}$, E. Torró Pastor ${ }^{165}$, J. Toth ${ }^{83, x}$, F. Touchard ${ }^{83}$, D.R. Tovey ${ }^{138}$, S.N. Tovey ${ }^{86}$, T. Trefzger $^{171}$, L. Tremblet $^{29}$, A. Tricoli $^{29}$, I.M. Trigger ${ }^{157}$, S. Trincaz-Duvoid ${ }^{78}$, T.N. $\operatorname{Trinh}^{78}$, M.F. $\operatorname{Tripiana}^{70}$, N. $\operatorname{Triplett}^{64}$, W. $\operatorname{Trischuk}^{156}$, A. Trivedi $^{24, w}$,

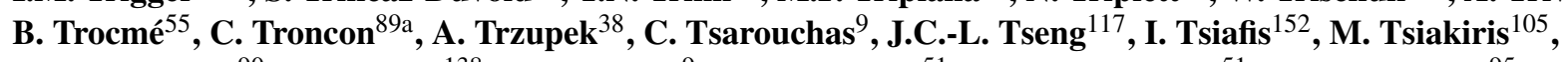
P.V. Tsiareshka ${ }^{90}$, D. Tsionou ${ }^{138}$, G. Tsipolitis ${ }^{9}$, V. Tsiskaridze ${ }^{51}$, E.G. Tskhadadze ${ }^{51}$, I.I. Tsukerman ${ }^{95}$, V. Tsulaia' ${ }^{122}$, J.-W. Tsung ${ }^{20}$, S. Tsuno ${ }^{66}$, D. Tsybychev ${ }^{146}$, M. Turala ${ }^{38}$, D. Turecek ${ }^{126}$, I. Turk Cakir ${ }^{3, \text { aa }}$,

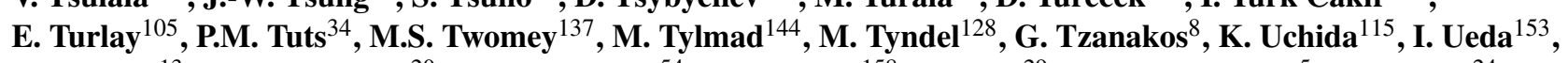
M. Ugland ${ }^{13}$, M. Uhlenbrock ${ }^{20}$, M. Uhrmacher ${ }^{54}$, F. Ukegawa ${ }^{158}$, G. Unal ${ }^{29}$, D.G. Underwood ${ }^{5}$, A. Undrus ${ }^{24}$, 
G. Unel ${ }^{161}$, Y. Unno ${ }^{66}$, D. Urbaniec ${ }^{34}$, E. Urkovsky ${ }^{151}$, P. Urquijo ${ }^{49}$, P. Urrejola ${ }^{31 \mathrm{a}}$, G. Usai ${ }^{7}$, M. Uslenghi ${ }^{118 a, 118 b}$, L. Vacavant ${ }^{83}$, V. Vacek ${ }^{126}$, B. Vachon ${ }^{85}$, S. Vahsen ${ }^{14}$, J. Valenta ${ }^{124}$, P. Valente ${ }^{131 a}$, S. Valentinetti ${ }^{19 a, 19 b}$, S. Valkar ${ }^{125}$, E. Valladolid Gallego ${ }^{165}$, S. Vallecorsa ${ }^{150}$, J.A. Valls Ferrer ${ }^{165}$, R. Van Berg ${ }^{119}$, H. van der Graaf ${ }^{105}$, E. van der Kraaij ${ }^{105}$, E. van der Poel ${ }^{105}$, D. Van Der Ster $^{29}$, N. van Eldik ${ }^{84}$, P. van Gemmeren ${ }^{5}$, Z. van Kesteren ${ }^{105}$, I. van Vulpen ${ }^{105}$, W. Vandelli ${ }^{29}$, G. Vandoni ${ }^{29}$, A. Vaniachine ${ }^{5}$, P. Vankov ${ }^{73}$, F. Vannucci ${ }^{78}$, F. Varela Rodriguez ${ }^{29}$, R. Vari $^{131 a}$, E.W. Varnes $^{6}$, D. Varouchas ${ }^{14}$, A. Vartapetian ${ }^{7}$, K.E. Varvell ${ }^{148}$, L. Vasilyeva ${ }^{94}$, V.I. Vassilakopoulos ${ }^{56}$,

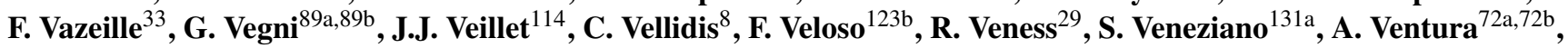
D. Ventura ${ }^{137}$, M. Venturi ${ }^{48}$, N. Venturi ${ }^{16}$, V. Vercesi ${ }^{118 a}$, M. Verducci ${ }^{171}$, W. Verkerke $^{105}$, J.C. Vermeulen ${ }^{105}$, M.C. Vetterli ${ }^{141, b}$, I. Vichou ${ }^{163}$, T. Vickey ${ }^{170}$, G.H.A. Viehhauser ${ }^{117}$, M. Villa ${ }^{19 a, 19 b}$, E.G. Villani ${ }^{128}$, M. Villaplana Perez $^{165}$, J. Villate ${ }^{123 b}$, E. Vilucchi ${ }^{47}$, M.G. Vincter ${ }^{28}$, E. Vinek $^{29}$, V.B. Vinogradov ${ }^{65}$, S. Viret $^{33}$, J. Virzi $^{14}$, A. Vitale ${ }^{19 a, 19 b}$, O.V. Vitells ${ }^{169}$, I. Vivarelli ${ }^{48}$, F. Vives Vaques ${ }^{11}$, S. Vlachos ${ }^{9}$, M. Vlasak ${ }^{126}$, N. Vlasov ${ }^{20}$, A. Vogel $^{20}$, P. Vokac ${ }^{126}$, M. Volpi ${ }^{11}$, G. Volpini ${ }^{89 a}$, H. von der Schmitt ${ }^{99}$, J. von Loeben ${ }^{99}$, H. von Radziewski ${ }^{48}$, E. von Toerne ${ }^{20}$,

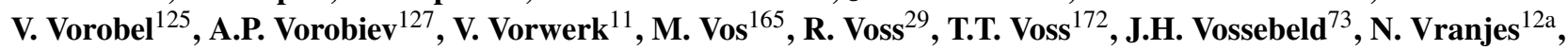
M. Vranjes Milosavljevic ${ }^{12 a}$, V. Vrba ${ }^{124}$, M. Vreeswijk ${ }^{105}$, T. Vu Anh $^{81}$, D. Vudragovic $^{12 a}$, R. Vuillermet ${ }^{29}$, I. Vukotic ${ }^{114}$, P. Wagner ${ }^{119}$, H. Wahlen ${ }^{172}$, J. Walbersloh ${ }^{42}$, J. Walder ${ }^{71}$, R. Walker ${ }^{98}$, W. Walkowiak ${ }^{140}$, R. Wall ${ }^{173}$, C. Wang ${ }^{44}$, H. Wang ${ }^{170}$, J. Wang ${ }^{55}$, J.C. Wang ${ }^{137}$, S.M. Wang ${ }^{149}$, C.P. Ward ${ }^{27}$, M. Warsinsky ${ }^{48}$, R. Wastie ${ }^{117}$, P.M. Watkins ${ }^{17}$, A.T. Watson ${ }^{17}$, M.F. Watson ${ }^{17}$, G. Watts ${ }^{137}$, S. Watts ${ }^{82}$, A.T. Waugh ${ }^{148}$, B.M. Waugh ${ }^{77}$, M. Webel ${ }^{48}$, J. Weber ${ }^{42}$, M.D. Weber ${ }^{16}$, M. Weber ${ }^{128}$, M.S. Weber ${ }^{16}$, P. Weber ${ }^{58 a}$, A.R. Weidberg ${ }^{117}$, J. Weingarten ${ }^{54}$, C. Weiser ${ }^{48}$, H. Wellenstein ${ }^{22}$, P.S. Wells ${ }^{29}$, M. Wen ${ }^{47}$, T. Wenaus ${ }^{24}$, S. Wendler ${ }^{122}$, T. Wengler ${ }^{82}$, S. Wenig ${ }^{29}$,

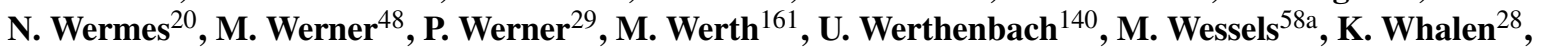
S.J. Wheeler-Ellis ${ }^{161}$, S.P. Whitaker ${ }^{21}$, A. White ${ }^{7}$, M.J. White ${ }^{27}$, S. White ${ }^{24}$, D. Whiteson ${ }^{161}$, D. Whittington ${ }^{61}$, F. Wicek ${ }^{114}$, D. Wicke ${ }^{81}$, F.J. Wickens ${ }^{128}$, W. Wiedenmann ${ }^{170}$, M. Wielers ${ }^{128}$, P. Wienemann ${ }^{20}$, C. Wiglesworth ${ }^{73}$, L.A.M. Wiik ${ }^{48}$, A. Wildauer ${ }^{165}$, M.A. Wildt ${ }^{41, p}$, I. Wilhelm ${ }^{125}$, H.G. Wilkens ${ }^{29}$, E. Williams ${ }^{34}$, H.H. Williams ${ }^{119}$, W. Willis ${ }^{34}$, S. Willocq ${ }^{84}$, J.A. Wilson ${ }^{17}$, M.G. Wilson ${ }^{142}$, A. Wilson ${ }^{87}$, I. Wingerter-Seez ${ }^{4}$, F. Winklmeier ${ }^{29}$, M. Wittgen ${ }^{142}$, M.W. Wolter ${ }^{38}$, H. Wolters ${ }^{123 b}$, B.K. Wosiek $^{38}$, J. Wotschack ${ }^{29}$, M.J. Woudstra ${ }^{84}$, K. Wraight $^{53}$,

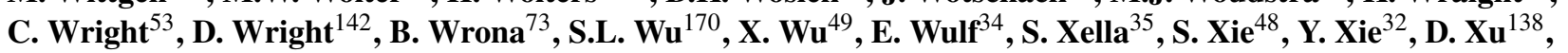
N. Xu ${ }^{170}$, M. Yamada ${ }^{158}$, A. Yamamoto ${ }^{66}$, S. Yamamoto ${ }^{153}$, T. Yamamura ${ }^{153}$, K. Yamanaka ${ }^{64}$, J. Yamaoka ${ }^{44}$,

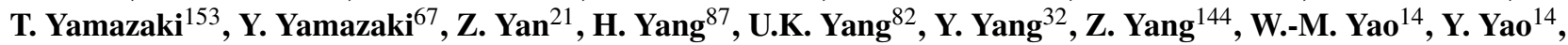

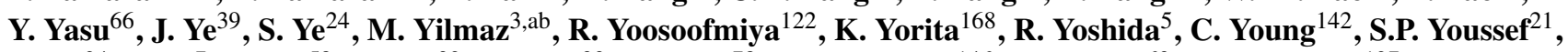
D. Yu ${ }^{24}$, J. Yu ${ }^{7}$, M. Yu ${ }^{58 c}$, X. Yu ${ }^{32}$, J. Yuan ${ }^{99}$, L. Yuan ${ }^{78}$, A. Yurkewicz ${ }^{146}$, R. Zaidan ${ }^{63}$, A.M. Zaitsev ${ }^{127}$, Z. Zajacova ${ }^{29}$, V. Zambrano ${ }^{47}$, L. Zanello ${ }^{131 \mathrm{a}, 131 \mathrm{~b}}$, P. Zarzhitsky ${ }^{39}$, A. Zaytsev ${ }^{106}$, C. Zeitnitz ${ }^{172}$, M. Zeller ${ }^{173}$, P.F. Zema ${ }^{29}$, A. Zemla ${ }^{38}$, C. Zendler ${ }^{20}$, O. Zenin ${ }^{127}$, T. Zenis ${ }^{143}$, Z. Zenonos ${ }^{121 a, 121 b}$, S. Zenz ${ }^{14}$, D. Zerwas ${ }^{114}$, G. Zevi della Porta ${ }^{57}$, Z. Zhan $^{32}$, H. Zhang ${ }^{83}$, J. Zhang ${ }^{5}$, Q. Zhang ${ }^{5}$, X. Zhang ${ }^{32}$, L. Zhao ${ }^{107}$, T. Zhao ${ }^{137}$, Z. Zhao ${ }^{32}$, A. Zhemchugov ${ }^{65}$, S. Zheng ${ }^{32}$, J. Zhong ${ }^{149, \text { ac }}$, B. Zhou ${ }^{87}$, N. Zhou ${ }^{34}$, Y. Zhou ${ }^{149}$, C.G. Zhu ${ }^{32}$, H. Zhu ${ }^{41}$, Y. Zhu ${ }^{170}$, X. Zhuang ${ }^{98}$, V. Zhuravlov ${ }^{99}$, R. Zimmermann ${ }^{20}$, S. Zimmermann ${ }^{20}$, S. Zimmermann ${ }^{48}$, M. Ziolkowski ${ }^{140}$, R. Zitoun ${ }^{4}$, L. Živković ${ }^{34}$, V.V. Zmouchko ${ }^{127, *}$, G. Zobernig ${ }^{170}$, A. Zoccoli ${ }^{19 a, 19 b}$, M. zur Nedden ${ }^{15}$, V. Zutshi ${ }^{5}$

${ }^{\star}$ CERN, 1211 Geneva 23, Switzerland

${ }^{1}$ University at Albany, 1400 Washington Ave, Albany, NY 12222, United States of America

${ }^{2}$ University of Alberta, Department of Physics, Centre for Particle Physics, Edmonton, AB T6G 2G7, Canada

${ }^{3}$ Ankara University, Faculty of Sciences, Department of Physics, TR 061000 Tandogan, Ankara, Turkey

${ }^{4}$ LAPP, Université de Savoie, CNRS/IN2P3, Annecy-le-Vieux, France

${ }^{5}$ Argonne National Laboratory, High Energy Physics Division, 9700 S. Cass Avenue, Argonne IL 60439, United States of America

${ }^{6}$ University of Arizona, Department of Physics, Tucson, AZ 85721, United States of America

${ }^{7}$ The University of Texas at Arlington, Department of Physics, Box 19059, Arlington, TX 76019, United States of America

${ }^{8}$ University of Athens, Nuclear \& Particle Physics, Department of Physics, Panepistimiopouli, Zografou, GR 15771 Athens, Greece

${ }^{9}$ National Technical University of Athens, Physics Department, 9-Iroon Polytechniou, GR 15780 Zografou, Greece

${ }^{10}$ Institute of Physics, Azerbaijan Academy of Sciences, H. Javid Avenue 33, AZ 143 Baku, Azerbaijan

${ }^{11}$ Institut de Física d'Altes Energies, IFAE, Edifici Cn, Universitat Autònoma de Barcelona, ES-08193 Bellaterra (Barcelona), Spain

12(a) University of Belgrade, Institute of Physics, P.O. Box 57, 11001 Belgrade; Vinca Institute of Nuclear Sciences ${ }^{(\mathrm{b})}$, Mihajla Petrovica Alasa 12-14, 11001 Belgrade, Serbia

${ }^{13}$ University of Bergen, Department for Physics and Technology, Allegaten 55, NO-5007 Bergen, Norway

${ }^{14}$ Lawrence Berkeley National Laboratory and University of California, Physics Division, MS50B-6227, 1 Cyclotron Road, Berkeley, CA 94720, United States of America

${ }^{15}$ Humboldt University, Institute of Physics, Berlin, Newtonstr. 15, D-12489 Berlin, Germany

${ }^{16}$ University of Bern, Albert Einstein Center for Fundamental Physics, Laboratory for High Energy Physics, Sidlerstrasse 5, CH-3012 Bern, Switzerland 
${ }^{17}$ University of Birmingham, School of Physics and Astronomy, Edgbaston, Birmingham B15 2TT, United Kingdom

${ }^{18}$ Bogazici University, Faculty of Sciences, Department of Physics, TR-80815 Bebek-Istanbul, Turkey

${ }^{19}$ INFN Sezione di Bologna ${ }^{(\mathrm{a})}$; Università di Bologna, Dipartimento di Fisica ${ }^{(\mathrm{b})}$, viale C. Berti Pichat, 6/2, IT-40127 Bologna, Italy

${ }^{20}$ University of Bonn, Physikalisches Institut, Nussallee 12, D-53115 Bonn, Germany

${ }^{21}$ Boston University, Department of Physics, 590 Commonwealth Avenue, Boston, MA 02215, United States of America

${ }^{22}$ Brandeis University, Department of Physics, MS057, 415 South Street, Waltham, MA 02454, United States of America

${ }^{23}$ Universidade Federal do Rio De Janeiro, Instituto de Fisica ${ }^{(a)}$, Caixa Postal 68528, Ilha do Fundao, BR-21945-970 Rio de Janeiro;

(b) Universidade de Sao Paulo, Instituto de Fisica, R.do Matao Trav. R.187, Sao Paulo-SP, 05508-900, Brazil

${ }^{24}$ Brookhaven National Laboratory, Physics Department, Bldg. 510A, Upton, NY 11973, United States of America

${ }^{25}$ National Institute of Physics and Nuclear Engineering ${ }^{(a)}$, Bucharest-Magurele, Str. Atomistilor 407, P.O. Box MG-6, R-077125, Romania;

(b) University Politehnica Bucharest, Rectorat-AN 001, 313 Splaiul Independentei, sector 6, 060042 Bucuresti; (c) West University in Timisoara, Bd. Vasile Parvan 4, Timisoara, Romania

${ }^{26}$ Universidad de Buenos Aires, FCEyN, Dto. Fisica, Pab I-C. Universitaria, 1428 Buenos Aires, Argentina

${ }^{27}$ University of Cambridge, Cavendish Laboratory, J J Thomson Avenue, Cambridge CB3 OHE, United Kingdom

${ }^{28}$ Carleton University, Department of Physics, 1125 Colonel By Drive, Ottawa ON K1S 5B6, Canada

${ }^{29}$ CERN, CH-1211 Geneva 23, Switzerland

${ }^{30}$ University of Chicago, Enrico Fermi Institute, 5640 S. Ellis Avenue, Chicago, IL 60637, United States of America

${ }^{31}$ Pontificia Universidad Católica de Chile, Facultad de Fisica, Departamento de Fisica ${ }^{(a)}$, Avda. Vicuna Mackenna 4860, San Joaquin, Santiago; Universidad Técnica Federico Santa María, Departamento de Física ${ }^{(b)}$, Avda. Espãna 1680, Casilla 110-V, Valparaíso, Chile

${ }^{32}$ Institute of HEP, Chinese Academy of Sciences, P.O. Box 918, CN-100049 Beijing; USTC, Department of Modern Physics, Hefei, CN-230026 Anhui; Nanjing University, Department of Physics, CN-210093 Nanjing; Shandong University, HEP Group, CN-250100 Shadong, China

${ }^{33}$ Laboratoire de Physique Corpusculaire, CNRS-IN2P3, Université Blaise Pascal, FR-63177 Aubiere Cedex, France

${ }^{34}$ Columbia University, Nevis Laboratory, 136 So. Broadway, Irvington, NY 10533, United States of America

${ }^{35}$ University of Copenhagen, Niels Bohr Institute, Blegdamsvej 17, DK-2100 Kobenhavn 0, Denmark

${ }^{36}$ INFN Gruppo Collegato di Cosenza ${ }^{(a)}$; Università della Calabria, Dipartimento di Fisica ${ }^{(b)}$, IT-87036 Arcavacata di Rende, Italy

${ }^{37}$ Faculty of Physics and Applied Computer Science of the AGH-University of Science and Technology (FPACS, AGH-UST), al. Mickiewicza 30, PL-30059 Cracow, Poland

${ }^{38}$ The Henryk Niewodniczanski Institute of Nuclear Physics, Polish Academy of Sciences, ul. Radzikowskiego 152, PL-31342 Krakow, Poland

${ }^{39}$ Southern Methodist University, Physics Department, 106 Fondren Science Building, Dallas, TX 75275-0175, United States of America

${ }^{40}$ University of Texas at Dallas, 800 West Campbell Road, Richardson, TX 75080-3021, United States of America

${ }^{41}$ DESY, Notkestr. 85, D-22603 Hamburg, Germany and Platanenallee 6, D-15738 Zeuthen, Germany

${ }^{42}$ TU Dortmund, Experimentelle Physik IV, DE-44221 Dortmund, Germany

${ }^{43}$ Technical University Dresden, Institut fuer Kern- und Teilchenphysik, Zellescher Weg 19, D-01069 Dresden, Germany

${ }^{44}$ Duke University, Department of Physics, Durham, NC 27708, United States of America

${ }^{45}$ University of Edinburgh, School of Physics \& Astronomy, James Clerk Maxwell Building, The Kings Buildings, Mayfield Road, Edinburgh EH9 3JZ, United Kingdom

${ }^{46}$ Fachhochschule Wiener Neustadt; Johannes Gutenbergstrasse 3 AT-2700 Wiener Neustadt, Austria

${ }^{47}$ INFN Laboratori Nazionali di Frascati, via Enrico Fermi 40, IT-00044 Frascati, Italy

${ }^{48}$ Albert-Ludwigs-Universität, Fakultät für Mathematik und Physik, Hermann-Herder Str. 3, D-79104 Freiburg i.Br., Germany

${ }^{49}$ Université de Genève, Section de Physique, 24 rue Ernest Ansermet, CH-1211 Geneve 4, Switzerland

${ }^{50}$ INFN Sezione di Genova $^{(a)}$; Università di Genova, Dipartimento di Fisica ${ }^{(b)}$, via Dodecaneso 33, IT-16146 Genova, Italy

${ }^{51}$ Institute of Physics of the Georgian Academy of Sciences, 6 Tamarashvili St., GE-380077 Tbilisi; Tbilisi State University, HEP Institute, University St. 9, GE-380086 Tbilisi, Georgia

${ }^{52}$ Justus-Liebig-Universitaet Giessen, II Physikalisches Institut, Heinrich-Buff Ring 16, D-35392 Giessen, Germany

${ }^{53}$ University of Glasgow, Department of Physics and Astronomy, Glasgow G12 8QQ, United Kingdom

${ }^{54}$ Georg-August-Universitat, II. Physikalisches Institut, Friedrich-Hund Platz 1, D-37077 Goettingen, Germany

${ }^{55}$ Laboratoire de Physique Subatomique et de Cosmologie, CNRS/IN2P3, Université Joseph Fourier, INPG, 53 avenue des Martyrs, FR-38026 Grenoble Cedex, France

${ }^{56}$ Hampton University, Department of Physics, Hampton, VA 23668, United States of America

${ }^{57}$ Harvard University, Laboratory for Particle Physics and Cosmology, 18 Hammond Street, Cambridge, MA 02138, United States of America

${ }^{58}$ Ruprecht-Karls-Universitaet Heidelberg, Kirchhoff-Institut fuer Physik ${ }^{(a)}$, Im Neuenheimer Feld 227, D-69120 Heidelberg; ${ }^{\text {(b) }}$ Physikalisches Institut, Philosophenweg 12, D-69120 Heidelberg; ZITI Ruprecht-Karls-University Heidelberg ${ }^{(\mathrm{c})}$, Lehrstuhl fuer Informatik V, B6, 23-29, DE-68131 Mannheim, Germany

${ }^{59}$ Hiroshima University, Faculty of Science, 1-3-1 Kagamiyama, Higashihiroshima-shi, JP-Hiroshima 739-8526, Japan

${ }^{60}$ Hiroshima Institute of Technology, Faculty of Applied Information Science, 2-1-1 Miyake Saeki-ku, Hiroshima-shi, JP-Hiroshima 731-5193, Japan

${ }^{61}$ Indiana University, Department of Physics, Swain Hall West 117, Bloomington, IN 47405-7105, United States of America

${ }^{62}$ Institut fuer Astro- und Teilchenphysik, Technikerstrasse 25, A-6020 Innsbruck, Austria

${ }^{63}$ University of Iowa, 203 Van Allen Hall, Iowa City, IA 52242-1479, United States of America

${ }^{64}$ Iowa State University, Department of Physics and Astronomy, Ames High Energy Physics Group, Ames, IA 50011-3160, United States of America

${ }^{65}$ Joint Institute for Nuclear Research, JINR Dubna, RU-141 980 Moscow Region, Russia

${ }^{66}$ KEK, High Energy Accelerator Research Organization, 1-1 Oho, Tsukuba-shi, Ibaraki-ken 305-0801, Japan

${ }^{67}$ Kobe University, Graduate School of Science, 1-1 Rokkodai-cho, Nada-ku, JP Kobe 657-8501, Japan

${ }^{68}$ Kyoto University, Faculty of Science, Oiwake-cho, Kitashirakawa, Sakyou-ku, Kyoto-shi, JP-Kyoto 606-8502, Japan 
${ }^{69}$ Kyoto University of Education, 1 Fukakusa, Fujimori, fushimi-ku, Kyoto-shi, JP-Kyoto 612-8522, Japan

${ }^{70}$ Universidad Nacional de La Plata, FCE, Departamento de Física, IFLP (CONICET-UNLP), C.C. 67, 1900 La Plata, Argentina

${ }^{71}$ Lancaster University, Physics Department, Lancaster LA1 4YB, United Kingdom

${ }^{72}$ INFN Sezione di Lecce ${ }^{(a)}$; Università del Salento, Dipartimento di Fisica ${ }^{(b)}$ Via Arnesano IT-73100 Lecce, Italy

${ }^{73}$ University of Liverpool, Oliver Lodge Laboratory, P.O. Box 147, Oxford Street, Liverpool L69 3BX, United Kingdom

${ }^{74}$ Jožef Stefan Institute and University of Ljubljana, Department of Physics, SI-1000 Ljubljana, Slovenia

${ }^{75}$ Queen Mary University of London, Department of Physics, Mile End Road, London E1 4NS, United Kingdom

${ }^{76}$ Royal Holloway, University of London, Department of Physics, Egham Hill, Egham, Surrey TW20 0EX, United Kingdom

${ }^{77}$ University College London, Department of Physics and Astronomy, Gower Street, London WC1E 6BT, United Kingdom

${ }^{78}$ Laboratoire de Physique Nucléaire et de Hautes Energies, Université Pierre et Marie Curie (Paris 6), Université Denis Diderot (Paris-7), CNRS/IN2P3, Tour 33, 4 place Jussieu, FR-75252 Paris Cedex 05, France

${ }^{79}$ Lunds universitet, Naturvetenskapliga fakulteten, Fysiska institutionen, Box 118, SE-221 00 Lund, Sweden

${ }^{80}$ Universidad Autonoma de Madrid, Facultad de Ciencias, Departamento de Fisica Teorica, ES-28049 Madrid, Spain

${ }^{81}$ Universitaet Mainz, Institut fuer Physik, Staudinger Weg 7, DE-55099 Mainz, Germany

${ }^{82}$ University of Manchester, School of Physics and Astronomy, Manchester M13 9PL, United Kingdom

${ }^{83}$ CPPM, Aix-Marseille Université, CNRS/IN2P3, Marseille, France

${ }^{84}$ University of Massachusetts, Department of Physics, 710 North Pleasant Street, Amherst, MA 01003, United States of America

${ }^{85}$ McGill University, High Energy Physics Group, 3600 University Street, Montreal, Quebec H3A 2T8, Canada

${ }^{86}$ University of Melbourne, School of Physics, AU-Parkville, Victoria 3010, Australia

${ }^{87}$ The University of Michigan, Department of Physics, 2477 Randall Laboratory, 500 East University, Ann Arbor, MI 48109-1120, United States of America

${ }^{88}$ Michigan State University, Department of Physics and Astronomy, High Energy Physics Group, East Lansing, MI 48824-2320, United States of America

${ }^{89}$ INFN Sezione di Milano $^{(\mathrm{a})}$; Università di Milano, Dipartimento di Fisica ${ }^{(\mathrm{b})}$, via Celoria 16, IT-20133 Milano, Italy

${ }^{90}$ B.I. Stepanov Institute of Physics, National Academy of Sciences of Belarus, Independence Avenue 68, Minsk 220072, Republic of Belarus

${ }^{91}$ National Scientific \& Educational Centre for Particle \& High Energy Physics, NC PHEP BSU, M. Bogdanovich St. 153, Minsk 220040, Republic of Belarus

${ }^{92}$ Massachusetts Institute of Technology, Department of Physics, Room 24-516, Cambridge, MA 02139, United States of America

${ }^{93}$ University of Montreal, Group of Particle Physics, C.P. 6128, Succursale Centre-Ville, Montreal, Quebec, H3C 3J7, Canada

${ }^{94}$ P.N. Lebedev Institute of Physics, Academy of Sciences, Leninsky pr. 53, RU-117 924 Moscow, Russia

${ }^{95}$ Institute for Theoretical and Experimental Physics (ITEP), B. Cheremushkinskaya ul. 25, RU 117218 Moscow, Russia

${ }^{96}$ Moscow Engineering \& Physics Institute (MEPhI), Kashirskoe Shosse 31, RU-115409 Moscow, Russia

${ }^{97}$ Lomonosov Moscow State University Skobeltsyn Institute of Nuclear Physics (MSU SINP), 1(2), Leninskie gory, GSP-1, Moscow 119991 Russian Federation, Russia

${ }^{98}$ Ludwig-Maximilians-Universität München, Fakultät für Physik, Am Coulombwall 1, DE-85748 Garching, Germany

${ }^{99}$ Max-Planck-Institut für Physik, (Werner-Heisenberg-Institut), Föhringer Ring 6, 80805 München, Germany

${ }^{100}$ Nagasaki Institute of Applied Science, 536 Aba-machi, JP Nagasaki 851-0193, Japan

${ }^{101}$ Nagoya University, Graduate School of Science, Furo-Cho, Chikusa-ku, Nagoya, 464-8602, Japan

${ }^{102}$ INFN Sezione di Napoli ${ }^{(a)}$; Università di Napoli, Dipartimento di Scienze Fisiche ${ }^{(b)}$, Complesso Universitario di Monte Sant'Angelo, via Cinthia, IT-80126 Napoli, Italy

${ }^{103}$ University of New Mexico, Department of Physics and Astronomy, MSC07 4220, Albuquerque, NM 87131 USA, United States of America

${ }^{104}$ Radboud University Nijmegen/NIKHEF, Department of Experimental High Energy Physics, Toernooiveld 1, NL-6525 ED Nijmegen, Netherlands

${ }^{105}$ Nikhef National Institute for Subatomic Physics, and University of Amsterdam, Science Park 105, 1098 XG Amsterdam, Netherlands

${ }^{106}$ Budker Institute of Nuclear Physics (BINP), RU-Novosibirsk 630 090, Russia

${ }^{107}$ New York University, Department of Physics, 4 Washington Place, New York NY 10003, USA, United States of America

${ }^{108}$ Ohio State University, 191 West Woodruff Ave, Columbus, OH 43210-1117, United States of America

${ }^{109}$ Okayama University, Faculty of Science, Tsushimanaka 3-1-1, Okayama 700-8530, Japan

${ }^{110}$ University of Oklahoma, Homer L. Dodge Department of Physics and Astronomy, 440 West Brooks, Room 100, Norman, OK 73019-0225, United States of America

${ }^{111}$ Oklahoma State University, Department of Physics, 145 Physical Sciences Building, Stillwater, OK 74078-3072, United States of America

${ }^{112}$ Palacký University, 17.listopadu 50a, 77207 Olomouc, Czech Republic

${ }^{113}$ University of Oregon, Center for High Energy Physics, Eugene, OR 97403-1274, United States of America

${ }^{114}$ LAL, Univ. Paris-Sud, IN2P3/CNRS, Orsay, France

${ }^{115}$ Osaka University, Graduate School of Science, Machikaneyama-machi 1-1, Toyonaka, Osaka 560-0043, Japan

${ }^{116}$ University of Oslo, Department of Physics, P.O. Box 1048, Blindern, NO-0316 Oslo 3, Norway

${ }^{117}$ Oxford University, Department of Physics, Denys Wilkinson Building, Keble Road, Oxford OX1 3RH, United Kingdom

${ }^{118}$ INFN Sezione di Pavia $^{(\mathrm{a})}$; Università di Pavia, Dipartimento di Fisica Nucleare e Teorica ${ }^{(\mathrm{b})}$, Via Bassi 6, IT-27100 Pavia, Italy

${ }^{119}$ University of Pennsylvania, Department of Physics, High Energy Physics Group, 209 S. 33rd Street, Philadelphia, PA 19104, United States of America

${ }^{120}$ Petersburg Nuclear Physics Institute, RU-188 300 Gatchina, Russia

${ }^{121}$ INFN Sezione di Pisa ${ }^{(a)}$; Università di Pisa, Dipartimento di Fisica E. Fermi ${ }^{(\mathrm{b})}$, Largo B. Pontecorvo 3, IT-56127 Pisa, Italy

${ }^{122}$ University of Pittsburgh, Department of Physics and Astronomy, 3941 O'Hara Street, Pittsburgh, PA 15260, United States of America

123(a) Universidad de Granada, Departamento de Fisica Teorica y del Cosmos and CAFPE, E-18071 Granada; Laboratorio de Instrumentacao e Fisica Experimental de Particulas-LIP ${ }^{(b)}$, Avenida Elias Garcia 14-1, PT-1000-149 Lisboa, Portugal 
${ }^{124}$ Institute of Physics, Academy of Sciences of the Czech Republic, Na Slovance 2, CZ-18221 Praha 8, Czech Republic

${ }^{125}$ Charles University in Prague, Faculty of Mathematics and Physics, Institute of Particle and Nuclear Physics, V Holesovickach 2, CZ-18000 Praha 8, Czech Republic

${ }^{126}$ Czech Technical University in Prague, Zikova 4, CZ-166 35 Praha 6, Czech Republic

${ }^{127}$ State Research Center Institute for High Energy Physics, Moscow Region, 142281, Protvino, Pobeda street, 1, Russia

${ }^{128}$ Rutherford Appleton Laboratory, Science and Technology Facilities Council, Harwell Science and Innovation Campus, Didcot OX11 0QX, United Kingdom

${ }^{129}$ University of Regina, Physics Department, Canada

${ }^{130}$ Ritsumeikan University, Noji Higashi 1 chome 1-1, JP-Kusatsu, Shiga 525-8577, Japan

${ }^{131}$ INFN Sezione di Roma I ${ }^{(a)}$; Università La Sapienza, Dipartimento di Fisica ${ }^{(b)}$, Piazzale A. Moro 2, IT- 00185 Roma, Italy

${ }^{132}$ INFN Sezione di Roma Tor Vergata ${ }^{(a)}$; Università di Roma Tor Vergata, Dipartimento di Fisica ${ }^{(b)}$, via della Ricerca Scientifica, IT-00133 Roma, Italy

${ }^{133}$ INFN Sezione di Roma Tre ${ }^{(a)}$; Università Roma Tre, Dipartimento di Fisica ${ }^{(b)}$, via della Vasca Navale 84, IT-00146 Roma, Italy

${ }^{134}$ Université Hassan II, Faculté des Sciences Ain Chock $^{(a)}$, B.P. 5366, MA-Casablanca; Centre National de l'Energie des Sciences Techniques Nucleaires $\left(\right.$ CNESTEN) $^{(b)}$, B.P. 1382 R.P. 10001 Rabat 10001; Université Mohamed Premier ${ }^{(c)}$, LPTPM, Faculté des Sciences, B.P.717. Bd. Mohamed VI, 60000, Oujda; Université Mohammed V, Faculté des Sciences ${ }^{(\mathrm{d})}$, LPNR, BP 1014, 10000 Rabat, Morocco

${ }^{135}$ CEA, DSM/IRFU, Centre d'Etudes de Saclay, FR-91191 Gif-sur-Yvette, France

${ }^{136}$ University of California Santa Cruz, Santa Cruz Institute for Particle Physics (SCIPP), Santa Cruz, CA 95064, United States of America

${ }^{137}$ University of Washington, Seattle, Department of Physics, Box 351560, Seattle, WA 98195-1560, United States of America

${ }^{138}$ University of Sheffield, Department of Physics \& Astronomy, Hounsfield Road, Sheffield S3 7RH, United Kingdom

${ }^{139}$ Shinshu University, Department of Physics, Faculty of Science, 3-1-1 Asahi, Matsumoto-shi, JP-Nagano 390-8621, Japan

${ }^{140}$ Universitaet Siegen, Fachbereich Physik, D 57068 Siegen, Germany

${ }^{141}$ Simon Fraser University, Department of Physics, 8888 University Drive, CA-Burnaby, BC V5A 1S6, Canada

${ }^{142}$ SLAC National Accelerator Laboratory, Stanford, California 94309, United States of America

${ }^{143}$ Comenius University, Faculty of Mathematics, Physics \& Informatics, Mlynska dolina F2, SK-84248 Bratislava; Institute of Experimental Physics of the Slovak Academy of Sciences, Dept. of Subnuclear Physics, Watsonova 47, SK-04353 Kosice, Slovak Republic

${ }^{144}$ Stockholm University, Department of Physics, AlbaNova, SE-106 91 Stockholm, Sweden

${ }^{145}$ Royal Institute of Technology (KTH), Physics Department, SE-106 91 Stockholm, Sweden

${ }^{146}$ Stony Brook University, Department of Physics and Astronomy, Nicolls Road, Stony Brook, NY 11794-3800, United States of America

${ }^{147}$ University of Sussex, Department of Physics and Astronomy Pevensey 2 Building, Falmer, Brighton BN1 9QH, United Kingdom

${ }^{148}$ University of Sydney, School of Physics, AU-Sydney NSW 2006, Australia

${ }^{149}$ Insitute of Physics, Academia Sinica, TW-Taipei 11529, Taiwan

${ }^{150}$ Technion, Israel Inst. of Technology, Department of Physics, Technion City, IL-Haifa 32000, Israel

${ }^{151}$ Tel Aviv University, Raymond and Beverly Sackler School of Physics and Astronomy, Ramat Aviv, IL-Tel Aviv 69978, Israel

${ }^{152}$ Aristotle University of Thessaloniki, Faculty of Science, Department of Physics, Division of Nuclear \& Particle Physics, University Campus, GR-54124, Thessaloniki, Greece

${ }^{153}$ The University of Tokyo, International Center for Elementary Particle Physics and Department of Physics, 7-3-1 Hongo, Bunkyo-ku, JP-Tokyo 113-0033, Japan

${ }^{154}$ Tokyo Metropolitan University, Graduate School of Science and Technology, 1-1 Minami-Osawa, Hachioji, Tokyo 192-0397, Japan

${ }^{155}$ Tokyo Institute of Technology, 2-12-1-H-34 O-Okayama, Meguro, Tokyo 152-8551, Japan

${ }^{156}$ University of Toronto, Department of Physics, 60 Saint George Street, Toronto M5S 1A7, Ontario, Canada

${ }^{157}$ TRIUMF $^{(\mathrm{a})}$, 4004 Wesbrook Mall, Vancouver, B.C. V6T 2A3; (b) York University, Department of Physics and Astronomy, 4700 Keele St., Toronto, Ontario, M3J 1P3, Canada

${ }^{158}$ University of Tsukuba, Institute of Pure and Applied Sciences, 1-1-1 Tennoudai, Tsukuba-shi, JP-Ibaraki 305-8571, Japan

${ }^{159}$ Tufts University, Science \& Technology Center, 4 Colby Street, Medford, MA 02155, United States of America

${ }^{160}$ Universidad Antonio Narino, Centro de Investigaciones, Cra 3 Este No. 47A-15, Bogota, Colombia

${ }^{161}$ University of California, Irvine, Department of Physics \& Astronomy, CA 92697-4575, United States of America

${ }^{162}$ INFN Gruppo Collegato di Udine ${ }^{(\mathrm{a})}$; ICTP $^{(\mathrm{b})}$, Strada Costiera 11, IT-34014, Trieste; Università di Udine, Dipartimento di Fisica ${ }^{(\mathrm{c})}$, via delle Scienze 208, IT-33100 Udine, Italy

${ }^{163}$ University of Illinois, Department of Physics, 1110 West Green Street, Urbana, Illinois 61801, United States of America

${ }^{164}$ University of Uppsala, Department of Physics and Astronomy, P.O. Box 516, SE -751 20 Uppsala, Sweden

${ }^{165}$ Instituto de Física Corpuscular (IFIC) Centro Mixto UVEG-CSIC, Apdo. 22085 ES-46071 Valencia, Dept. Física At. Mol. y Nuclear; Univ. of Valencia, and Instituto de Microelectrónica de Barcelona (IMB-CNM-CSIC) 08193 Bellaterra Barcelona, Spain

${ }^{166}$ University of British Columbia, Department of Physics, 6224 Agricultural Road, CA-Vancouver, B.C. V6T 1Z1, Canada

${ }^{167}$ University of Victoria, Department of Physics and Astronomy, P.O. Box 3055, Victoria B.C., V8W 3P6, Canada

${ }^{168}$ Waseda University, WISE, 3-4-1 Okubo, Shinjuku-ku, Tokyo, 169-8555, Japan

${ }^{169}$ The Weizmann Institute of Science, Department of Particle Physics, P.O. Box 26, IL-76100 Rehovot, Israel

${ }^{170}$ University of Wisconsin, Department of Physics, 1150 University Avenue, WI 53706 Madison, Wisconsin, United States of America

${ }^{171}$ Julius-Maximilians-University of Würzburg, Physikalisches Institute, Am Hubland, 97074 Wuerzburg, Germany

${ }^{172}$ Bergische Universitaet, Fachbereich C, Physik, Postfach 100127, Gauss-Strasse 20, D-42097 Wuppertal, Germany

${ }^{173}$ Yale University, Department of Physics, P.O. Box 208121, New Haven CT, 06520-8121, United States of America

${ }^{174}$ Yerevan Physics Institute, Alikhanian Brothers Street 2, AM-375036 Yerevan, Armenia

${ }^{175}$ ATLAS-Canada Tier-1 Data Centre 4004 Wesbrook Mall, Vancouver, BC, V6T 2A3, Canada

${ }^{176}$ GridKA Tier-1 FZK, Forschungszentrum Karlsruhe GmbH, Steinbuch Centre for Computing (SCC), Hermann-von-Helmholtz-Platz 1, 76344 Eggenstein-Leopoldshafen, Germany 
${ }^{177}$ Port d'Informacio Cientifica (PIC), Universitat Autonoma de Barcelona (UAB), Edifici D, E-08193 Bellaterra, Spain

${ }^{178}$ Centre de Calcul CNRS/IN2P3, Domaine scientifique de la Doua, 27 bd du 11 Novembre 1918, 69622 Villeurbanne Cedex, France

${ }^{179}$ INFN-CNAF, Viale Berti Pichat 6/2, 40127 Bologna, Italy

${ }^{180}$ Nordic Data Grid Facility, NORDUnet A/S, Kastruplundgade 22, 1, DK-2770 Kastrup, Denmark

${ }^{181}$ SARA Reken- en Netwerkdiensten, Science Park 121, 1098 XG Amsterdam, Netherlands

${ }^{182}$ Academia Sinica Grid Computing, Institute of Physics, Academia Sinica, No. 128, Sec. 2, Academia Rd., Nankang, Taipei, Taiwan 11529, Taiwan

${ }^{183}$ UK-T1-RAL Tier-1, Rutherford Appleton Laboratory, Science and Technology Facilities Council, Harwell Science and Innovation Campus, Didcot OX11 0QX, United Kingdom

${ }^{184}$ RHIC and ATLAS Computing Facility, Physics Department, Building 510, Brookhaven National Laboratory, Upton, New York 11973, United States of America

${ }^{a}$ Also at CPPM, Marseille, France.

${ }^{\mathrm{b}}$ Also at TRIUMF, 4004 Wesbrook Mall, Vancouver, B.C. V6T 2A3, Canada.

${ }^{\mathrm{c}}$ Also at Gaziantep University, Turkey.

${ }^{\mathrm{d}}$ Also at Faculty of Physics and Applied Computer Science of the AGH-University of Science and Technology (FPACS, AGH-UST), al. Mickiewicza 30, PL-30059 Cracow, Poland.

eAlso at Institute for Particle Phenomenology, Ogden Centre for Fundamental Physics, Department of Physics, University of Durham, Science Laboratories, South Rd, Durham DH1 3LE, United Kingdom.

${ }^{\mathrm{f}}$ Currently at Dogus University, Kadik.

${ }^{\mathrm{g}}$ Also at Università di Napoli Parthenope, via A. Acton 38, IT-80133 Napoli, Italy.

${ }^{\mathrm{h}}$ Also at Institute of Particle Physics (IPP), Canada.

${ }^{\mathrm{i}}$ Louisiana Tech University, 305 Wisteria Street, P.O. Box 3178, Ruston, LA 71272, United States of America.

${ }^{\mathrm{j}}$ Currently at Dumlupinar University, Kutahya, Turkey.

${ }^{\mathrm{k}}$ Currently at Department of Physics, University of Helsinki, P.O. Box 64, FI-00014, Finland.

${ }^{1}$ At Department of Physics, California State University, Fresno, 2345 E. San Ramon Avenue, Fresno, CA 93740-8031, United States of America.

${ }^{\mathrm{n}}$ Also at California Institute of Technology, Physics Department, Pasadena, CA 91125, United States of America.

${ }^{\circ}$ Also at University of Montreal, Canada.

pAlso at Institut für Experimentalphysik, Universität Hamburg, Luruper Chaussee 149, 22761 Hamburg, Germany.

${ }^{\mathrm{q}}$ Also at Petersburg Nuclear Physics Institute, RU-188 300 Gatchina, Russia.

${ }^{\mathrm{r}}$ Also at School of Physics and Engineering, Sun Yat-sen University, Taiwan.

${ }^{\mathrm{s}}$ Also at School of Physics, Shandong University, Jinan, China.

${ }^{\mathrm{t}}$ Also at Rutherford Appleton Laboratory, Science and Technology Facilities Council, Harwell Science and Innovation Campus, Didcot OX11, United Kingdom.

uAlso at school of physics, Shandong university, Jinan.

${ }^{v}$ Also at Rutherford Appleton Laboratory, Science and Technology Facilities Council, Harwell Science and Innovation Campus, Didcot OX11 0QX, United Kingdom.

${ }^{\mathrm{w}}$ University of South Carolina, Dept. of Physics and Astronomy, 700 S. Main St, Columbia, SC 29208, United States of America.

${ }^{\mathrm{x}}$ Also at KFKI Research Institute for Particle and Nuclear Physics, Budapest, Hungary.

${ }^{\mathrm{y}}$ Also at Institute of Physics, Jagiellonian University, Cracow, Poland.

${ }^{\mathrm{z} C u r r e n t l y ~ a t ~ T O B B ~ U n i v e r s i t y, ~ A n k a r a, ~ T u r k e y . ~}$

${ }^{\text {aa }}$ Currently at TAEA, Ankara, Turkey.

${ }^{\mathrm{ab}}$ Currently at Gazi University, Ankara, Turkey.

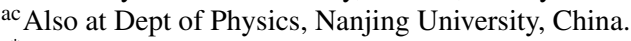

*Deceased.

Received: 22 February 2010 / Revised: 7 June 2010 / Published online: 23 October 2010

(c) CERN for the benefit of the ATLAS collaboration 2010. This article is published with open access at Springerlink.com

\begin{abstract}
The ionization signals in the liquid argon of the ATLAS electromagnetic calorimeter are studied in detail using cosmic muons. In particular, the drift time of the ionization electrons is measured and used to assess the intrinsic uniformity of the calorimeter gaps and estimate its impact on the constant term of the energy resolution. The drift times of electrons in the cells of the second layer of the calorimeter are uniform at the level of $1.3 \%$ in the barrel and $2.8 \%$ in the endcaps. This leads to an estimated contribution to the constant term of $\left(0.29_{-0.04}^{+0.05}\right) \%$ in the barrel and $\left(0.54_{-0.04}^{+0.06}\right) \%$ in the endcaps. The same data are used to measure the drift ve-
\end{abstract}

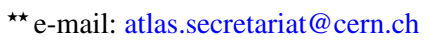

locity of ionization electrons in liquid argon, which is found to be $4.61 \pm 0.07 \mathrm{~mm} / \mu$ s at $88.5 \mathrm{~K}$ and $1 \mathrm{kV} / \mathrm{mm}$.

\section{Introduction}

The ATLAS liquid argon (LAr) calorimeter [1] is composed of sampling detectors with full azimuthal ${ }^{1}$ symmetry and is housed in one barrel and two endcap cryostats.

\footnotetext{
${ }^{1}$ The azimuthal angle $\phi$ is measured in the plane transverse to the beam axis. Positive $\phi$ is in the up direction. The pseudorapidity is defined as $\eta=-\ln (\tan (\theta / 2))$, where $\theta$ is the polar angle from the beam axis. Positive $\eta$ is for the proton beam circulating anticlockwise.
} 
A highly granular electromagnetic (EM) calorimeter with accordion-shaped electrodes and lead absorbers covers the pseudorapidity range $|\eta|<3.2$, and contains a barrel part $(|\eta|<1.475)$ [2] made of two half-barrels joined at $\eta=0$ and two endcap parts $(1.375<|\eta|<3.2)$ [3]. Each section is segmented in depth in three layers (denoted as layer 1, 2, 3). For $|\eta|<1.8$, a presampler (PS) [3, 4], installed in the cryostat in front of the EM calorimeter, provides a measurement of the energy lost upstream.

The EM calorimeter plays a crucial role during the operation of the LHC, since physics channels involving electrons and photons in the final state form a crucial part of the ATLAS physics program. Achieving the required precision and discovery reach places stringent requirements on the performance of the calorimeter. The uniformity of the calorimeter response over a large acceptance is particularly important for the overall resolution. This drives several design choices for the calorimeter: lead-liquid argon calorimetry provides a good energy resolution and homogeneity even in the presence of strong radiation; the accordion geometry (see Fig. 1) avoids readout cracks between calorimeter modules, thus also providing good uniformity.

In order to equalize the gains of different calorimeter channels, a calibration procedure involving electronic charge injection is used. This is however not sensitive to intrinsic characteristics of the ionization gaps in the liquid argon system, such as variations in gap sizes and LAr temperature changes. Such non-uniformities can be measured from the ionization signals created by charged particles. The calorimeter energy response to this ionization is not the best quantity for this purpose, because it requires a knowledge

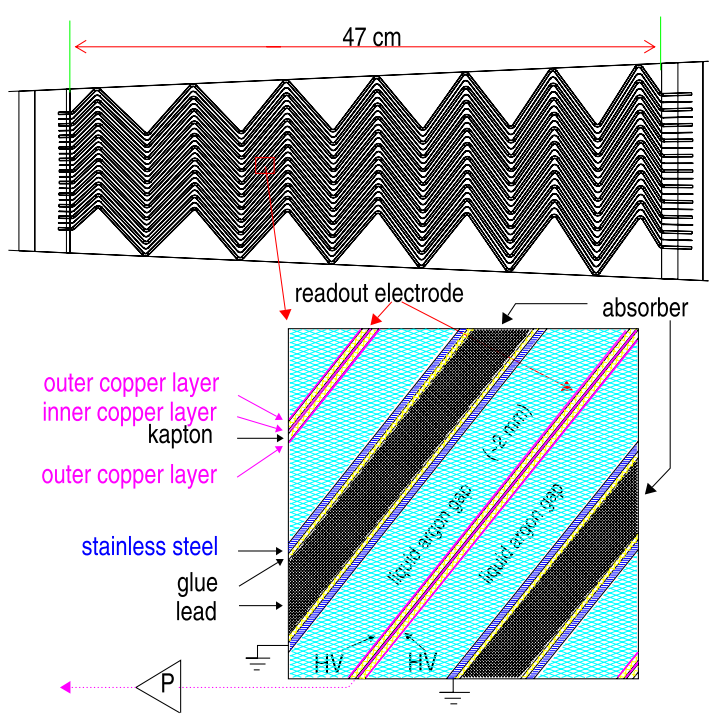

Fig. 1 Accordion structure of the barrel. The top figure is a view of a small sector of the barrel calorimeter in a plane transverse to the LHC beams. Honeycomb spacers, in the liquid argon gap, position the electrodes between the lead absorber plates of the energy of the incoming particle. However the electron drift time in LAr, which can be obtained from the signal pulse shape resulting from ionizing particles that deposit sufficient energy above the intrinsic noise level in a calorimeter cell, is a powerful monitoring tool. As explained in Sect. 2, the drift time is also about four times more sensitive to changes in the LAr gap size than is the energy response. Cosmic muons have been used to this end as part of the calorimeter commissioning before the LHC start-up.

The EM calorimeter installation in the ATLAS cavern was completed at the end of 2006. Before LHC start-up, the main challenge was to commission the associated electronics and automate all of the calibration steps for the full 173,312 channels. Cosmic muon data have been taken regularly for commissioning purposes since 2006. At the end of the summer and during autumn of 2008 stable cosmic muon runs were taken with the detector fully operational and using various trigger menus. In normal data taking only 5 samples around the pulse peak at $25 \mathrm{~ns}$ intervals are taken, but in order to accurately measure the drift time 32 samples are needed. The pulse height is also relevant, since larger pulses are less affected by electronic noise. A summary of the detector performance obtained from calibration data, cosmic muons and beam splash events is detailed in [5].

Measurements of the drift time $\left(T_{\text {drift }}\right)$ in the ATLAS EM calorimeter using cosmic muon data are presented in this paper. These drift times, which are independent of the amplitude of the pulses used for their determination, can be compared from one calorimeter region to another, and thus allow a measurement of the uniformity of the calorimeter.

\section{Ionization signal in the calorimeter}

The current resulting from the passage of a charged particle through a liquid argon gap has the typical ionizationchamber triangular shape, with a short rise time (smaller than $1 \mathrm{~ns}$ ) which is neglected in the rest of this note, followed by a linear decay for the duration of the maximum drift time

$T_{\mathrm{drift}}=w_{\mathrm{gap}} / V_{\mathrm{drift}}$,

where $w_{\text {gap }}$ is the LAr gap width and $V_{\text {drift }}$ the electron drift velocity [6]. The ionization current, $I$, is then modeled as:

$I\left(t ; I_{0}, T_{\text {drift }}\right)=I_{0}\left(1-\frac{t}{T_{\text {drift }}}\right) \quad$ for $0<t<T_{\text {drift }}$

where $I_{0}$ is the current at $t=0$. The peak current amplitude $I_{0}=\rho \cdot V_{\text {drift }}$ is proportional to the drift velocity and to the negative linear charge density $\rho$ along the direction perpendicular to the readout electrode, which varies with the lead 


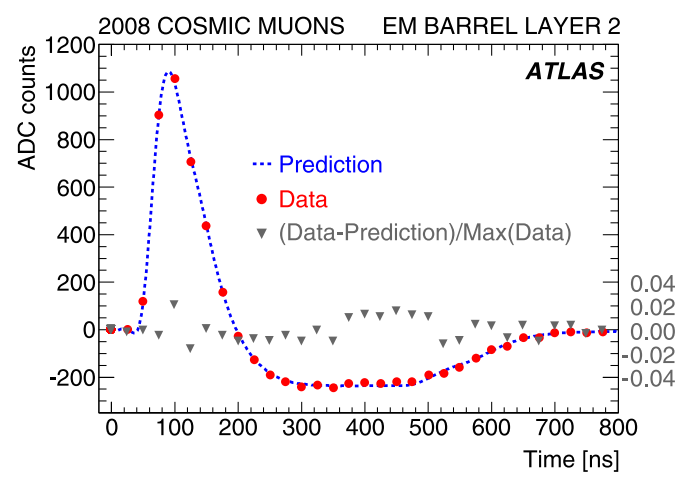

(a) Layer 2 of barrel

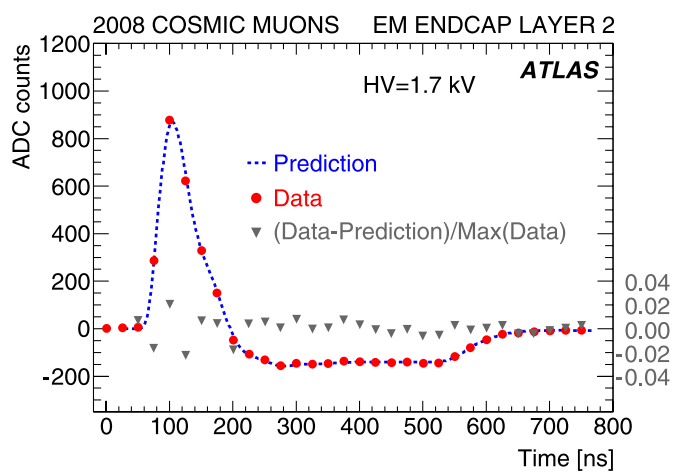

(b) Layer 2 of endcap

Fig. 2 Typical single ionization pulse in a cell of layer 2 of the barrel (a) and endcap (b) of the calorimeter. The large red dots show the data samples, the small blue dots the prediction and the grey triangles the

thickness. ${ }^{2}$ Since the determination of the energy is based on the measurement of $I_{0}$, it is crucial to be able to precisely evaluate and monitor $V_{\text {drift }}$. While the LAr gap thickness is mechanically constrained, the drift velocity depends on the actual conditions of the detector: the LAr temperature and density, and the local high voltage. Uniform response in a calorimeter with constant lead thickness requires uniform drift velocity in the gaps.

At this point it is appropriate to recall that each liquid argon electronic cell is built out of several gaps connected in parallel: for layers 2 and 3, there are 4(3) double-gaps in parallel in the barrel (endcap) respectively; there are four times as many gaps per cell in layer 1, given the coarser granularity of the readout in the azimuthal direction [1]. The parameters measured represent an average of the local gaps, both in depth along the cell, and in between the gaps forming a cell.

At the end of the readout chain the triangular signal is amplified, shaped and passed through a switched capacitor array which samples the signal every $25 \mathrm{~ns}$. The shaping function (see Sect. 3) includes one integration and two derivatives. Their net effect is to transform the triangular signal in a positive spike, followed by a flat undershoot, the length of the undershoot being equal to the drift time. The net area of the pulse, except for small fluctuations due to noise, being equal to 0 . Upon Level 1 trigger decision, the samples are then digitized using a fast-ADC and recorded [7, 8]. Figure 2 shows two typical digitized signal shapes, one for the barrel and the other for the endcap. The data samples in each plot correspond to a single cosmic muon event in a single cell, and fluctuations of the amplitude in each sample due to noise can be observed. The pulses shown pass the analysis

\footnotetext{
${ }^{2}$ If the LAr gap increases (as in the endcap) $\rho$ increases slightly on average due to showering in LAr. This is accounted for using detector simulation.
}

relative difference (data $(S)$ - prediction $(g)) / S_{\max }$, on the scale shown on the right side of the plot (normalized to the data)

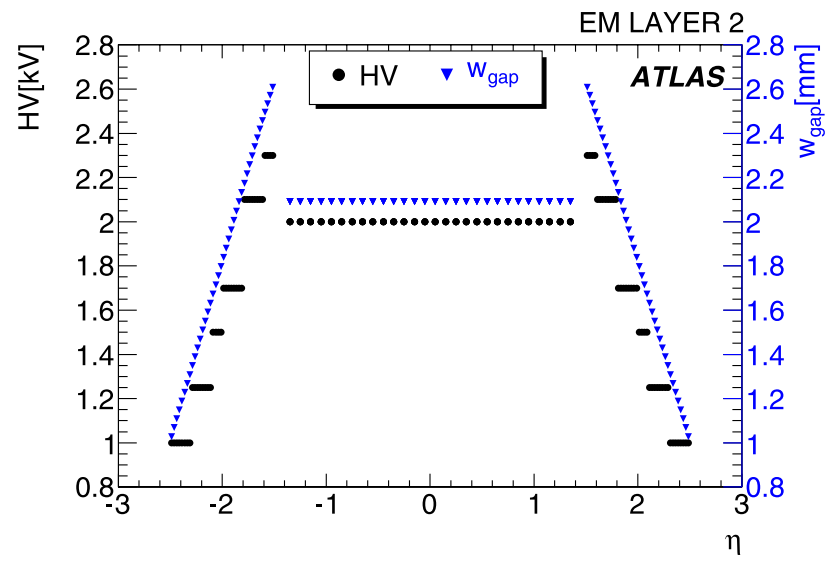

Fig. 3 Nominal HV (black dots) and nominal gap width $w_{\text {gap }}$ (blue triangles) versus $\eta$ in the 2nd layer of the EM calorimeter

criteria described in Sect. 4. The prediction is obtained by modeling the readout chain as described in Sect. 3. In the barrel section, the nominal gap size is constant $(2.09 \mathrm{~mm})$; in the endcap the gap size changes with pseudorapidity (see Fig. 3), so that at larger values of $\eta$ smaller gaps lead to a shorter pulse undershoot.

In the ideal case, an electrode is surrounded by two identical gaps, one on each side (see Fig. 4). Any modification of one of the gaps by a relative fraction $x$ will break the symmetry, leading to two different values of drift time $T_{D i}$ $(i=1,2)((4)$ and $(5))$. Figure 5 demonstrates this effect by showing the total collected current versus time in the case where the electrode is at the nominal position $\left(\delta_{\text {gap }}=0 \mu \mathrm{m}\right)$ or shifted by $100 \mu \mathrm{m}$ and $200 \mu \mathrm{m}$. This affects the rise at the end of the pulse (between 450 and $650 \mathrm{~ns}$ on Fig. 2(a) for example) which is sensitive to changes in the gap size over the charge collection area. The variation of the drift time inside the cell arises in part from the slight opening of the gaps along the accordion folds (see Fig. 1), but the bulk of the ef- 


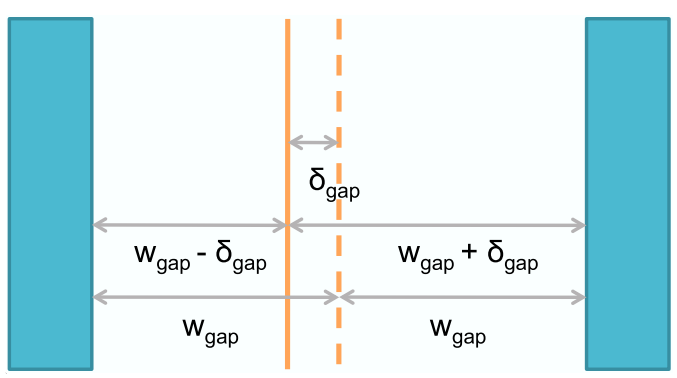

Fig. 4 Schematic view of a LAr gap. The nominal position of the readout electrode (dashed line) is exactly equidistant from the lead absorbers. Any shift with respect to the nominal position (solid line) causes an increase of the gap width on one side of the electrode, and a decrease on the other side

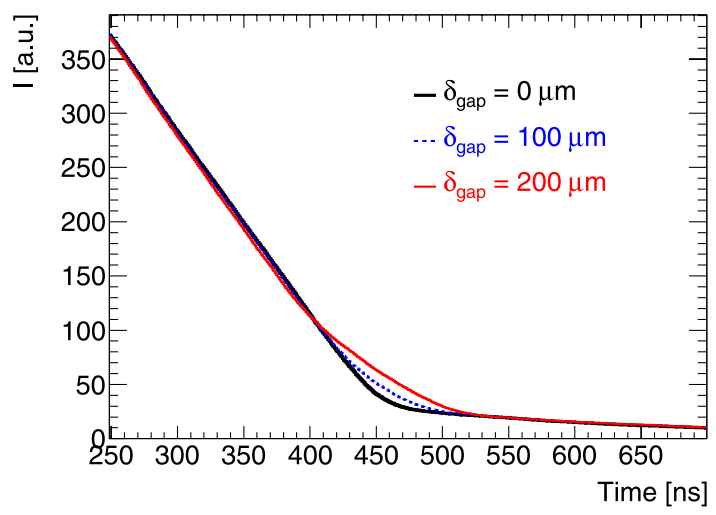

Fig. 5 Current as a function of time for a perfect centering of the electrode $\left(\delta_{\text {gap }}=0 \mu \mathrm{m}\right)$, a shift of $\delta_{\text {gap }}=100 \mu \mathrm{m}$ and $\delta_{\text {gap }}=200 \mu \mathrm{m}$

fect is caused by random or systematic displacements of the electrodes away from the gap center. Both effects are parametrized by the shift parameter $\delta_{\text {gap }}=x \cdot w_{\text {gap }}$. This shift parameter is limited to a maximum of $400 \mu \mathrm{m}$ due to the honeycomb filling the gaps, however, some modifications of electrical field lines (like edge effects) can contribute to local enlargements.

Beside the gap width, $w_{\text {gap }}$, the model of the signal takes into account the electrode shift parameter as well as possible variations in high voltage on both sides (neglecting in a first description the bend parts). The total signal can be expressed as a sum of two triangular signals, one for each side of the gap, each described by a drift time $T_{D i}$ and peak current $f_{i} \cdot I_{0}(i=1,2)$. Since the drift velocity $V_{\text {drift }}$ in liquid argon follows, for the range of electric fields relevant for this study, a power-law dependence on the electric field value $[9,10]$, with an exponent denoted here by $\alpha$

$V_{\mathrm{drift}}=K \cdot\left[\frac{H V}{w_{\mathrm{gap}}}\right]^{\alpha}$ the drift time and peak current fraction are given by:

$T_{D 1}=T_{\text {drift }}(1-x)^{1+\alpha}\left(H V_{1} / H V_{\text {nom }}\right)^{-\alpha}$,

$f_{1}=\frac{f_{\text {nom }}}{2}(1-x)^{-\alpha}\left(H V_{1} / H V_{\text {nom }}\right)^{\alpha}$,

$T_{D 2}=T_{\text {drift }}(1+x)^{1+\alpha}\left(H V_{2} / H V_{\text {nom }}\right)^{-\alpha}$,

$f_{2}=\frac{f_{\text {nom }}}{2}(1+x)^{-\alpha}\left(H V_{2} / H V_{\text {nom }}\right)^{\alpha}$,

where $T_{\text {drift }}$ and $f_{\text {nom }}\left(f_{\text {nom }}=1\right.$ when the bend parts are neglected) are respectively the drift time value and the fraction of current corresponding to the nominal high voltage $H V_{\text {nom }}$, and $H V_{i}$ corresponds to the actual high voltage applied on side $i$. In the barrel the nominal high voltage is $2 \mathrm{kV}$; in the endcap, the high voltage varies with $\eta$ (see Fig. 3) to cope with the varying gap, ensuring in principle a constant drift velocity by keeping the electric field constant. For the high voltage distribution, electrodes are grouped by sectors of $\Delta \eta \times \Delta \phi=0.2 \times 0.2$ and for redundancy separated supplies are used for each side of the electrodes. While in the vast majority of the sectors the high voltage has the nominal value, a few of them are operated at lower values, to prevent accidental sparking or excess noise.

Both in the barrel and in the endcap, the nominal operating field is close to $1 \mathrm{kV} / \mathrm{mm}$. The range of variation of $x$ (up to typically 20\%) induces a corresponding variation of the operating field of $\pm 20 \%$. In this reduced range, and for a fixed value of the liquid argon temperature, $88.5 \mathrm{~K}$, the variation of the drift velocity with the field is well described [9, 10] by a power law (3). Fitting the data published in [11] with such law gives $\alpha_{1}=0.316 \pm 0.030$. Additional information was obtained with our own data comparing a group of sectors in the barrel operated at $1600 \mathrm{~V}$, to the bulk operated at $2000 \mathrm{~V}$. The ratio of the velocity values obtained, taking into account small position dependence (see Sect. 6), gives: $\alpha_{2}=0.295 \pm 0.020$. Considering these two values, and given the low sensitivity of our results to the exact value of $\alpha$ (see Sect. 9) we decided to use $\alpha=0.3$ with a systematic uncertainty of ${ }_{-0.02}^{+0.04}$.

In the accordion geometry, the electric field in the bent sections has a lower value than in the flat parts. This leads to another contribution to the ionization signal in the form of two smaller triangular signals with a longer time constant and smaller $f_{\text {bend. }}$. The sum of the current fractions $\left(f_{\text {nom }}+f_{\text {bend }}\right)$ must be equal to 1 ; the main contribution on Fig. 5 is related to the drift time in flat sections, the tail at large time $(t>500 \mathrm{~ns})$ is due to the larger gap width in the bent sections of the accordion. These triangular shapes are parametrized (neglecting the electrode shift effect) by

$$
\begin{aligned}
& T_{D 3}=T_{\text {bend }}\left(H V_{1} / H V_{\text {nom }}\right)^{-\alpha}, \\
& f_{3}=\frac{f_{\text {bend }}}{2}\left(H V_{1} / H V_{\text {nom }}\right)^{\alpha},
\end{aligned}
$$


$T_{D 4}=T_{\text {bend }}\left(H V_{2} / H V_{\text {nom }}\right)^{-\alpha}$,

$f_{4}=\frac{f_{\text {bend }}}{2}\left(H V_{2} / H V_{\text {nom }}\right)^{\alpha}$.

In the barrel, the $T_{\text {bend }}$ and $f_{\text {bend }}$ contributions per layer are estimated using the GEANT4 simulation of a uniform charge density in the gap. These values are given in Table 1 for layers 1 to 3 (there are no bent sections in the presampler).

In the endcaps, for practical reasons a different simulation was used, MC GAMMA, where $10 \mathrm{GeV}$ electromagnetic showers have been simulated to predict the drift time $T_{\text {drift }}$ and to estimate $T_{\text {bend }}$ and $f_{\text {bend }}$. A photon simulation was chosen since the signals relevant to this study originate from electromagnetic showers produced by cosmic muons. The simulated photons were generated with a flight direction originating from the ATLAS Interaction Point. This differs from cosmic muons which cross the calorimeter in a quasivertical direction. Both $T_{\text {drift }}$ and $T_{\text {bend }}$ are plotted in Fig. 6 as a function of pseudorapidity for the three layers. These quantities are obtained from the distribution of the local drift time where the contributions from straight and bent sections of the accordion are clearly distinguished. Figure 6 shows that both quantities decrease with increasing $\eta$, following the reduction of the gap size. The difference observed between the layers is due to the depth variation of the gap size: the gap grows continuously from layer 1 to 3 due to the pro-

Table $1 T_{\text {bend }}$ and $f_{\text {bend }}$ values for the different layers in the barrel

\begin{tabular}{lll}
\hline Layer & $T_{\text {bend }}(\mathrm{ns})$ & $f_{\text {bend }}(\%)$ \\
\hline Layer 1 & 820 & 4.9 \\
Layer 2 & 898 & 7.1 \\
Layer 3 & 941 & 8.5 \\
\hline
\end{tabular}

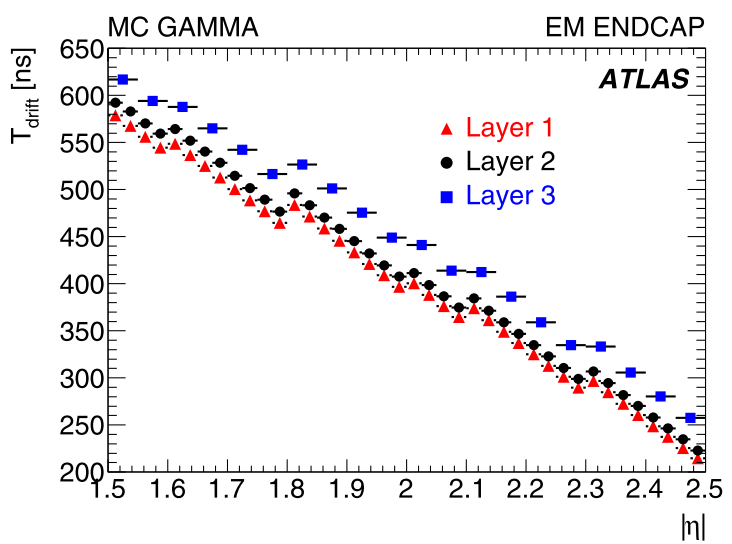

(a) MC $T_{\text {drift }}$ for endcap jective geometry of the cells. The values for layer 2 lie closer to those of layer 1 . This is explained by the fact that at the energy of the simulated showers $(10 \mathrm{GeV})$, the shower maximum is closer to layer 1 than to layer 3 . The current fraction $f_{\text {bend }}$ is also estimated from the simulation for every $\eta$ cell, with values ranging from $5 \%$ to $20 \%$ depending on pseudorapidity.

\section{Prediction of the ionization pulse shape}

The LAr calorimeters are equipped with a calibration system to inject an exponential pulse of precisely known amplitude onto intermediate "mother" boards located on the front face (for layer 1) and back face (for layers 2 and 3 ) of the calorimeter. The exponential decay time of these calibration signals has been trimmed to mimic the triangular ionization pulse shape as closely as possible. Since the readout path of the calibration signals is identical to that of the ionization pulses, the gain and pulse response of the electronics can be measured with the calibration system over the full range of signal amplitudes and time delays. The exponential calibration pulse properties are analytically modeled via two parameters $\tau_{\text {cali }}$ (inverse of the exponential slope) and $f_{\text {step }}$ (relative amplitude of a voltage step coming together with the main exponential signal).

The main ingredient needed for accurate energy and time reconstruction in the LAr EM calorimeter is the precise knowledge of the ionization signal shape in each readout cell, from which the optimal filtering coefficients [12] are computed. This knowledge of the ionization pulse shape is also necessary to accurately equalize the response across cells to account for its difference in shape and amplitude with respect to the calibration pulse. The difference between the two pulses is due to the slightly different shape of the

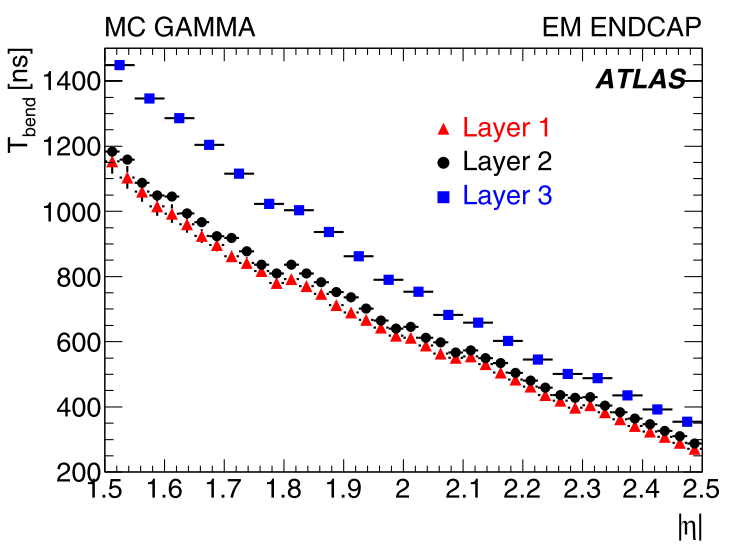

(b) MC $T_{\text {bend }}$ for endcap

Fig. 6 Monte Carlo simulation for (a) $T_{\text {drift }}$ and (b) $T_{\text {bend }}$ versus $\eta$ for the three endcap layers: layer 1 (red triangles), layer 2 (black dots) and layer 3 (blue squares) 
induced current (triangle versus exponential) and the different injection point for the currents (electrode versus mother board).

The prediction of the ionization pulse shape relies on the modeling of each readout cell as a resonant $R L C$ circuit (where $C$ corresponds to the cell capacitance, $L$ to the inductive path of the ionization signal and $R$ to the contact resistance between the detector cell and the readout line) and on the description of the signal propagation including reflections, amplification and shaping by the readout electronics.

In the standard ATLAS pulse shape prediction method, Response Transformation Method (RTM) [13], calibration pulses are used to determine the description of the signal propagation and the response of the readout electronics, as well as the parameters describing the electrical properties of the readout cell, ( $L C$ and $R C$ ) and the calibration signal ( $\tau_{\text {cali }}$ and $\left.f_{\text {step }}\right)$.

A second method has been developed for the EM barrel, First Principles Method (FPM) [14], where the signal propagation and the response of the readout electronics are analytically described, and the goodness of the analytical description is tuned using the measured calibration pulses.

Both methods need, as an input parameter, the value of the drift time in each cell, which can be either inferred from the local geometry of the detector along with the actual LAr temperature and high voltage, or measured from data pulses as described in this work. Details on the two methods, which describe the ionization pulse equally well, are given below.

\subsection{RTM method}

The properties of the signal propagation and of the electronic response of the readout of the LAr EM calorimeter cells are probed by the calibration system and can be determined from the measured calibration pulses. The two underlying assumptions behind the RTM [13] are that:

- The ionization pulse ( $\left.g_{\text {phys }}\right)$ can be numerically predicted from the corresponding calibration pulse $\left(g_{\text {cali }}\right)$ by means of time domain convolution with two simple functions, parameterizing respectively the shape difference between the exponential and triangular currents, and their different injection points in the detector, see [13]:

$$
\begin{aligned}
& g_{\text {phys }}(t) \\
& =g_{\text {cali }}(t) \\
& \quad * \mathcal{L}^{-1}\left\{\frac{\left(1+s \tau_{\text {cali }}\right)\left(s T_{\text {drift }}-1+e^{-s T_{\text {drift }}}\right)}{s T_{\text {drift }}\left(f_{\text {step }}+s \tau_{\text {cali }}\right)}\right\} \\
& \quad * \mathcal{L}^{-1}\left\{\frac{1}{1+s^{2} L C+s R C}\right\}
\end{aligned}
$$

where $\mathcal{L}^{-1}$ denotes an inverse Laplace transform, with $s$ being the variable in the frequency space. The first time-domain convolution corrects for the different signal shapes through the calibration pulse parameters $\tau_{\text {cali }}$ and $f_{\text {step }}$ and the drift time $T_{\text {drift }}$, while the second convolution accounts for the different injection points on the detector cell, modeled as a lumped $R L C$ electrical circuit.

- All parameters $\left(\tau_{\text {cali }}, f_{\text {step }}, L C, R C\right)$ used in the convolution functions, apart from the drift time, are directly extracted from the measured calibration pulses by numerical analysis [13].

\subsection{FPM method}

In the FPM method, the signal generation is based on "first principles" of signal propagation [14]. All the calculations are made in the frequency domain, and when the signal at the output of the final shaping amplifier is obtained, it is transformed to the time domain by using a fast Fourier transform [15].

After generation at the detector level, a signal is propagated along the signal cable, taking into account its impedance, propagation velocity, and absorption by the skin effect [14]. A small fraction of this signal is reflected at the signal cable-feedthrough transition, while the rest is transmitted. A second reflection takes place at the feedthroughpreamplifier transition. In this model, the feedthrough is modeled as a single cable section, with its own impedance, skin effect absorption constant, propagation velocity and length. The preamplifier is described by a complex impedance, the real part and the imaginary part $\left(\operatorname{Re}\left[Z_{\mathrm{PA}}\right]\right.$, $\left.\operatorname{Im}\left[Z_{\mathrm{PA}}\right]\right)$ being both functions of the frequency $\omega$. The last element of the chain is the $C R-R C^{2}$ shaping amplifier, described by the transfer function:

$F_{\mathrm{sh}}(\omega)=\omega \cdot \tau_{\mathrm{sh}} /\left(1+\left(\omega \cdot \tau_{\mathrm{sh}}\right)^{2}\right)^{3 / 2}$

where $\tau_{\mathrm{sh}}$ is the $R C$ time constant of this element. The model accounts for both the directly transmitted signal and the reflections up to the second order (i.e. two forwardbackward reflections and two backward-forward reflections).

Parameters are taken from construction (cable lengths, $f_{\text {step }}$ and $\tau_{\text {cali }}$, which were measured for all calibration boards [16]), from direct measurements channel-by-channel (resonance frequency $\omega_{0}=1 / \sqrt{L C}$ and $R$ ) [17], and from measurements on representative samples $\left(\operatorname{Re}\left[Z_{\mathrm{PA}}\right]\right.$, $\operatorname{Im}\left[Z_{\mathrm{PA}}\right]$, propagation velocity and skin effect constants). The signal cable impedance $Z_{\mathrm{S}}$ and the shaper time constant $\tau_{\text {sh }}$ were left as free parameters and fitted channel-bychannel on calibration pulses [14]. The values obtained for $Z_{\mathrm{S}}$ and $\tau_{\mathrm{sh}}$ came out close to expectations, giving confidence in the method which describes calibration pulses to $1 \%$ or better. The relative timing of all calibration signals was also reproduced with an accuracy of about $1 \mathrm{~ns}$. 
Table 2 Cut values for the most energetic sample of the data pulse. The approximate electronic cell noise $\left(\sigma_{\text {noise }}\right)$ averaged over layer and the approximate multiplicative conversion factor from ADC counts to $\mathrm{MeV}(F)$ are given as well

\begin{tabular}{lllll}
\hline & Layer & $S_{\max }(\mathrm{ADC}$ count) lower limit & $\sigma_{\text {noise }}(\mathrm{ADC}$ count $)$ & $F(\mathrm{MeV} / \mathrm{ADC}$ count $)$ \\
\hline \multirow{2}{*}{ Barrel } & Presampler & 200 & 8.0 & 7.0 \\
& Layer 1 & 500 & 8.0 & 2.5 \\
& Layer 2 $(|\eta| \leq 0.8)$ & 160 & 5.0 & 10.0 \\
& Layer 2 $(|\eta|>0.8)$ & 100 & 3.5 & 17.0 \\
& Layer 3 & 160 & 5.0 & 7.0 \\
\multirow{5}{*}{ Endcap } & Layer 1 & 500 & 7.0 & 3.0 \\
& Layer 2 & 160 & 4.0 & 14.0 \\
& Layer 3 & 160 & 2.0 & 7.0 \\
\hline
\end{tabular}

This method was not extended to the EM endcap because not all the necessary parameters have been measured with the required precision due to a more complex geometry.

\section{Description of the data}

Cosmic muon runs from the data-taking period of SeptemberNovember 2008 are used in this analysis, corresponding to a period where the LAr data acquisition system transmitted and saved 32 samples of the readout signals. The events of interest are those where muons lose a substantial fraction of their energy by radiation (the energy lost by $d E / d x$ in layer 2 is in average about $300 \mathrm{MeV}$ [5]). These events were triggered using calorimeter trigger towers over the full calorimeter depth, of size $\Delta \eta \times \Delta \phi=0.1 \times 0.1$ for $|\eta|<2.5,0.2 \times 0.2$ for $2.5<|\eta|<3.2$, and up to $0.4 \times 0.4$ for $3.2<|\eta|<4$.9. Since the data were collected from cosmic muons instead of LHC collisions, trigger thresholds were adjusted accordingly. For technical reasons, only cells which were readout in high gain (LAr readout has three gains with ratio $\sim 100 / 10 / 1$ ) are selected for this analysis. This has a very small impact on the selected sample as the energy deposits are typically in the high gain range (energies below $20 \mathrm{GeV}$ ).

Despite the small rate of cosmic muons depositing significant electromagnetic energy, the number of events recorded during the run period ensured sufficient statistics for most of the calorimeter regions, with the exception of the high- $\eta$ region of the endcaps. The pseudorapidity range in this study is hence restricted to $|\eta|<2.5$.

To minimize distortion of the signal shape, the energy deposited in a cell is required to be well above its typical noise value. This is particularly important since the drift time is obtained on an event-by-event basis. The quantity $S_{\max }$ is defined as the amplitude of the most energetic sample of the data pulse. The minimal required values for $S_{\max }$ are given in Table 2 for the different layers of the calorimeter; these values correspond to about $1-2 \mathrm{GeV}$. The average noise is also quoted, representing between 1 and $4 \%$ of the minimal value for $S_{\max }$. Unless differently stated, all ADC values are pedestal subtracted. The difference of thresholds between the $|\eta|<0.8$ and $0.8<|\eta|<1.4$ regions in layer 2 of the barrel is required by a difference in gain. To correct for this effect, the normalized variable $S_{\max }^{\text {gain }}$ is used for the selection, defined as $S_{\max }^{\text {gain }}=1.6 \cdot S_{\max }$ for $0.8<|\eta|<1.4$, and $S_{\max }^{\text {gain }}=S_{\max }$ everywhere else. An upper limit of 3900 ADC counts for $S_{\max }^{\text {gain }}$ plus pedestal is also required to avoid saturation.

As a small fraction of the ionization pulses are distorted and their drift times cannot be determined accurately, a set of cuts has been defined to select good quality pulses:

- The data should have a negative undershoot in the pulse shape. This is ensured by requiring that at least 5 samples after the peak have a negative amplitude.

- In order to prevent pulses with too short an undershoot (as can be the case for signals resulting from crosstalk for instance), a condition requires that the pulse does not contain more than 12 samples around 0 ADC counts at the end of the pulse. This condition cannot be applied to the endcap where such shapes occur due to smaller drift-time values at high pseudorapidity.

For a small fraction (6\%) of the LAr EM calorimeter the high voltage cannot be safely set to the nominal value. The cells belonging to these regions are excluded in the follow-

Table 3 Approximate number of cosmic muon induced pulses in each layer after quality cuts

\begin{tabular}{lll}
\hline & Layer & \# pulses \\
\hline \multirow{2}{*}{ Barrel } & Presampler & $20 \mathrm{k}$ \\
& Layer 1 & $43 \mathrm{k}$ \\
& Layer 2 & $331 \mathrm{k}$ \\
& Layer 3 & $79 \mathrm{k}$ \\
Endcap & Layer 1 & $13 \mathrm{k}$ \\
& Layer 2 & $45 \mathrm{k}$ \\
& Layer 3 & $18 \mathrm{k}$ \\
\hline
\end{tabular}


ing. The numbers of pulses per layer after quality cuts are given in Table 3.

\section{Extraction of the drift time}

The 32 data samples $S_{i}$ of each calorimeter cell selected by the criteria given in Sect. 4 are fitted using the pulse predictions described in Sect. 3, scaled by an amplitude $A_{\max }$ and shifted in time by an offset $t_{0}$ :

$$
\begin{aligned}
& g_{\text {fit }}\left(t ; A_{\max }, t_{0}, T_{\text {drift }}, x\right) \\
& \quad=A_{\text {max }} \cdot g_{\text {phys }}\left(t ; f_{\text {nom }}, T_{\text {drift }}, x, f_{\text {bend }}, T_{\text {bend }}\right)
\end{aligned}
$$

for $t>t_{0}$.

Four parameters are left free in this procedure: the drift time ( $T_{\text {drift }}$ ), the associated shift of the electrode estimated as $\delta_{\text {gap }}=x \cdot w_{\text {gap }}$ which is in fact only sensitive to the absolute value of $x$ when the high voltage is the same on both sides of electrodes, the global normalization factor $A_{\max }$ and the timing adjustment $t_{0}$. The optimal set of these four parameters is estimated using the least squares method to minimize the quantity:

$$
Q_{0}^{2}=\frac{1}{n-N_{p}} \sum_{i=1}^{n} \frac{\left(S_{i}-g_{\mathrm{fit}}\left(t_{i} ; A_{\mathrm{max}}, t_{0}, T_{\mathrm{drift}}, x\right)\right)^{2}}{\sigma_{\text {noise }}^{2}}
$$

where $n$ is the total number of data samples used in the fit (usually $n=32$ ), $N_{p}$ the number of free parameters $\left(N_{p}=4\right)$, and $\sigma_{\text {noise }}$ is given in Table 2. This minimization is performed using the MINUIT package [18].

Figure 7(a) presents the variation of $Q_{0}^{2}$ with $S_{\max }^{\text {gain }}$ for layer 2 of the barrel. An increase of the $Q_{0}^{2}$ value is observed when $S_{\max }^{\text {gain }}$ is larger. The same behavior is observed in the other calorimeter layers, as expected. In order to be able to apply a global selection to the fit quality independently of the data amplitude, a "normalized" $Q_{0}^{2}$, called $Q^{2}$, has been used:

$Q^{2}=\frac{1}{n-N_{p}} \sum_{i=1}^{n} \frac{\left(S_{i}-g_{\mathrm{fit}}\left(t_{i} ; A_{\max }, t_{0}, T_{\mathrm{drift}}, x\right)\right)^{2}}{\sigma_{\text {noise }}^{2}+\left(k S_{\max }\right)^{2}}$

where $k$ is chosen such that $Q^{2}$ is independent of $S_{\max }$, as represented in Fig. 7(b). The denominator in (12) is the quadratic sum of the noise and of the relative inaccuracy of the predicted shape. It represents the numerator uncertainty. The values of $k$ are given in Table 4 for the different layers of barrel (two methods) and endcap.

For the measurement of the drift time, the last data samples corresponding to the end of the pulse are very important. It was noticed that for a small fraction of pulses $(\sim 0.6 \%$ for the layer 2) the fit converges successfully but the predicted pulse does not succeed in describing the rise at the

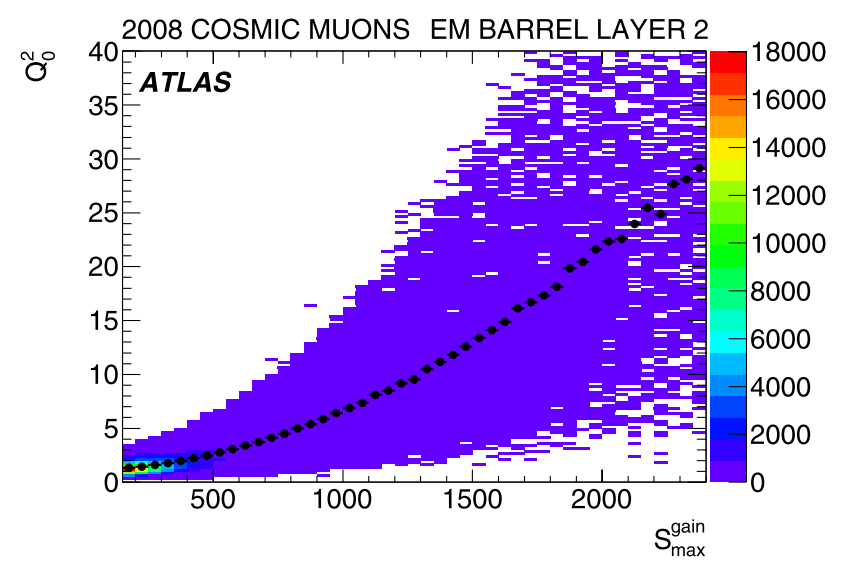

(a) $Q_{0}^{2}$ versus $S_{\max }^{\text {gain }}$

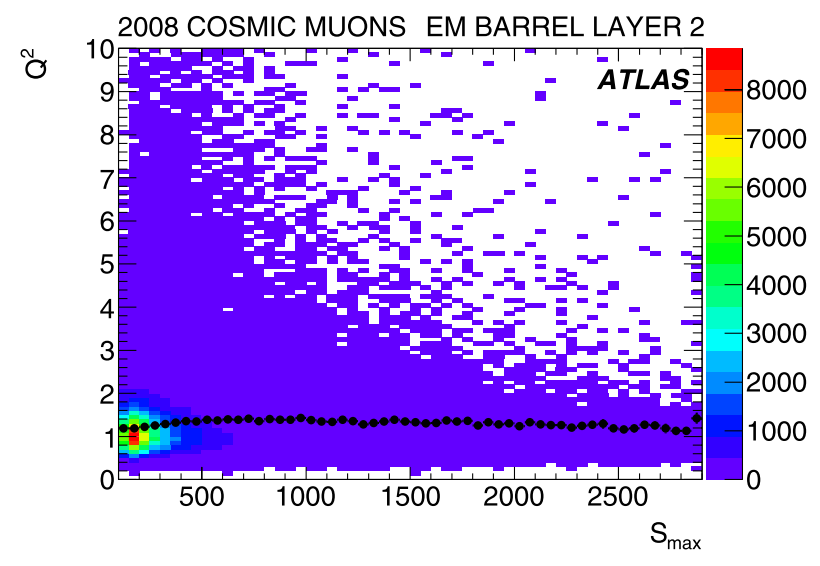

(b) $Q^{2}$ versus $S_{\max }$

Fig. 7 (a) $Q_{0}^{2}$ versus $S_{\max }^{\text {gain }}$ and (b) $Q^{2}$ versus $S_{\max }$ in layer 2 of the barrel. The black points correspond to the mean value

end of the pulse. This implies an incorrect estimate of the drift time. To specifically quantify the quality of the fit at the end of the pulse, the variable $\Delta_{\text {last7 }}$ has been defined, based only on the last 7 samples:

$\Delta_{\text {last7 }}=\sum_{i=26}^{32} \frac{S_{i}-g_{\text {fit }}\left(t_{i} ; A_{\max }, t_{0}, T_{\text {drift }}, x\right)}{S_{\max }}$.

Large values of $\left|\Delta_{\text {last }}\right|$ single out pulses with erroneous fitted drift times. This effect is also observed with a toy simulation, and therefore seems to be an intrinsic feature of the fitted function, with a large peak followed by a flat tail.

To remove events for which the end of the pulse is badly described by the model, a cleaning selection requiring $\left|\Delta_{\text {last }}\right|<0.15$ and $Q^{2}<2.5$ (3) in the barrel (endcap) is imposed.

An additional set of cuts on the maximum relative residual over all samples is applied for presampler cells, where pick-up of oscillatory signals was in a few places observed (3\% of the pulses): 
Table $4 k$ values for the different methods in the different regions of the EM calorimeter

\begin{tabular}{llll}
\hline Layer & $k_{\text {FPM }}$ in barrel & $k_{\text {RTM }}$ in barrel & $k_{\text {RTM }}$ in endcap \\
\hline Presampler & $0.9 \%$ & & \\
Layer 1 & $1.1 \%$ & $0.8 \%$ & $0.9 \%$ \\
Layer 2 & $0.8 \%$ & $1.0 \%$ & $1.4 \%$ \\
Layer 3 & $0.75 \%$ & $1.0 \%$ & $1.3 \%$ \\
\hline
\end{tabular}

- $\left|S_{i}-g_{\text {fit }}\left(t_{i}\right)\right|_{\max } / S_{\max }<10 \%$

- If the residual is small $\left(\left|S_{i}-g_{\text {fit }}\left(t_{i}\right)\right|_{\max }<20\right.$ ADC counts), the cut is relaxed to $\left|S_{i}-g_{\text {fit }}\left(t_{i}\right)\right|_{\max } / S_{\max }<20 \%$

After these selections, the fit parameters are examined in more detail. Figure 8 presents the distribution of the absolute value of the shift parameter, $\delta_{\text {gap }}=x w_{\text {gap }}$, as a function of the drift time.

The region in Fig. 8(a) with a drift time $T_{\text {drift }}$ comprised between 380 and 550 ns corresponds to the expected range for the drift time in the barrel given the resolution of the measurement. The low drift time region $T_{\text {drift }}<$ 380 ns of Fig. 8(a) $(0.05 \%$ of the pulses $)$ is dominated by low-amplitude pulses distributed evenly in the calorimeter. A closer examination shows that in about $80 \%$ of the cases for the layer 2 barrel, signals in excess of $S_{\max }=1500 \mathrm{ADC}$ counts or cells sampled at medium gain are found as first neighbors which corroborates a crosstalk hypothesis.

In the region $T_{\text {drift }}>550$ ns of Fig. 8(a) $(0.25 \%$ of the pulses), some pulses are still significantly negative, more than $700 \mathrm{~ns}$ after the time of signal maximum. A possible explanation is that the energy deposit originates from a photon emitted along a bent section, thus having an abnormally enhanced $f_{\text {bend }}$ contribution. Unfortunately the runs taken with 32 samples do not contain information from the inner tracker which would have allowed this hypothesis to be validated by a projectivity study. Aside from these extremely large drift time pulses, there is a larger class of pulses which are only somewhat longer than normal. They are distributed along specific $\eta$ and $\phi$ directions: in the transition regions at $|\eta|=0.8$ and between the two half-barrels at $\eta=0$ (see Sect. 6.1.1) where a slight dilution or leakage of the electric field lines yields a larger drift time (this is also observed in layer 1 of the barrel); in the intermodular regions in $\phi$ in the upper part of the detector, where mechanical assembly tolerances allow for a slightly increased gap at the interface between modules due to gravity effects (this is not seen in barrel layers 1 and 3 which are much closer to the mechanical fixed points).

In the endcap, the cloud of points corresponding to the expected $T_{\text {drift }}$ is broader than in the barrel, as can be seen in Fig. 8(b): it ranges from 300 to $600 \mathrm{~ns}$ as a consequence of the gap size variation with $\eta$ of the endcap design. The fact that the dispersion of $\left|\delta_{\text {gap }}\right|$ is larger at higher values of $T_{\text {drift }}$ is explained as a consequence of the larger gap size: the larger the gap width, the larger the displacement of the electrode can be. A few events $(0.9 \%$ of the pulses) are observed at very high values of both $T_{\text {drift }}$ and $\left|\delta_{\text {gap }}\right|$. They are located at low $|\eta|$ where the drift time is very large by construction (see Fig. 6(a)). Their pulse shape cannot be completely readout using 32 samples, and in particular the rise following the undershoot is partially absent, which leads to unphysical values of the shift above $400 \mu \mathrm{m}$.

A distinctive aspect of the fit, which is clearly visible in Fig. 9, is that it yields a peak at $\left|\delta_{\text {gap }}\right|=0$. This is mainly explained by noise fluctuations. The superposition of two triangles of ionization current with unequal length due to an electrode shift (see Fig. 5) can only lead to a softening

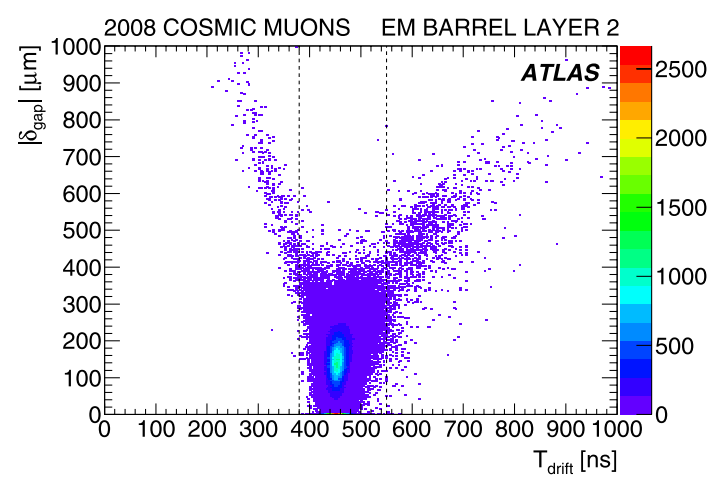

(a) Layer 2 of the barrel

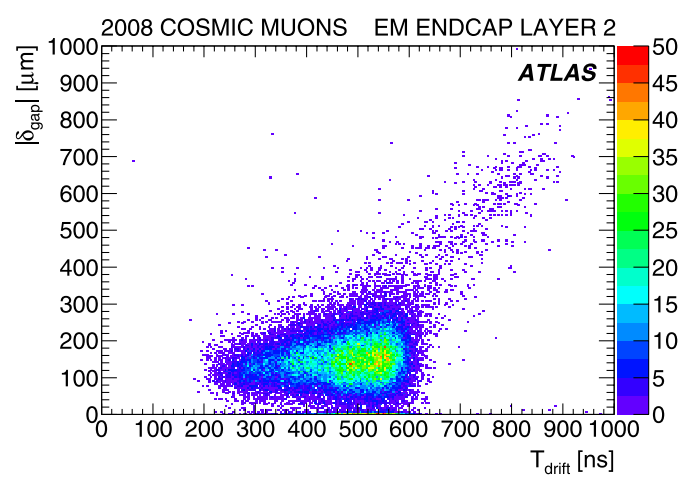

(b) Layer 2 of the endcap

Fig. 8 Absolute value of the shift parameter as a function of the drift time in the barrel (a) and in the endcap (b), for layer 2 


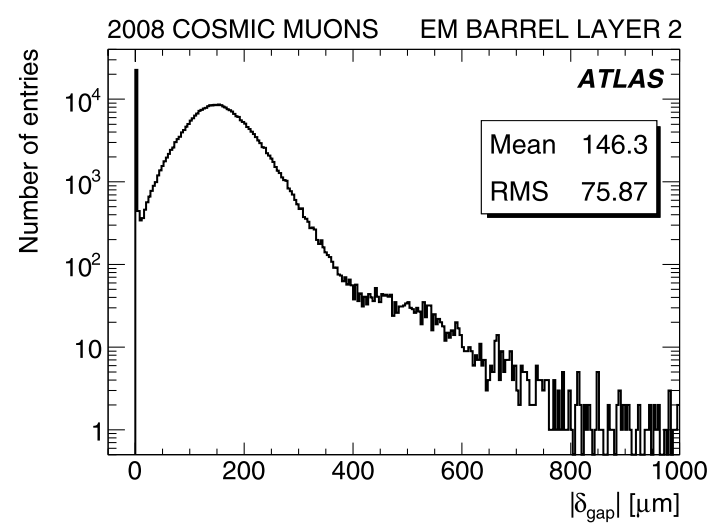

(a) Layer 2 of the barrel

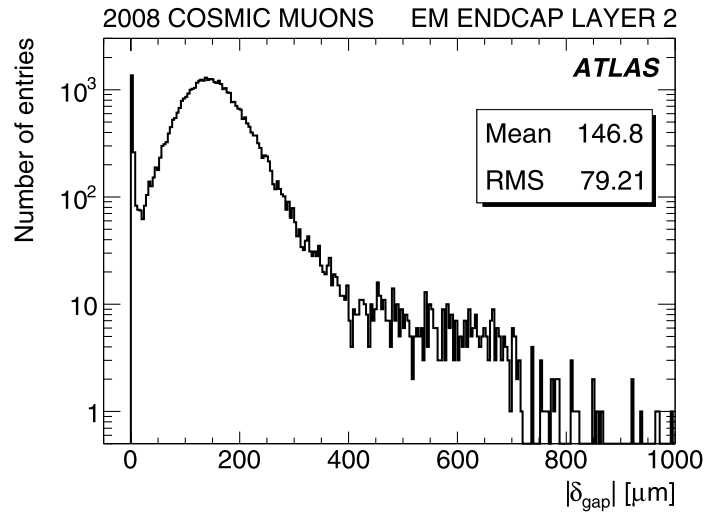

(b) Layer 2 of the endcap

Fig. 9 Distribution of the absolute value of the shift parameter in layer 2 of the barrel (a) and endcap (b)

of the rise at the end of the pulse, compared to the singletriangle case. If, due to noise, the rise is steeper than for a single-triangular shape, the fit forces $\delta_{\text {gap }}$ to 0 . In order to improve the statistical significance of high-amplitude signals and minimize the impact of noise fluctuations, it has been decided to weight the events by $\left(S_{\max }^{\text {gain }}\right)^{2}$. The results in the following sections of this note are produced with this weighting factor.

\section{Results in the calorimeter barrel}

Two parallel analyses have been performed for this part of the calorimeter using the two pulse shape prediction methods described in Sect. 3. The analyses agree at the level of $0.3 \%$, which provides a good check of the robustness of these results. In this section the measurement of the drift time is presented, along with its implications for the calorimeter response uniformity and an estimation of the electrode shift.

\subsection{Drift time measurement \\ in pseudorapidity and azimuthal angle}

Results in layer 2 are presented first because the statistical uncertainties are lower in this layer (see Table 3). More refined comparisons between the two methods are then possible. The following subsection reports on the results in the other layers. The presampler is discussed separately due to its different structure.

\subsubsection{Layer 2 of the barrel}

Figure 10 presents the drift time $T_{\text {drift }}$ extracted from the fit as a function of $\eta$. The results of the two methods differ by $0.1 \mathrm{~ns}$ on average with an RMS of $1.3 \mathrm{~ns}$. The full purple line illustrates the prediction from absorber thickness measurements made during the calorimeter construction [2]. This prediction is based on the fact that the mechanical structure of the calorimeter ensures that the pitch (with nominal values shown in parentheses) is constant to within about $5 \mu \mathrm{m}$ :

$$
\begin{aligned}
& \operatorname{Absorber}(2.2 \mathrm{~mm})+w_{\text {gap }}(2.09 \mathrm{~mm}) \\
& \quad+\operatorname{Electrode}(0.280 \mathrm{~mm})+w_{\text {gap }}(2.09 \mathrm{~mm}) \\
& =6.66 \mathrm{~mm}=(2 \pi / 1024) \cdot R_{i} \cos \theta_{i}
\end{aligned}
$$

where $R_{i}$ and $\theta_{i}$ are the average radius and the local angle of the 1024 accordion-shaped absorbers with respect to the radial direction. So if the thickness of the absorber varies with $\eta$, the gap will also vary in the opposite direction. As the drift time $T_{\text {drift }}$ is directly related to the gap by:

$T_{\text {drift }}=T_{D 0}\left(w_{\text {gap }} / w_{\text {gap } 0}\right)^{1+\alpha}$

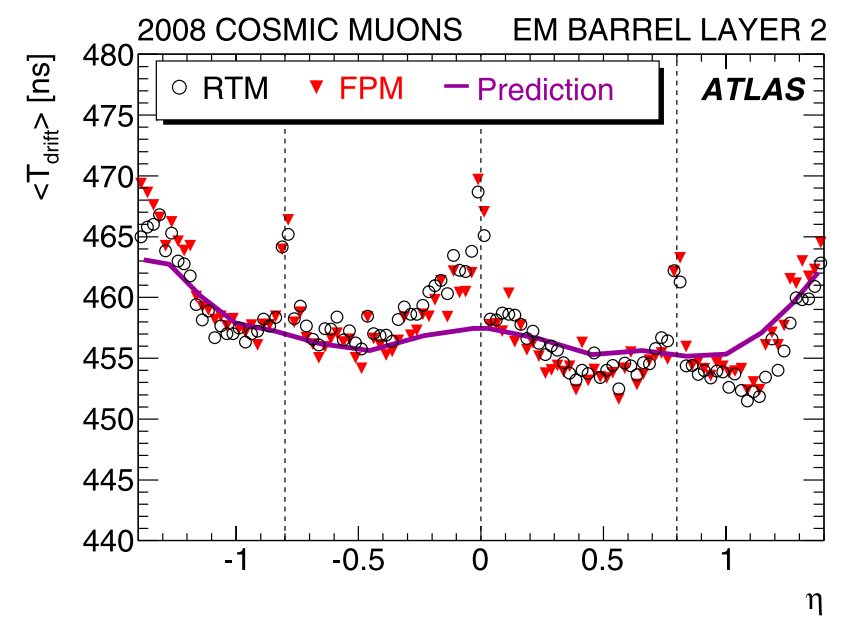

Fig. 10 Drift time as a function of $\eta$ in layer 2 of the barrel: using the RTM method (open dots), the FPM method (red triangles) and the prediction described in the text (purple line) 


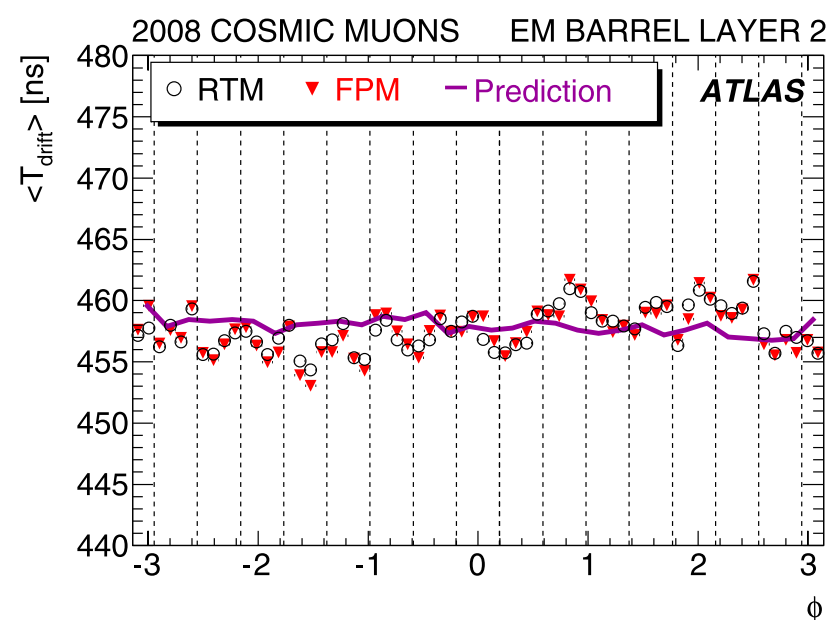

Fig. 11 Drift time as a function of $\phi$ in layer 2 of the barrel: using the RTM method (open dots), the FPM method (red triangles) and the prediction described in the text (purple line)

a prediction can be derived for the drift time from the variations around the nominal gap size $\left(w_{\text {gap } 0}=2.09 \mathrm{~mm}\right)$ associated with $T_{D 0}=\left\langle T_{\text {drift }}\right\rangle=457.9 \mathrm{~ns}$.

The agreement between the prediction coming from precision mechanical probe measurements of the absorber thickness and the data is rather good, except in the transition regions around $\eta=0, \pm 0.8$ and -1.4 , where the lower field induces a larger $T_{\text {drift. }}$ To quantify the agreement between the drift time measurements from the fit and the estimate from the measurement of the absorbers, the RMS of the difference between the data points and the prediction is computed. This yields a value of $2.9 \mathrm{~ns}$, as compared to an RMS deviation with respect to a constant value of $3.7 \mathrm{~ns}$, excluding the data points around the transition region in each case. Comparing bin by bin the drift times obtained (Fig. 10) for the negative and positive values of $\eta$, one gets a distribution with a mean of $3.4 \pm 0.2 \mathrm{~ns}$ and RMS of $1.7 \mathrm{~ns}$. The predicted value is $1.5 \pm 0.2 \mathrm{~ns}$.

The $T_{\text {drift }}$ distribution as a function of $\phi$ is presented in Fig. 11, for both methods. There is a small difference between the $\phi<0((456.8 \pm 0.3) \mathrm{ns})$ and $\phi>0((458.3 \pm$ $0.3)$ ns) regions: a $(0.3 \pm 0.1) \%$ relative effect consistent with sagging and pear shape deformation of the calorimeter. No significant variations are observed in the absorber thickness measurements. The distribution of the results is also rather uniform when looking at the two half-barrels separately. The RMS of the $\phi$ distribution is smaller (1.8 ns) when the two half-barrels are combined, than for the $\eta<0$ (2.8 ns) and $\eta>0$ (3.1 ns) half-barrels separately. This may be due to the existence of small $\phi$ modulations with opposite phases in the two half-barrels that appear to be more visible in layer 3 (see Fig. 12).

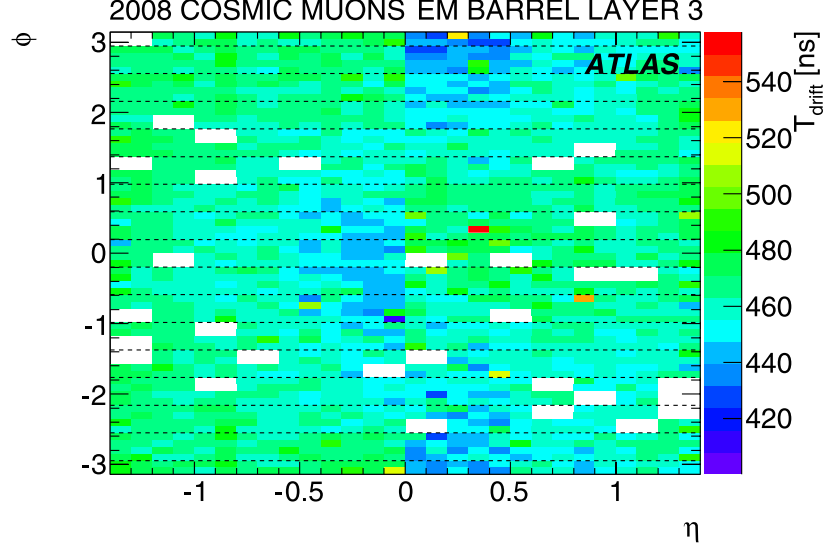

Fig. 12 2D map of $T_{\text {drift }}$ in $(\eta, \phi)$ for layer 3 . The empty bins correspond to sectors with non nominal HV

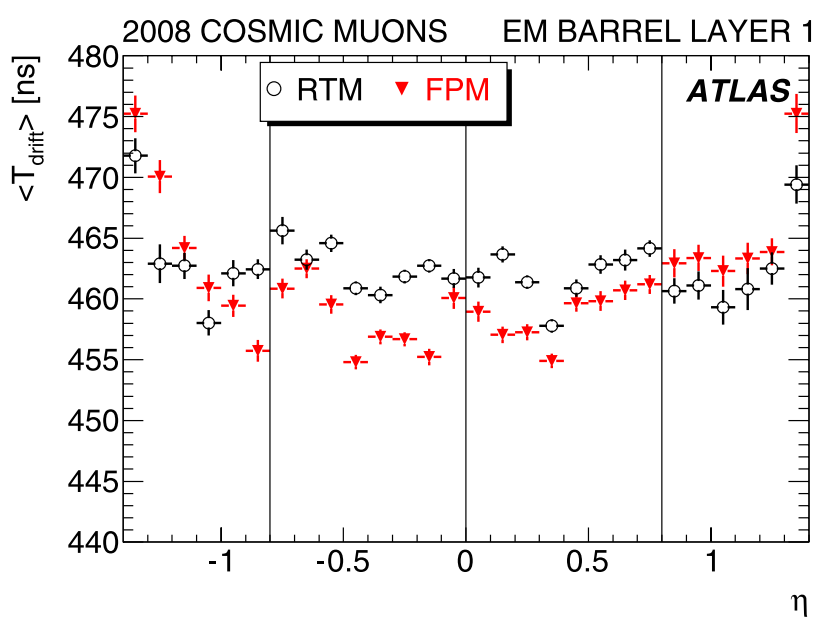

Fig. 13 Drift time as a function of $\eta$ in layer 1 of the barrel: using the RTM method (open dots) and the FPM method (red triangles)

\subsubsection{Other layers of the barrel}

The distribution of $T_{\text {drift }}$ as a function of $\eta$ for layer 1 is displayed in Fig. 13. The results of the two methods differ by $1.3 \mathrm{~ns}$ on average, with an RMS of $4 \mathrm{~ns}$, and at some points by up to $7 \mathrm{~ns}$. The front layer is particularly intricate because of the large relative variations of the cell depths which present a discontinuity at $|\eta|=0.8$, inducing a corresponding variation of the cell capacitance and bent-to-flat ratio. Given that the two methods differ in their estimation of the cell capacitance, such a difference is not unexpected.

In Fig. 12, a drift time modulation with $\phi$ is clearly visible for $|\eta|<0.5$ (and equally present in both methods) in both half barrels of layer 3 . While the source of the modulation is so far unexplained, the fact that the modulations in the two half-barrels are opposite in phase is expected, since one of the half-barrels was rotated by 180 degrees about the vertical direction for final integration. 


\subsubsection{Presampler}

The presampler is constructed differently from the other layers of the calorimeter. It is made of narrow flat electrodes. The size of the gaps is slightly smaller than elsewhere, leading to values of $T_{\text {drift }}$ lower than in the rest of the calorimeter. In addition, this gap varies with $\eta$; the values for the 4 regions are given in Table 5. The effect on the fitted drift time can be immediately seen in Fig. 14. The prediction superimposed on the measured distribution is normalized to the region $0.8<|\eta|<1.2$. Good agreement within $1 \%$ is observed between the measured and expected drift times as a function of $\eta$. As there are no bent sections in the presampler, the pulse description is simpler than in the case of the other layers. While the variations in $\eta$ are large, the $\phi$ dependence of the drift time is negligible.

\subsection{Response uniformity}

The reconstructed value of the energy deposited in the calorimeter by an electron or photon should be independent of the position of its impact on the calorimeter. The nonuniformity coming from local variations of the response due to gap fluctuations can be determined using the drift time

Table 5 Gap values in presampler

\begin{tabular}{ll}
\hline$\eta$ region & $w_{\text {gap }}$ (in $\left.\mathrm{mm}\right)$ \\
\hline$|\eta|<0.4$ & 1.966 \\
$0.4 \leq|\eta|<0.8$ & 1.936 \\
$0.8 \leq|\eta|<1.2$ & 2.006 \\
$1.2 \leq|\eta|$ & 1.906 \\
\hline
\end{tabular}

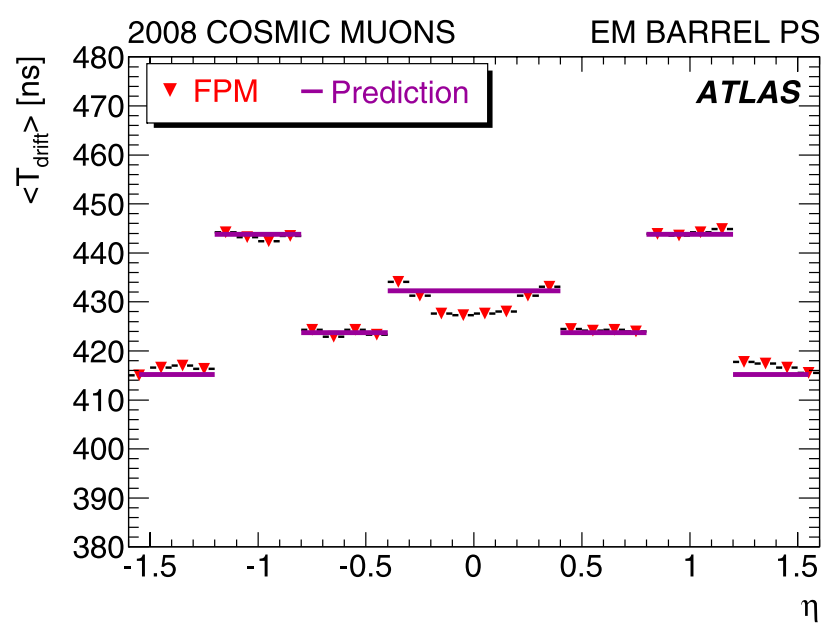

Fig. 14 Drift time as a function of $\eta$ in the presampler barrel using the FPM method (red triangles). The full purple line represents the prediction normalized to the region $0.8<|\eta|<1.2$, using (15) and the gap values given in Table 5. The empty bins correspond to sectors with non nominal HV

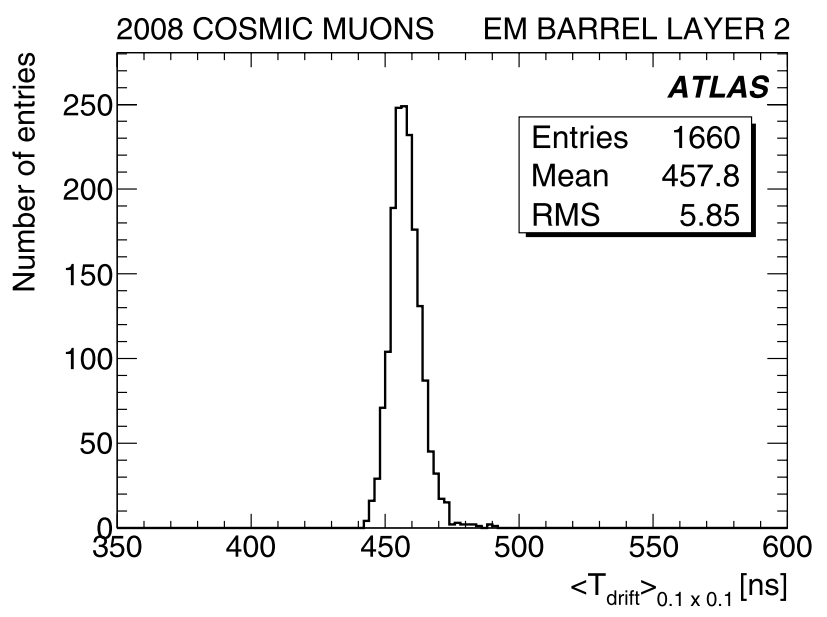

Fig. 15 Drift time uniformity between groups of $4 \times 4$ cells $(\Delta \eta \times \Delta \phi=0.1 \times 0.1)$ for barrel layer 2

measurements. This study is done only for layer 2 , which is the main contributor to the energy response of the detector as it collects $\sim 70 \%$ of the total electromagnetic signal in the calorimeter.

Figure 15 shows the distribution of the drift time averaged over groups of $4 \times 4$ cells corresponding to an area of $0.1 \times 0.1$ in $\Delta \eta \times \Delta \phi$ plane. This area represent a typical transverse size of a single particle shower. The average of the statistical uncertainties on $T_{\text {drift }}$ obtained for pulses within the various $4 \times 4$ groups is $1.25 \mathrm{~ns}$, well below the dispersion of the determined $T_{\text {drift }}$ values of the groups (the RMS is $5.85 \mathrm{~ns}$ ). From the measurement of drift times, the systematic dispersion of the gaps can be estimated and its impact on the calorimeter energy response can be assessed.

The drift time uniformity, corresponding to the ratio of the RMS and the mean value of the local $T_{\text {drift }}$ distribution amounts to $(5.85 \pm 0.14) / 457.8=(1.28 \pm 0.03) \%$. From the relation between the drift time and the drift velocity (1), the latter being proportional to the energy response, together with (15), it follows, that the drift time uniformity leads to a dispersion of the response due to the gap variations of $(1.28 \pm 0.03) \% \cdot(\alpha /(1+\alpha))=(0.29 \pm 0.01) \%$. Excluding transition regions in $\eta$ and in $\phi$, the gap variations amount to $5.7 / 457.4=1.25 \%$ and the impact on the response is $0.28 \%$. Taking into account small variations observed in the result when changing the weighting, the fit strategy (see Sect. 9) or the pulse reconstruction method, a systematic error of $0.03 \%$ is obtained. The uncertainty on $\alpha$ (see Sect. 2), treated here as external parameter, contributes with a systematic uncertainty of ${ }_{-0.02}^{+0.04}$. Grouping all errors together in quadrature gives as the final result: $\left(0.29_{-0.04}^{+0.05}\right) \%$.

\subsection{Electrode shift}

As presented in Sect. 2, there is some freedom for the electrodes to be displaced with respect to their nominal posi- 


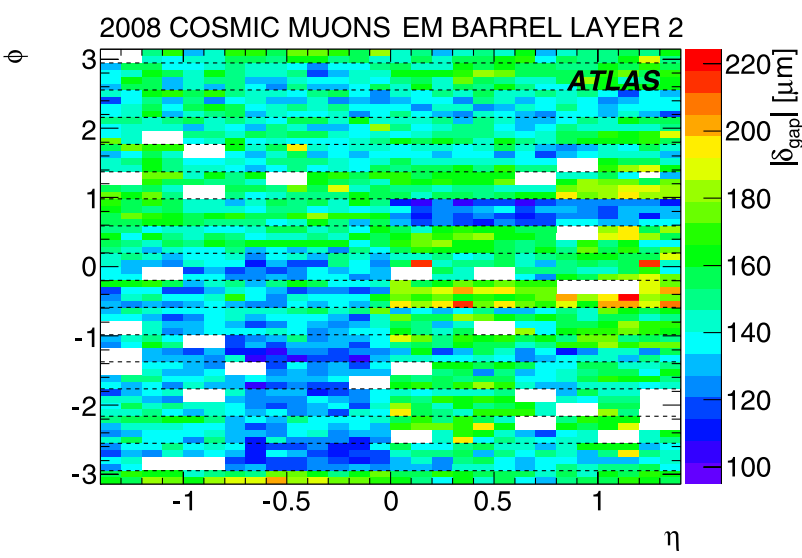

Fig. $16(\eta, \phi)$ map in which $\left|\delta_{\text {gap }}\right|$ is plotted per bin of $0.1 \times 0.1$

tions equidistant between two neighboring absorbers. This displacement is expected to be less than $400 \mu \mathrm{m}$ except perhaps in the transition regions between modules.

The electrode shift is left as a free parameter in the fit to the data, which yields one value per calorimeter cell. Only the average of the absolute value of the displacement can be observed. Since a cell consists of several electrodes, an effective value is obtained which is a combination of the individual movements of each electrode within a cell.

The local average value for the shift parameter per bin of $0.1 \times 0.1$ is shown in Fig. 16 for layer 2. It indicates that the bottom half (negative $\phi$ ) of the negative- $\eta$ half-barrel has shift parameter values somewhat lower than average. Similarly the module azimuthally located between $4 \pi / 16$ and $5 \pi / 16$ in the $\eta>0$ half-barrel presents lower shift values. These variations given their distribution throughout the detector, are likely to be due to mechanical construction issues.

The shift parameter also covers local variations of the "double-gap" within a cell, for example, by the slight opening of gaps along an accordion fold. This latter variation is in general much smaller than the off-centering of electrodes between absorbers.

Smaller values of the shift parameter are expected for the presampler compared to the accordion layers, due to mechanical constraints on the electrodes which are individually glued in between two precision structural frames [2]. The mean value of the shift in the presampler is found to be $\left\langle\left|\delta_{\text {gap }}\right|\right\rangle=66.5 \mu \mathrm{m}$, as compared to $146 \mu \mathrm{m}$ in the accordion section.

\section{Results in the calorimeter endcap}

As was done for the barrel, the endcap results are grouped in three different parts: drift time measurements, calorimeter response uniformity and electrode shift determination.

\subsection{Drift time measurement \\ in pseudorapidity and azimuthal angle}

The drift time $T_{\text {drift }}$ averaged over $\phi$ is studied as a function of $\eta$ for each of the three layers of the endcap (see Fig. 17). The two endcaps, $\mathrm{A}(\eta>0)$ and $\mathrm{C}(\eta<0)$, are combined in the figure. A general decrease of $T_{\text {drift }}$ with increasing pseudorapidity is observed, as expected from the corresponding reduction of the design gap size. Fewer fluctuations are observed in layer 2 , which offers a larger cross section to cosmic muon-induced electromagnetic showers. In all layers regular steps are observed, corresponding to the locations of the boundaries between high voltage regions.

The data are compared to the Monte Carlo calculation described in Section 2. Good agreement is observed at high $\eta$, however the Monte Carlo is slightly above the data at low values of $\eta(\sim 1-3 \%)$, which is a more difficult region to simulate.

In Fig. 18, for a comparison, the data points from the three distributions of Fig. 17 are super-imposed on the same plot. An increase of the drift time with the cell gap size at fixed $\eta$ is clearly observed, with $T_{\text {drift }}$ being smallest for layer 1 and highest for layer 3 (see Sect. 2 and Fig. 6(a)). The drift time for layer 2 lies half way between layers 1 and 3 in contrast to the Monte Carlo simulation (Fig. 6(a)) where the values for layer 2 are closer to the values of the layer 1 . This difference reflects the fact that cosmic muons are randomly distributed within the depth of layer 2 , while the photons of the simulation develop there shower closer to layer 1.

Figure 19 shows the drift time $T_{\text {drift }}$ as a function of azimuthal angle for layer 2 for the two endcaps. The values of $T_{\text {drift }}$ for each given pseudorapidity bin have been normalized to the average in order to mask the dependence on $\eta$. Vertical dashed lines indicate the boundaries between modules. An asymmetry is visible on Fig. 19 between positive and negative values of $\phi: T_{\text {drift }}(\phi>0)$ is larger $(0.996 \pm 0.002)$ than $T_{\text {drift }}(\phi<0)(0.980 \pm 0.002)$. Since $\phi<0$ is the lower half of the calorimeter, we associate this effect to the greater gravitational compression of this part leading to slightly smaller gaps than in the upper half $\phi>0$.

\subsection{Response uniformity}

An estimate of the intrinsic uniformity of the endcap can be made in a similar manner as presented for the barrel in Sect. 6.2. The average drift time across a region of size $0.1 \times$ 0.1 on the $(\eta, \phi)$ plane is computed, with special care to take into account the varying gap thickness.

Figure 20 represents the distribution of $T_{\text {drift }} /\left\langle T_{0}\right\rangle$ for layer 2. The normalization $\left\langle T_{0}\right\rangle$ corresponds to the value (per $\eta$ cell) predicted from a first order polynomial fit to the data 


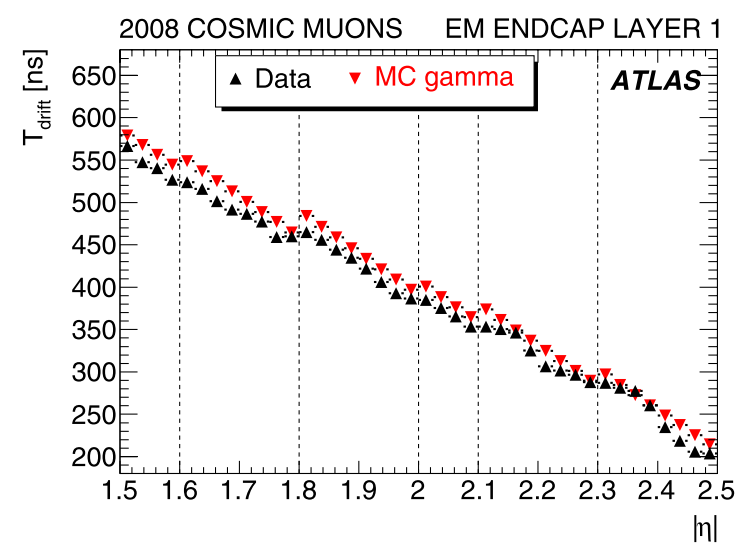

(a) Layer 1

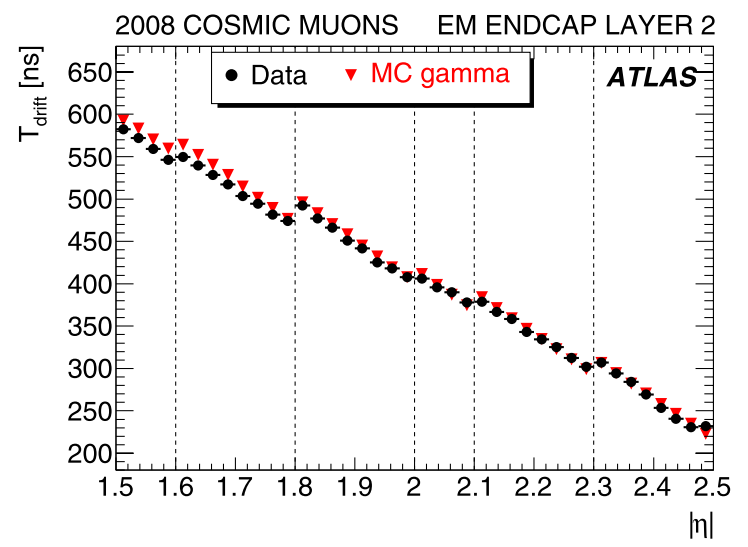

(b) Layer 2

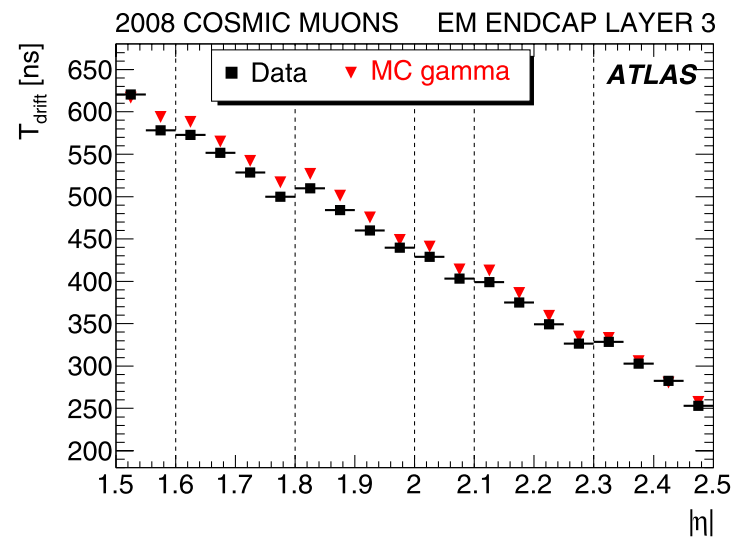

(c) Layer 3

Fig. 17 Drift time versus pseudorapidity for layer 1 (a), layer 2 (b), and layer 3 (c) cells of the endcap. Black points are the data and red triangles Monte Carlo predictions for photons. The vertical dashed lines show the boundaries between different high voltage regions

$T_{\text {drift }}$ in each high voltage region. This normalization cancels out the change of the drift time due to the nominal design gap size variation with $\eta$. The study is carried out only

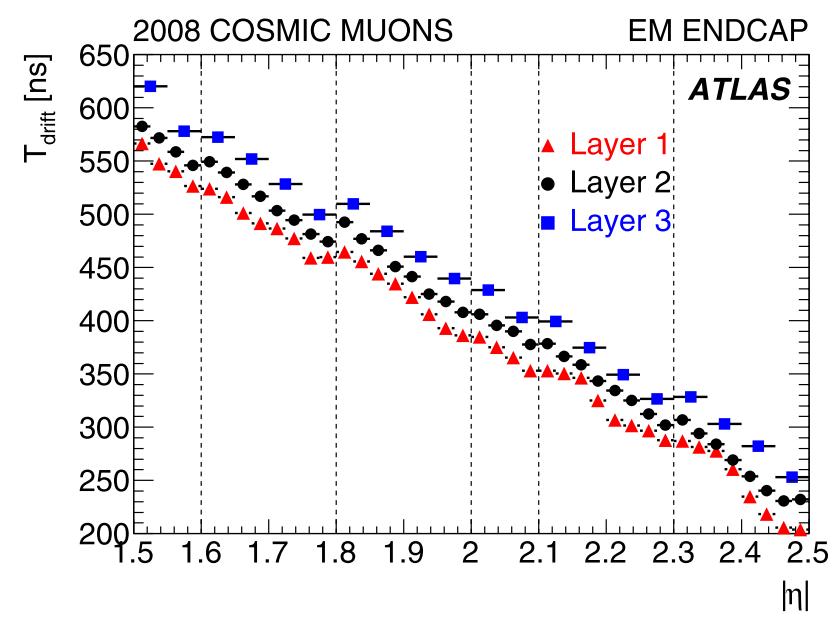

Fig. 18 Drift time versus pseudorapidity for the three layers of the endcap: layer 1 (red triangles), layer 2 (black dots), layer 3 (blue squares). The vertical dashed lines show the boundaries between different high voltage regions

for layer 2 since it contains most of the shower energy of typical LHC electrons and photons. In addition, more events have been recorded in layer 2 than in the other layers, which increases the statistical accuracy of the measurement.

The drift time uniformity of the $T_{\text {drift }}(0.1 \times 0.1)$ distribution has an RMS of $(2.8 \pm 0.1) \%$. To get the pure systematic non-uniformity between the $0.1 \times 0.1$ cells, the dispersion within the $0.1 \times 0.1$ cells, which in this case is not negligible, $(1.5 \pm 0.1) \%$, is quadratically subtracted. These numbers translate to a uniformity of the endcap calorimeter response due to intrinsic gap variations of $(0.54 \pm 0.02) \%$. Systematic effects as discussed in Sect. 9 and the uncertainty on $\alpha$ (see Sect. 2) increase the error to $\left(0.54_{-0.04}^{+0.06}\right) \%$.

\subsection{Electrode shift}

The distribution of the electrode shift as a function of the azimuthal angle is presented in Fig. 21 for layer 2. A rather flat behavior is observed. Vertical dashed lines correspond to the boundaries between consecutive modules. With a finer binning no particular increase of the shift is observed at these transitions, even when extending the scale to $1000 \mu \mathrm{m}$. The average of about $146 \mathrm{~m}$ is independent of the layer.

\section{Drift time and velocity measurements}

To quantify the consistency of the drift time measurements, the drift velocity ( $V_{\text {drift }}$ ) is studied more closely. The drift velocity can be extracted from drift time measurements if the local gap values are accurately known (see (1)). Both $w_{\text {gap }}$ and $T_{\text {drift }}$ are designed to be constant for the barrel, but varying with pseudorapidity for the endcap. The variation of the drift time $T_{\text {drift }}$ (see Fig. 22(a)) does not compensate for 


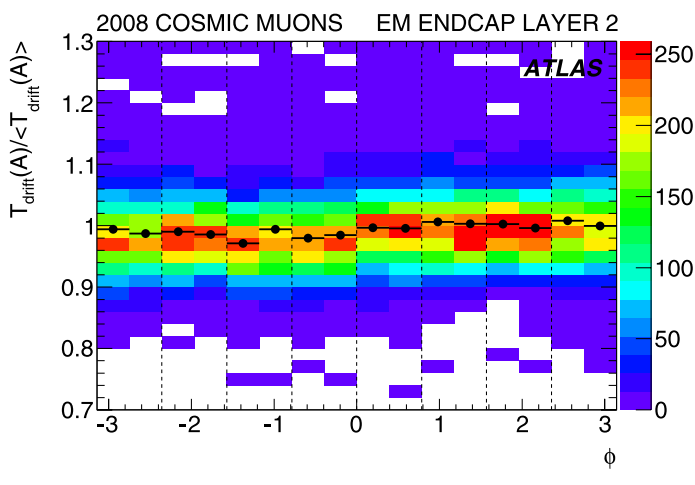

(a) layer 2 of endcap A

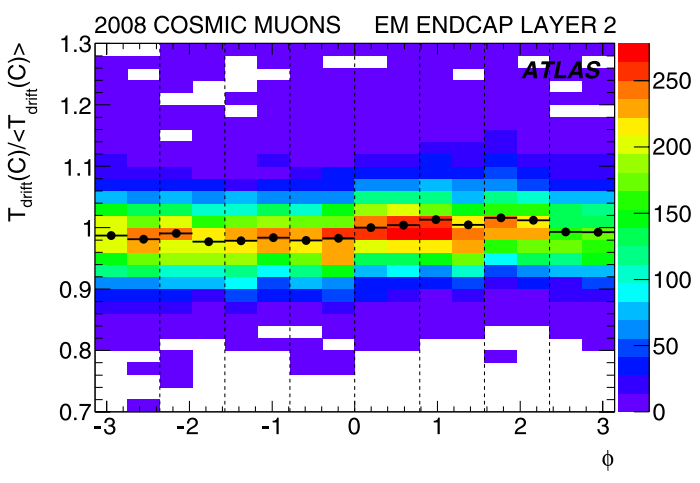

(b) layer 2 of endcap C

Fig. 19 Drift time normalized to the average value versus $\phi$ for layer 2 of the $\eta>0$ (a) and $\eta<0$ (b) endcap wheels. The black dots are the average per $\phi$ bin and the vertical dashed lines show the boundaries between different modules

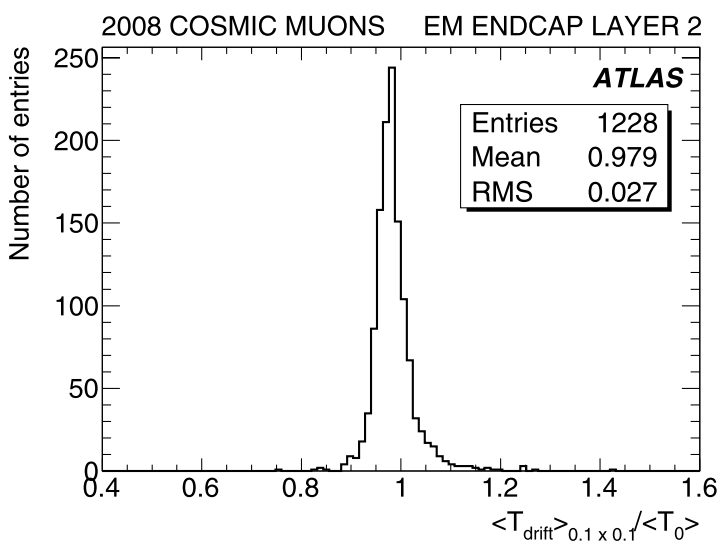

Fig. 20 Drift time uniformity between groups of $4 \times 4$ cells $(\Delta \eta \times \Delta \phi=0.1 \times 0.1)$ for endcap layer 2 . The normalization $\left\langle T_{0}\right\rangle$ is obtained as a fit to the data using a first order polynomial in each HV region to cancel out the influence of the gap variation with $\eta$

the variation of $w_{\text {gap }}$ because $T_{\text {drift }} \sim w_{\text {gap }}^{1+\alpha}$. In addition, the different high voltage regions in the endcap introduce steps in the behavior of the drift velocity as a function of $\eta$.

In order to compare accurately the drift velocities between barrel and endcap and for each calorimeter layer, they are scaled to a reference field of $1 \mathrm{kV} / \mathrm{mm}$ :

$V_{\text {drift }}(1 \mathrm{kV} / \mathrm{mm})=\frac{w_{\text {gap }}}{T_{\text {drift }}}\left(\frac{2000 \mathrm{~V} \cdot w_{\text {gap }}}{H V_{\text {nom }} \cdot 2 \mathrm{~mm}}\right)^{\alpha}$

where $H V_{\text {nom }}$ is the nominal high voltage value, $w_{\text {gap }}$ is taken from the design value and $\alpha$ is the exponent introduced in Sect. 2. Figure 22(a) shows the drift velocity at the same field $1 \mathrm{kV} / \mathrm{mm}$ for layer 2 of the entire calorimeter as a function of $\eta$. As expected, a rather constant behavior is observed over the entire calorimeter. The deviations from a perfect horizontal line is explained by local non-uniformities. Deviations are observed at the transition regions at $\eta=0$ and

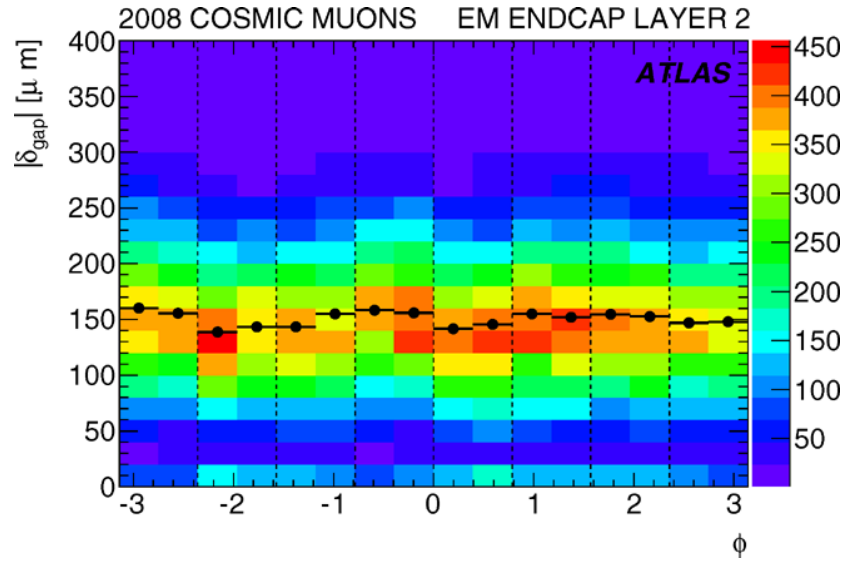

Fig. 21 Electrode shift as function of $\phi$ for layer 2 of the endcap. The black dots are the average per $\phi$ bin and the vertical dashed lines show the boundaries between different modules

$|\eta|=0.8$ and in the crack region between barrel and endcap at $|\eta|=1.4$, where the field is lower.

The temperature in the endcap A $(\eta>0)$ is slightly higher (by about $0.3 \mathrm{~K}$ ) than the temperatures of the barrel $(88.5 \mathrm{~K})$ and endcap $\mathrm{C}(88.4 \mathrm{~K})$. This can explain the larger drift velocity measured in endcap $\mathrm{C}(\eta<0)$ with respect to endcap A, by $\sim 0.6 \%$ (see Fig. 22(b)), the expected difference being approximately $0.5 \%$.

Figure 23 shows the comparison of $V_{\text {drift }}$ for the different layers of the barrel and endcaps. The mean values of the distributions are also quoted. The errors on these means, given the large number of pulses averaged and the random nature of the noise dominating the error on single measurements, are much smaller than the systematic uncertainties (see Sect. 9). According to (16), the uncertainty in the drift velocity depends on uncertainties in both the gap size and the drift time. The former can be extracted from an azimuthal and pseudorapidity uniformity study, giving values smaller or equal to $1 \%$ and $2 \%$ for the barrel and endcap 


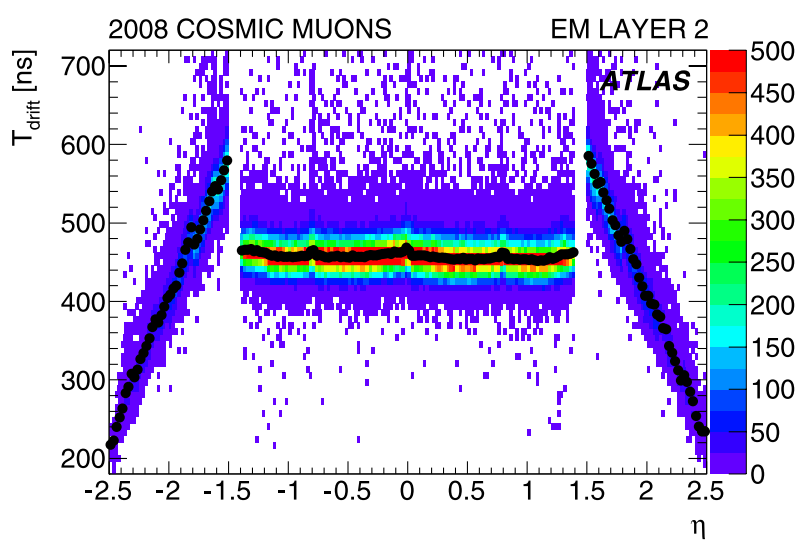

(a) Drift time

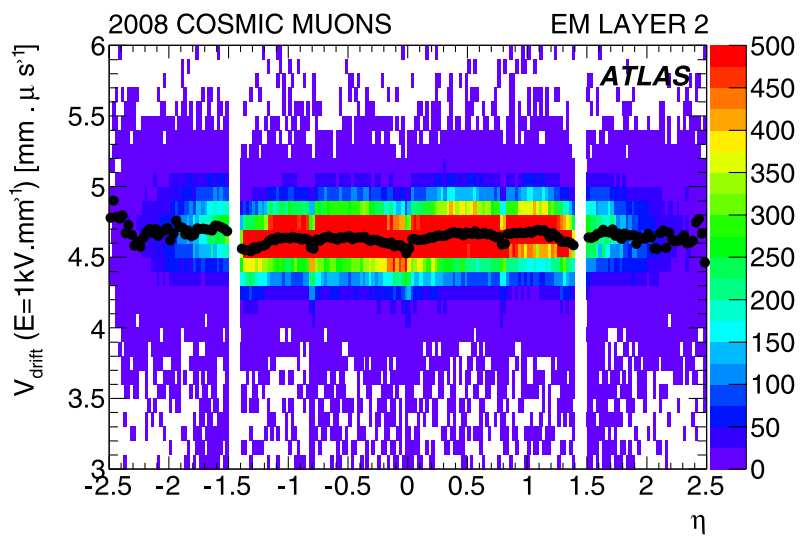

(b) Drift velocity

Fig. 22 (a) Drift time and (b) Drift velocity (at $E=1 \mathrm{kV} / \mathrm{mm}$ ) versus $\eta$ in layer 2. The black dots are the average per $\eta$ bin

respectively. The latter receives contributions from several sources (see Sect. 9). The mean values of the drift velocity for the different layers of the barrel and endcap are given in Table 6. They are all compatible within errors, although the barrel presampler is somewhat below the average.

These results can be compared with the measurements from [11] which give $(4.65 \pm 0.12) \mathrm{mm} / \mu$ s for a LAr tem-

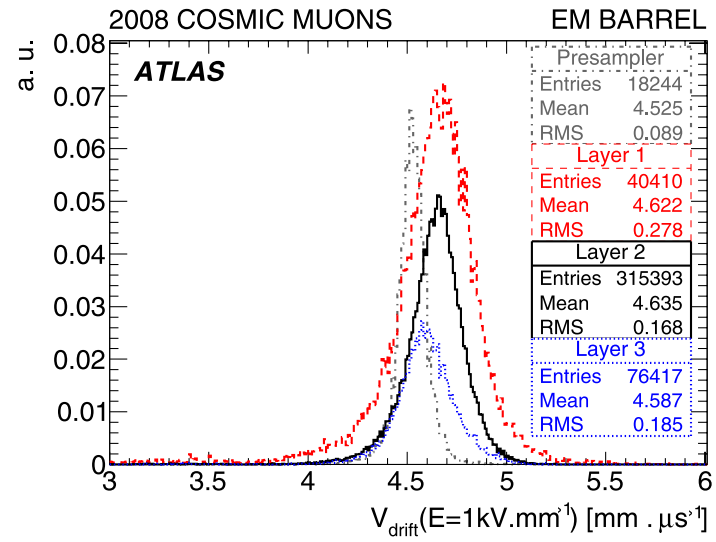

(a) Barrel

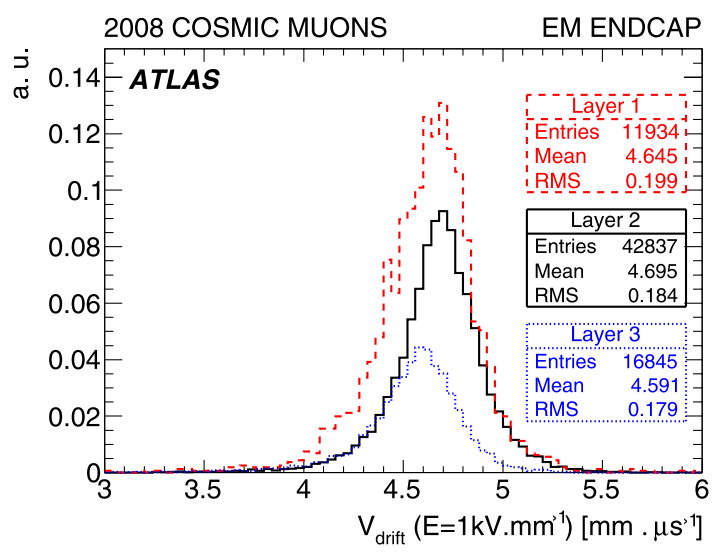

(b) Endcap

Fig. 23 Drift velocity distribution for the barrel (a) and endcap (b)

perature of $88.5 \mathrm{~K}$ and provides good agreement with the present measurement.

\section{Systematic uncertainties}

The different sources of systematic uncertainties affecting the measurement of the drift time which have been studied are discussed below. The resulting systematic uncertainties
Table 6 Drift velocity at $E=1 \mathrm{kV} / \mathrm{mm}$ in the different layers of the calorimeter

\begin{tabular}{|c|c|c|}
\hline & Layer & Drift velocity (in $\mathrm{mm} / \mu \mathrm{s}$ at $1 \mathrm{kV} / \mathrm{mm}$ ) \\
\hline \multirow[t]{4}{*}{ Barrel } & Presampler & $4.52 \pm 0.001$ (stat) ${ }_{-0.07}^{+0.11}$ (syst) \\
\hline & Layer 1 & $4.62 \pm 0.003$ (stat) ${ }_{-0.14}^{+0.06}$ (syst) \\
\hline & Layer 2 & $4.63 \pm 0.002$ (stat) ${ }_{-0.14}^{+0.06}$ (syst) \\
\hline & Layer 3 & $4.59 \pm 0.002$ (stat) ${ }_{-0.14}^{+0.06}($ syst $)$ \\
\hline \multirow[t]{3}{*}{ Endcap } & Layer 1 & $4.65 \pm 0.002(\text { stat })_{-0.14}^{+0.10}($ syst $)$ \\
\hline & Layer 2 & $4.69 \pm 0.001$ (stat) ${ }_{-0.14}^{+0.10}$ (syst) \\
\hline & Layer 3 & $4.59 \pm 0.002$ (stat) ${ }_{-0.14}^{+0.10}$ (syst) \\
\hline
\end{tabular}


on the velocity are given in Table 6, and in Sects. 6.2 and 7.2 for what concerns the uniformity of response.

\subsection{Comparison of the results obtained in the barrel} with the two prediction methods

Two pulse shape prediction methods have been used for the barrel. Their results are compared to give an estimate of the systematic uncertainty on the prediction. For layers 2 and 3, the mean value of the difference between the predicted distributions is $\sim 0.2 \mathrm{~ns}$ and the RMS in the $\eta$ direction is $\sim 1.2 \mathrm{~ns}$ which is of the order of the precision of the measurement for both methods: hence no significant difference is observed for these layers. For layer 1, which also suffers from low statistics, the mean value of the difference ( $1.3 \mathrm{~ns}$ ) (see Sect. 6.1.2) is taken as an estimate of the systematic uncertainty associated with the prediction.

\subsection{Different fit strategies}

In addition to the fit procedure described in this paper, another approach was also followed in layer 2 of the barrel: the cell-based fit. The method consists of fitting simultaneously all the $(N)$ pulses collected in a given cell, using a single value for each of the drift time and the shift parameter, and $N$ global normalization factors and timing adjustments (one of each per pulse). This yields results that are similar but not identical to those obtained from a weighted average of the individual fits with the weight $\left(S_{\max }^{\text {gain }}\right)^{2}$. For instance the average drift time in the case of the cell-based fit is 1.2 ns (i.e. $0.3 \%$ ) lower due to a somewhat reduced effect of pulses with large $T_{\text {drift }}$. With the cell-based method, which has more statistical power for a given fit, the spike at zero visible in Fig. 9 is very much reduced, confirming that it originates from statistical fluctuations of the noise leading to a rising slope around $550 \mathrm{~ns}$ steeper than for a single triangle.

\subsection{Variation of parameters of the cell}

The effect of the uncertainty on the capacitance in layer 2 of the barrel on the FPM determination of the drift time is studied as follows: the capacitance is varied by $\pm 5 \%$ based on measurements, and a new set of the parameters $\tau_{\mathrm{sh}}$ and $Z_{\mathrm{S}}$ (defined in Sect. 3) are recalculated from the FPM calibration fits and used in new fits of the cosmic muon data. A small change in the overall drift time scale is observed, but no significant variation in the drift time dependence on $\eta$. It should nevertheless be noted that when varying the capacitance in either direction, the drift times increased by about 3 ns. As discussed in [14], an increase (decrease) in the value of the capacitance is partially compensated by a smaller (larger) value of the shaper time constant $\tau_{\mathrm{sh}}$, which leads to only minor variations in the pulse shape.
For the RTM method, the estimated uncertainty for the determination of $L C$ and $\tau_{\text {cali }}$ is less than $3 \%$. The $\tau_{\text {cali }}$ uncertainty induces an uncertainty of about $0.5 \%$ on $T_{\text {drift }}$, with an additional contribution of less than $0.1 \%$ coming from the $L C$ uncertainty.

\subsection{Effect of electron attachment}

In the presence of impurities in the LAr medium, drift electrons may attach to the impurities with an associated lifetime $T_{\text {live }}$, and the signal shape is no longer triangular but has the form:

$I(t)=\frac{Q_{0}}{T_{\mathrm{drift}}} e^{-t / T_{\mathrm{live}}}\left(1-\frac{t}{T_{\mathrm{drift}}}\right) \quad$ for $0<t<T_{\mathrm{drift}}$.

Using the Fourier transform of $I(t)$, the pulse shape is derived by convolution of the various factors affecting pulse formation and propagation (see [14] for the general case). The data are then fitted with the additional parameter $T_{\text {live }}$.

Although this new parametrization allows to reduce the size of residuals, the values obtained for $T_{\text {live }}$ have a large dispersion (about $6 \mu$ s for both the average and RMS of the distribution). Another weak point of this description is that the effect is totally absent in the presampler, which is in the same liquid bath as the calorimeter.

A systematic uncertainty of ${ }_{-0}^{+1.5} \%$ in the drift time is conservatively estimated from the difference between the cases of including or not the $T_{\text {live }}$ parameter. The $\eta$ dependence of $T_{\text {drift }}$ remains essentially the same in both cases. The drift velocity remains unchanged in the presampler, but is reduced by 1.5 to $2 \%$ in the other layers, which would make the presampler and the rest of the barrel more compatible.

While this study was made only in the barrel, the estimated systematic uncertainty is also used for the endcap.

\subsection{Variation of the bent triangle contribution}

The amount of energy deposited in the bent sections of the calorimeter is estimated using the simulation. To account for possible differences between data and simulation, a systematic uncertainty related to the estimate of the fraction of signal collected in the bent sections is assessed by varying the contribution of the triangle associated with the bends $f_{\text {bend }}$ by $\pm 20 \%$ based on Table 1 . This test was done in a limited section of layer 2 of the barrel. The resulting systematic variation of the drift time is $\mp 3 \mathrm{~ns}$, as if $T_{\text {drift }}$ were compensating the absence of the bent triangles. It should be noted that the variations of the drift time with the relative amplitude of the third and fourth triangle (see (4) to (7)) are constant throughout the detector; uncertainties on the contribution from bent sections should therefore not affect the estimate of the intrinsic uniformity, except in layer 1 (see Sect. 6.1.2).

The procedure to estimate $T_{\text {bend }}$ and $f_{\text {bend }}$ in the endcap requires that the contributions from the bent and straight 
parts of the accordion can be separated using the local drift time distribution of simulated $10 \mathrm{GeV}$ photon showers. The uncertainty induced by this procedure is propagated to the final $T_{\text {drift }}$ value, leading to a $0.2 \%$ variation that is compatible with the precision of the measurement.

\subsection{Variation of the parameter $\alpha$}

The effect of the uncertainty on the exponent $\alpha$ in the determination of the drift time in the endcap was studied by varying $\alpha$ in the range from 0.30 to 0.39 larger than the range determined in Sect. 2 (0.28 to 0.34). This larger range was initially motivated by a previous measurement of this exponent during the beam test of the endcap prototype using $120 \mathrm{GeV}$ electrons, where a value of 0.39 seemed to describe the data better, however over a larger electrical field range than relevant here. The effect of this difference $(0.30$ to 0.39$)$ on the drift time is approximately $1 \mathrm{~ns}$ or about $0.2 \%$, again at the level of precision of the measurement. The effect on the retained range $(0.28$ to 0.34$)$ would be even smaller. The arithmetic effect of the uncertainty on $\alpha$ on the uniformity was considered in Sects. 6.2 and 7.2.

\subsection{Summary of the systematic uncertainties}

The systematic uncertainties discussed above apply to the drift time measurements and can be translated into drift velocity through (1). The drift velocities for each layer are summarized in Table 6.

Averaging over the presampler and layer 2 (barrel and endcap) values, for which most of the systematics are uncorrelated, gives as the final result for the reference field of $1 \mathrm{kV} / \mathrm{mm}$ and a temperature $T=88.5 \mathrm{~K}$ :

$V_{\text {ref }}=(4.61 \pm 0.07) \mathrm{mm} / \mu \mathrm{s}$.

\section{Direct determination of local gap and drift velocity at operating point}

Taking advantage of the studies presented above, a somewhat more global treatment of the data is presented below, which allows:

- To unify the comparison of the local measured gaps, and their reference value from construction in both the barrel and the endcaps.

- To obtain for the whole calorimeter the values of the drift velocity at the local operating points.

If the drift velocity were to be fully saturated, i.e. independent of the electric field, a measurement of the drift time would trivially give the associated local gap using (1). In the situation analyzed here, the drift velocity depends weakly on the electric field, with a power law already given in Sect. 2 (see (3)).

Using (1) and (3) rewritten below as

$V_{\text {drift }}=V_{\text {ref }} \cdot\left[\frac{H V}{H V_{0}} \cdot \frac{w_{\text {gap } 0}}{w_{\text {gap }}}\right]^{\alpha}$

it is possible to express both the local velocity and the local gap, as functions of the measured $T_{\text {drift }}$ :

$w_{\text {gap }}=\left[A \cdot T_{\text {drift }}\right]^{1 /(1+\alpha)}$,

$V_{\mathrm{drift}}=\frac{A^{1 /(1+\alpha)}}{T_{\mathrm{drift}}^{\alpha /(1+\alpha)}}$

with $A=V_{\text {ref }} \cdot\left[\frac{H V}{H V_{0}}\right]^{\alpha} \cdot w_{\text {gap0 }}^{\alpha}$. The analysis presented below uses: $\alpha=0.3, w_{\text {gap } 0}=2 \mathrm{~mm}, H V_{0}=2 \mathrm{kV}$ and normalizes the drift velocity at $1 \mathrm{kV} / \mathrm{mm}$ to the average $V_{\text {ref }}=$ $4.61 \mathrm{~mm} / \mu \mathrm{s}$, as reported in Sect. 9. The effect of the shift $(x \sim 0.1)$ was estimated to bias the above analysis by less than $0.2 \%$ on the extracted gap value, and is therefore neglected. Data for the endcaps have been corrected for the temperature difference, and rescaled to $88.5 \mathrm{~K}$.

The additional information yielded by this analysis shows directly how the ratio of the measured gap to the designed gap varies as a function of position in the detector. Figure 24 shows the relative difference between the calculated and design values. The average difference is not exactly 0 . This comes from the fact that the average velocity value used for the normalization includes presampler data, while the gap calculation presented in Fig. 24 contains only layer 2.

One can see that the ratio between calculated and design values, spanning a gap range between $1 \mathrm{~mm}$ and $2.5 \mathrm{~mm}$, has an RMS of $0.83 \%$, i.e. typically $16 \mu \mathrm{m}$. In the presampler, the corresponding dispersion is $7 \mu \mathrm{m}$, reflecting a more rigid fixing of the electrodes defining the gaps. In the barrel part one recognizes the systematic effects in the results discussed in Sect. 6.1.1 (see in particular Fig. 10) associated with the

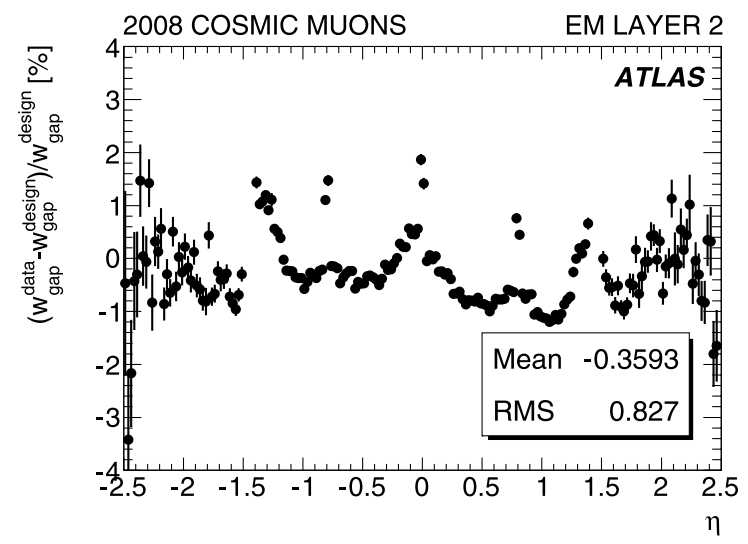

Fig. 24 Relative difference between the design gap values and the values extracted from $T_{\text {drift }}$ measurements 
slight bulging of the absorbers, and the "transition regions" at $\eta=0, \pm 0.8$ and \pm 1.4 . Strictly speaking these transitions areas, for which additional effects enter into play, should be corrected for in the calculation of the RMS. In the endcaps the statistical power is unfortunately lower giving rise to larger fluctuations, but no significant trend is observed.

Figure 25 shows the drift velocity obtained using (21) as a function of pseudorapidity and the same normalization as above. As opposed to Fig. 22, which gave the velocity at a reference field of $1 \mathrm{kV} / \mathrm{mm}$, Fig. 25 shows the drift velocity at the local operating field, which is directly related to the peak current (see Sect. 2) associated with an energy deposition.

In the barrel region, the drift velocity is essentially flat, with a slight modulation reflecting the variation of the absorber thickness with pseudorapidity. Taking the average value of the drift velocity in sectors of $\Delta \eta \times \Delta \phi=0.1 \times 0.1$, as was done in Sect. 6.2 for $T_{\text {drift }}$, one obtains a distribution with an RMS of $0.29 \%$ exactly equal to what was derived in Sect. 6.2 from the RMS of the $T_{\text {drift }}$ distribution, showing the expected consistency of the analyses using $T_{\text {drift }}$ or $V_{\text {drift }}$.

In the endcap region, one observes the 6 sawteeth on each side resulting from the finite granularity of the $\mathrm{HV}$ distribution (see Fig. 3). Corrections are made in the energy reconstruction to normalize the response of each strip in pseudorapidity to the response of the strip in the center of the $\mathrm{HV}$ sector, using the power law dependence. Beside these modulations, one observes that:

- The average velocity in the endcaps is smaller than in the barrel. In the energy reconstruction this is accounted for by correction factors (which also take into account the fact that the lead thicknesses are different) determined from test beam and implemented in the detailed Monte Carlo simulation of the full ATLAS detector.

- The measured velocity averaged over an HV sector somewhat diminishes with increasing pseudorapidity. This effect goes in the same direction (lowering the response)

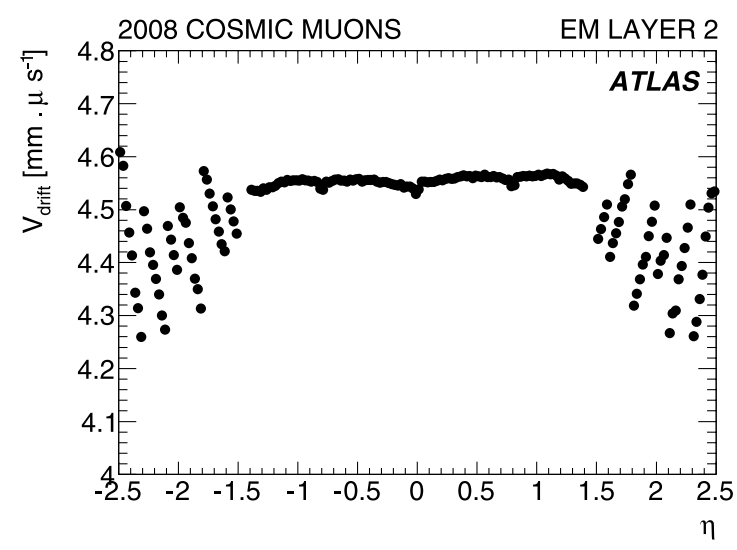

Fig. 25 Drift velocity versus $\eta$ in the layer 2 at the operating point extracted from $T_{\text {drift }}$ measurements as the reduced contribution of liquid argon to showering/conversion effects at large pseudorapidities (small gaps). Both effects are qualitatively counterbalanced by the fact that the relative contribution of bends as compared to flat parts is lower at high pseudorapidity, resulting in an increased response. As already mentioned, detailed Monte Carlo simulations normalized with test beam scans were used to determine the HV values optimizing the uniformity of response of the endcaps. This will be cross checked when enough $Z^{0} \rightarrow e^{+} e^{-}$decays become available.

\section{Conclusions}

We have shown in this paper that sufficient amounts of ionization data $(\sim 0.5$ million pulses of energy larger than $\sim 1 \mathrm{GeV}$ ) can be used for a precision measurement of the average electron drift time in each cell of the highly granular LAr electromagnetic calorimeter of ATLAS that has been readout with fast electronics, in the current mode. In this regime, the recorded energy is directly proportional to the drift velocity of ionization electrons, which is readily obtained from the drift time measurement. Furthermore, the drift velocity and thus the recorded energy are $\sim 4$ times less sensitive to gap variations than the drift time.

Taking advantage of these facts, we derived an estimate of the calorimeter non-uniformity of response due to gap size variations, of $\left(0.29_{-0.04}^{+0.05}\right)$ and $\left(0.54_{-0.04}^{+0.06}\right) \%$ respectively for the barrel and the endcaps. The other main contribution to the intrinsic non-uniformity of the calorimeter is the dispersion of the thickness of the lead absorbers which contributes $0.18 \%$ for both barrel and endcaps $[2,3]$.

The drift time is also an input needed in order to reconstruct the signal amplitude by optimal filtering. An examination of the tails of the drift time distributions singles out "transition areas" of the calorimeter, in both azimuthal or pseudorapidity angle, where the electrical field is lower than average due to "edge effects". Some modulations in the third layer of the barrel have also been observed.

The analysis method used to derive the drift time provides as another parameter the average absolute value of the amount the electrodes are off center between their two neighboring absorbers. The values obtained are around $146 \mu \mathrm{m}$ for both barrel and endcap accordion layers, and are substantially smaller for the presampler $(66.5 \mu \mathrm{m})$ as expected from its design.

The drift velocity, rescaled to a field of $1 \mathrm{kV} / \mathrm{mm}$, is obtained from the drift time measurements leading to an average of $(4.61 \pm 0.07) \mathrm{mm} / \mu \mathrm{s}$. This value is compatible with previously published measurements at the same operating temperature of $88.5 \mathrm{~K}$.

The measurements presented in this paper illustrate the accuracy achieved with this method even using cosmic 
muon data, thus demonstrating that it can be used to correct for the measured gap variations in order to eventually reduce the constant term of the energy resolution, especially if the measurements are repeated with collision data. It is therefore important, in the quest to improve the energy resolution constant term, that in the future these measurements be done with LHC collision data.

Acknowledgements We are greatly indebted to all CERN's departments and to the LHC project for their immense efforts not only in building the LHC, but also for their direct contributions to the construction and installation of the ATLAS detector and its infrastructure. We acknowledge equally warmly all our technical colleagues in the collaborating institutions without whom the ATLAS detector could not have been built. Furthermore we are grateful to all the funding agencies which supported generously the construction and the commissioning of the ATLAS detector and also provided the computing infrastructure.

The ATLAS detector design and construction has taken about fifteen years, and our thoughts are with all our colleagues who sadly could not see its final realization.

We acknowledge the support of ANPCyT, Argentina; Yerevan Physics Institute, Armenia; ARC and DEST, Australia; Bundesministerium für Wissenschaft und Forschung, Austria; National Academy of Sciences of Azerbaijan; State Committee on Science \& Technologies of the Republic of Belarus; CNPq and FINEP, Brazil; NSERC, NRC, and CFI, Canada; CERN; NSFC, China; Ministry of Education, Youth and Sports of the Czech Republic, Ministry of Industry and Trade of the Czech Republic, and Committee for Collaboration of the Czech Republic with CERN; Danish Natural Science Research Council; European Commission, through the ARTEMIS Research Training Network; IN2P3-CNRS and Dapnia-CEA, France; Georgian Academy of Sciences; BMBF, DESY, DFG and MPG, Germany; Ministry of Education and Religion, through the EPEAEK program PYTHAGORAS II and GSRT, Greece; ISF, MINERVA, GIF, DIP, and Benoziyo Center, Israel; INFN, Italy; MEXT, Japan; CNRST, Morocco; FOM and NWO, Netherlands; The Research Council of Norway; Ministry of Science and Higher Education, Poland; GRICES and FCT, Portugal; Ministry of Education and Research, Romania; Ministry of Education and Science of the Russian Federation, Russian Federal Agency of Science and Innovations, and Russian Federal Agency of Atomic Energy; JINR; Ministry of Science, Serbia; Department of International Science and Technology Cooperation, Ministry of Education of the Slovak Republic; Slovenian Research Agency, Ministry of Higher Education, Science and Technology, Slovenia; Ministerio de Educación y Ciencia, Spain; The Swedish Research Council, The Knut and Alice Wallenberg Foundation, Sweden; State Secretariat for Education and Science, Swiss National Science Foundation, and Cantons of Bern and Geneva, Switzerland; National Science Council, Taiwan; TAEK, Turkey; The Science and Technology Facilities Council and The Leverhulme Trust, United Kingdom; DOE and NSF, United States of America.
Open Access This article is distributed under the terms of the Creative Commons Attribution Noncommercial License which permits any noncommercial use, distribution, and reproduction in any medium, provided the original author(s) and source are credited.

\section{References}

1. ATLAS Collaboration, The ATLAS experiment at the CERN large hadron collider. JINST 3, S08003 (2008)

2. B. Aubert et al., Construction, assembly and tests of the ATLAS electromagnetic barrel calorimeter. Nucl. Instrum. Methods A 558, 388 (2006)

3. M. Aleksa et al., Construction, assembly and tests of the ATLAS electromagnetic end-cap calorimeter. JINST 3, P06002 (2008)

4. M.L. Andrieux et al., Construction and test of the first two sectors of the ATLAS liquid argon presampler. Nucl. Instrum. Methods A 479, 316 (2002)

5. ATLAS Collaboration, Readiness of the ATLAS liquid argon calorimeter for LHC collisions, submitted for publication in EPJC. arXiv:0912.2642v2

6. ATLAS Collaboration, ATLAS liquid argon calorimeter. Technical design report, ATLAS-TDR-002, CERN-LHCC-96-41

7. N.J. Buchanan et al., ATLAS liquid argon calorimeter front end electronics. JINST 3, P02010 (2008)

8. A. Bazan et al., ATLAS liquid argon calorimeter back end electronics (RODs). JINST 2, P06002 (2007)

9. L.S. Miller et al., Charge transport in solid and liquid $\mathrm{Ar}, \mathrm{Kr}$ and Xe. Phys. Rev. 166, 871-878 (1968)

10. K. Yoshino et al., Effect of molecular solutes on the electron drift velocity in liquid Ar, Kr, and Xe. Phys. Rev. A 14, 438-444 (1976)

11. W. Walkowiak, Drift velocity of free electrons in liquid argon. Nucl. Instrum. Methods A 449, 288-294 (2000)

12. W.E. Cleland, E.G. Stern, Signal processing considerations for liquid ionization calorimeter in a high rate environment. Nucl. Instrum. Methods A 338, 467 (1994)

13. D. Banfi, M. Delmastro, M. Fanti, Cell response equalization of the ATLAS electromagnetic calorimeter without the direct knowledge of the ionization signals. JINST 1, P08001 (2006)

14. C. Collard, D. Fournier, S. Henrot-Versillé, L. Serin, Prediction of signal amplitude and shape for the ATLAS electromagnetic calorimeter. ATL-LARG-PUB-2007-010

15. M. Frigo, S.G. Johnson, The design and implementation of FFTW3. Proc. IEEE 93 2, 216-231 (2005)

16. J. Colas et al., Electronics calibration board for the ATLAS liquid argon calorimeters. Nucl. Instrum. Methods A 593, 269 (2008)

17. S. Baffioni et al., Electrical measurements on the ATLAS electromagnetic barrel calorimeter. ATL-LARG-PUB-2007-005

18. F. James, M. Roos, Minuit: a system for function minimization and analysis of the parameter errors and correlations. Comput. Phys. Commun. 10, 343-367 (1975) 